\title{
OPTIMAL MOTION PROGRAMMING \\ OF ROBOT MANIPULATORS
}

\author{
By \\ DAN JOE SCHMITT \\ Bachelor of Science in Mechanical Engineering \\ Oklahoma State University \\ Stillwater, Oklahoma \\ 1981
}

Submitted to the Faculty of the Graduate College of the Oklahoma State University

in partial fulfillment of the requirements for the Degree of

MASTER OF SCIENCE

July, 1983 
OPTIMAL MOTION PROGRAMMING

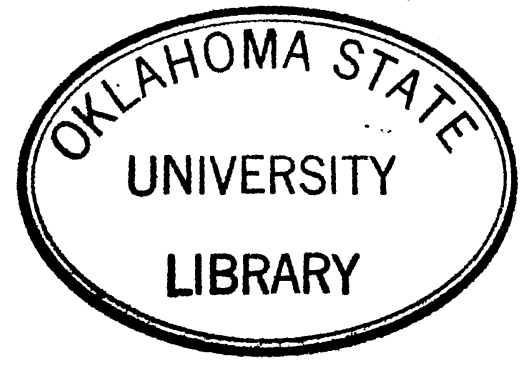

OF ROBOT MANIPULATORS

Thes is Approved:

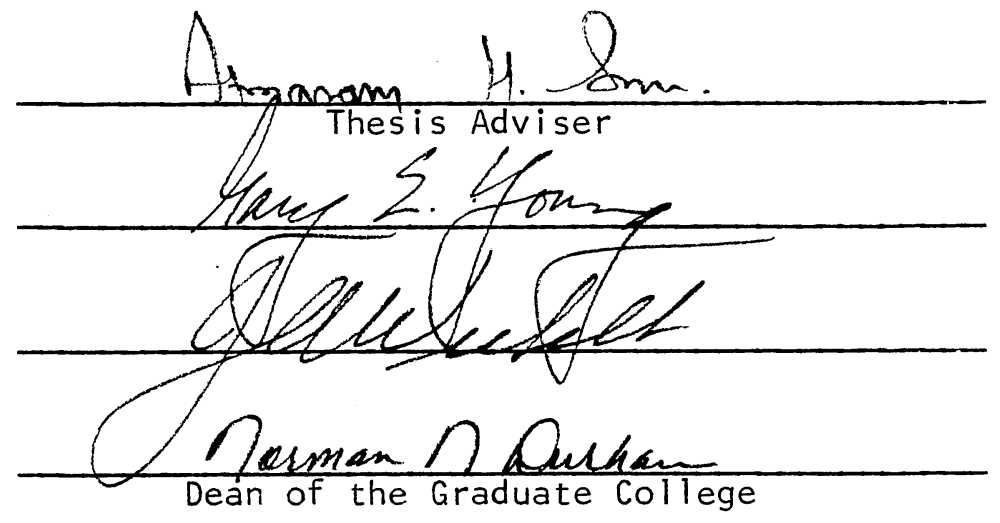


PREFACE

This study is concerned with optimal motion programming of robot manipulators. The primary objective is to develop a procedure to determine optimal motion programs for any robot configuration. The resulting motion programs are optimal with respect to the torques at the robot's joints. A computer algorithm is utilized to carry out the optimization process.

The author wishes to express his appreciation to his major adviser, Dr. A. H. Soni, for his guidance in this research effort. Also, a special thanks to Dr. V. J. Srinivasan for his technical guidance, and for meeting with the author on a regular basis. Also, appreciation is expressed to the other committee members, Dr. J. J. Allen and Dr. G. E. Young, for their assistance.

A note of thanks if given to Ms. Charlene Fries and Ms. Jama Leitzke for their work in preparing the final document. The author would also like to thank the School of Mechanical and Aerospace Engineering for providing a teaching assistantship during his graduate studies. 
TABLE OF CONTENTS

Chapter

Page

I. INTRODUCTION . . . . . . . . . . . . . . . . . . . . 1

1.1 Literature Review . . . . . . . . . . . 1

1.2 Proposed Research ............... 9

1.3 Organization ............. . . 10

11. THEORY DEVELOPMENT . . . . . . . . . . . . . . 12

2.1 Definition of the Problem . . . . . . . . 12

2.2 Formulation of the Problem ........... 14

2.3 Method of Solution.............. 16

2.4 Choice of Approximating Functions . . . . . . . 19

2.5 Computational Procedure .......... 24

111. APPLICATION OF THE THEORY TO A 2-R ROBOT......... . 30

3.1 Scope of Chapter ............... 30

3.2 Problem to be Solved............. 30

3.3 Determination of Required Number of Terms . . . 36

3.4 Comparison of Polynomial and Trigonometric Approximating Functions . . . . . . . . . . 44

3.5 Determination of Optimal Traveling Time, T. . . 48

3.6 Comparison of Objective Functions . . . . . . 52

3.7 Comparison of Optimal and Standard Solutions ................ . 69

3.8 Optimal Motion Programming for New Workstations............. 80

IV. APPLICATION OF THE THEORY TO A 3-R ROBOT . . . . . . . . . 97

4.1 Scope of the Chapter . . . . . . . . . . . . 97

4.2 Problem to be Solved . . . . . . . . . . . . . . 97

4.3 Determination of an Optimal Motion Program . . . 102

4.4 Comparison of Optimal and Standard Solutions . . 110

V. SUMMARY AND CONCLUSIONS ............... 125

5.1 Summary of Results and Comments . . . . . . . 125

5.2 Procedure for Solving Optimal Motion

Programming Problems........... . 127

5.3 Extension of the Work . . . . . . . . . . 128

REFERENCES .......................... . 129 
APPENDIX . . . . . . . . . . . . . . . . . . 132 
Table

1. Comparison of Increasing Term Trigonometric Approximations at $\mathrm{T}=0.45$ Seconds . . . . . . . . 37

11. Comparison of Polynomial and Trigonometric Approximating Functions at $T=0.45$ Seconds ... . . . . . . . 46

111. Determination of Optimal Traveling Time, T . . . . . 51

IV. Comparison of objective Functions . .......... . 56

V. Determination of Optimal Time for Standard Solution . . . 81

VI. Determination of Optimal Time for New Workstations . . . 82

VII. Comparison of Increasing Term Trigonometric Approximations at $\mathrm{T}=0.69$ Seconds .......... 85

VIII. Determination of Optimal Traveling Time for the $3-R$ Robot .................... 104

Ix. Comparison of Increasing Term Trigonometric Approximations at $\mathrm{T}=0.63$ Seconds . . . . . . . . 105 
LIST OF FIGURES

Figure Page

1. General Robot Configuration . . . . . . . . . . 13

2. Polynomial Functions of Increasing Order . . . . . . . 23

3. Trigonometric Functions of Increasing Harmonics . . . . . 25

4. Simplified Flow Chart of the SUMT Algorithm . . . . . . . 29

5. Two Axis Robot Task Description . . . . . . . . . . 31

6. Free-Body Diagram of Two-Axis Robot ............ 33

7. Comparison of Increasing Term Trigonometric Approximations of Displacements at Joint 1. . . . . . . . . . . . 39

8. Comparison of Increasing Term Trigonometric Approximations of Displacements at Joint 2........... . . 40

9. Comparison of Torques at Joint 1 for Increasing Term Trigonometric Approximations ... . . . . . . . 41

10. Comparison of Torques at Joint 2 for Increasing Term Trigonometric Approximations . . . . . . . . . 42

11. Comparison of End Effector Paths for Increasing Term Trigonometric Approximations . . . . . . . . . . 43

12. Comparison of Displacements at Joint 1 for $T=1.0$ Seconds.................. . . 45

13. Comparison of Displacements at Joint 2 for $T=1.0$ Seconds................... . . 46

14. Comparison of Trigonometric and Polynomial Approximations of Displacements at Joint 1... . . . . . . . . 49

15. Comparison of Trigonometric and Polynomial Approximations of Displacements at Joint 2.......... . 50

16. End Effector Path at $T=.45$ Seconds . . . . . . . . . . 53

17. End Effector Path at $T=1.46$ Seconds . . . . . . . . . . . 54 
18. Comparison of Displacements at Joint 1 for Various Objective Functions............... 58

19. Comparison of Displacements at Joint 2 for Various Objective Functions . . . . . . . . . . . . . . .

20. Comparison of Torques at Joint 1 for Various Objective Functions . . . . . . . . . . . . . 6 60

21. Comparison of Torques at Joint 2 for Various Objective Functions.....................

22. Comparison of End Effector Paths for Various Objective Functions.....................

23. Torque at Joint 1 for $T=1.457$ Seconds . . . . . . . . . . 64

24. Torque at Joint 2 for $T=1.457$ Seconds . . . . . . . . 65

25. Orientation of Link 2 for $T=1.457$ Seconds . . . . . . . 66

26. Torques at Joint 1 for Objective Functions 1 and 3 . . . . 67

27. Torques at Joint 2 for Objective Functions 1 and 3 .... 63

28. End Effector Paths for Objective Functions 1 and 3 . . . . 70

29. Standard End Effector Path and Motion Program . . . . . . . 71

30. Comparison of Joint 1 Displacements for Optimal and Standard Solutions ............ . 75

31. Comparison of Joint 2 Displacements for Optimal and Standard Solutions ............. . 76

32. Comparison of Joint 1 Torques for Optimal and Standard Solutions

33. Comparison of Joint 2 Torques for Optimal and Standard Solutions :. . . . . . . . . . . . . . 78

34. Comparison of End Effector Paths for Optimal and Standard Solutions . . . . . . . . . . . 79

35. Second Task Description ............... 82

36. Joint 1 Torques for Objective Functions 1 and 3 . . . . . 86

37. Joint 2 Torques for Objective Functions 1 and 3 . . . . 87

38. Comparison of Displacements at Joint 1 for Optimal and Standard Solutions . . . . . . . . . . . . . 
Figure

Page

39. Comparison of Displacements at Joint 2 for Optimal and Standard Solutions ............. . 99 90

40. Comparison of Torques at Joint 1 for Optimal and Standard Solutions . . . . . . . . . . . . . . 91

41. Comparison of Torques at Joint 2 for Optimal and Standard Solutions . . . . . . . . . . . . 92

42. Comparison of End Effector Paths for Optimal and Standard Solutions . . . . . . . . . . . . . 93

43. Torques at Joint 1 for Different Workspace Locations . . . 94

44. Torques at Joint 2 for Different Workspace Locations . . . 95

45. Three-Axis Robot .................. . . 98

46. Free-Body Diagram of Three-Axis Robot . . . . . . . . . 100

47. Torques at Joint 1 for Objective Functions 1 and 3 . . . . 107

48. Torques at Joint 2 for Objective Functions 1 and 3 . . . 108

49. Torques at Joint 3 for Objective Functions 1 and 3 . . . . 109

50. End Effector Paths in Vertical Plane for Objective Functions 1 and 3 ................ 111

51. End Effector Paths in Horizontal Plane for Objective Functions 1 and 3 . . . . . . . . . . . . . 112

52. Comparison of Displacements at Joint 1 for Optimal and Standard Solutions . . . . . . . . . . . . . 116

53. Comparison of Displacements at Joint 2 for Optimal and Standard Solutions . . . . . . . . . . . . 117

54. Comparison of Displacements at Joint 3 for Optimal and Standard Solutions . . . . . . . . . . . . 118

55. Comparison of Torques at Joint 1 for Optimal and Standard Solutions ... . . . . . . . . . . . 119

56. Comparison of Torques at Joint 2 for Optimal and Standard Solutions . . . . . . . . . . . . 120

57. Comparison of Torques at Joint 3 for Optimal and Standard Solutions . . . . . . . . . . . . . 121

58. Comparison of End Effector Paths in Vertical Plane for Optimal and Standard Solutions . . . . . . . . . . 
59. Comparison of End Effector Paths in Horizontal Plane for Optimal and Standard Solutions . . . . . . . . 124 


\section{LIST OF SYMBOLS}

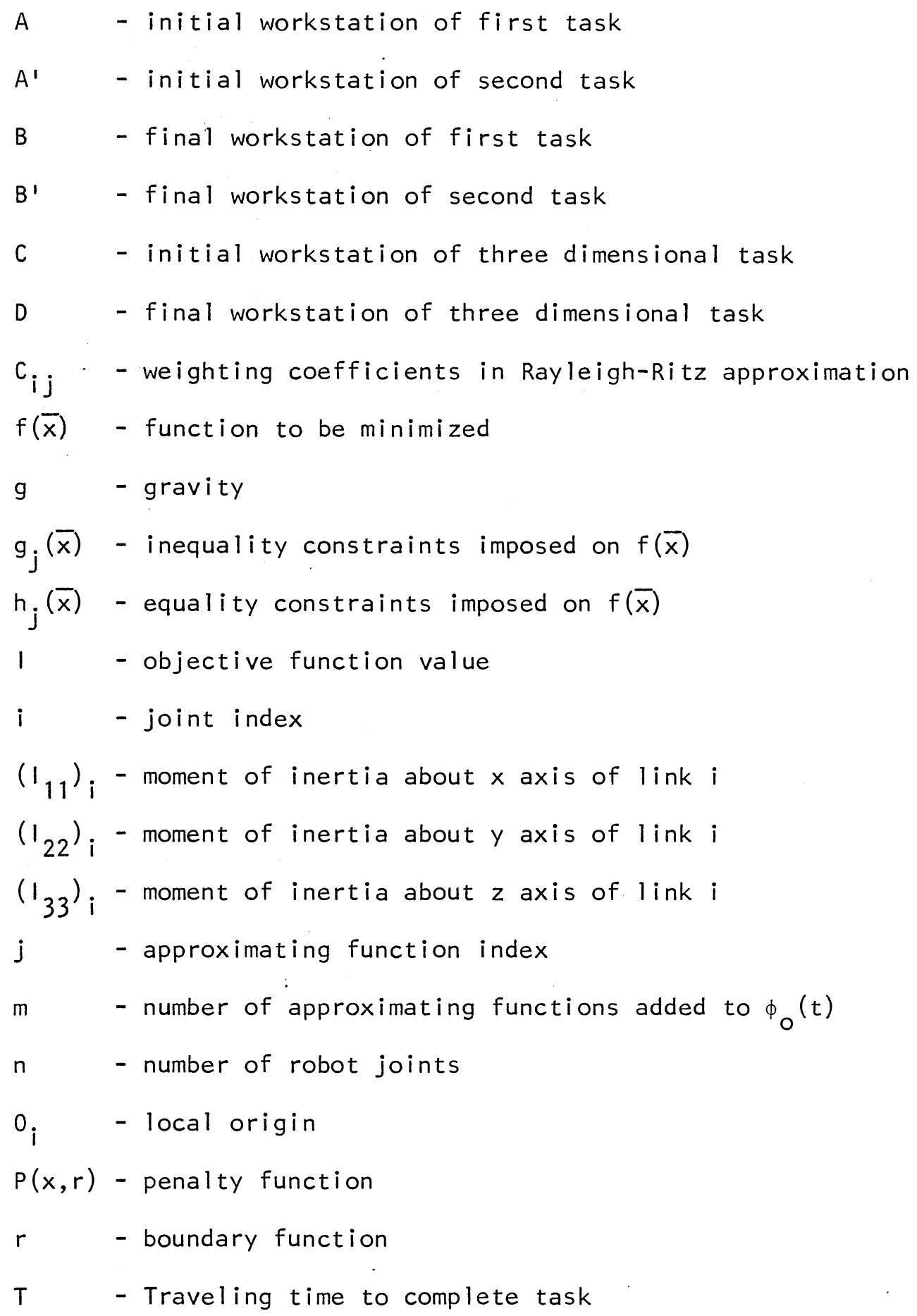




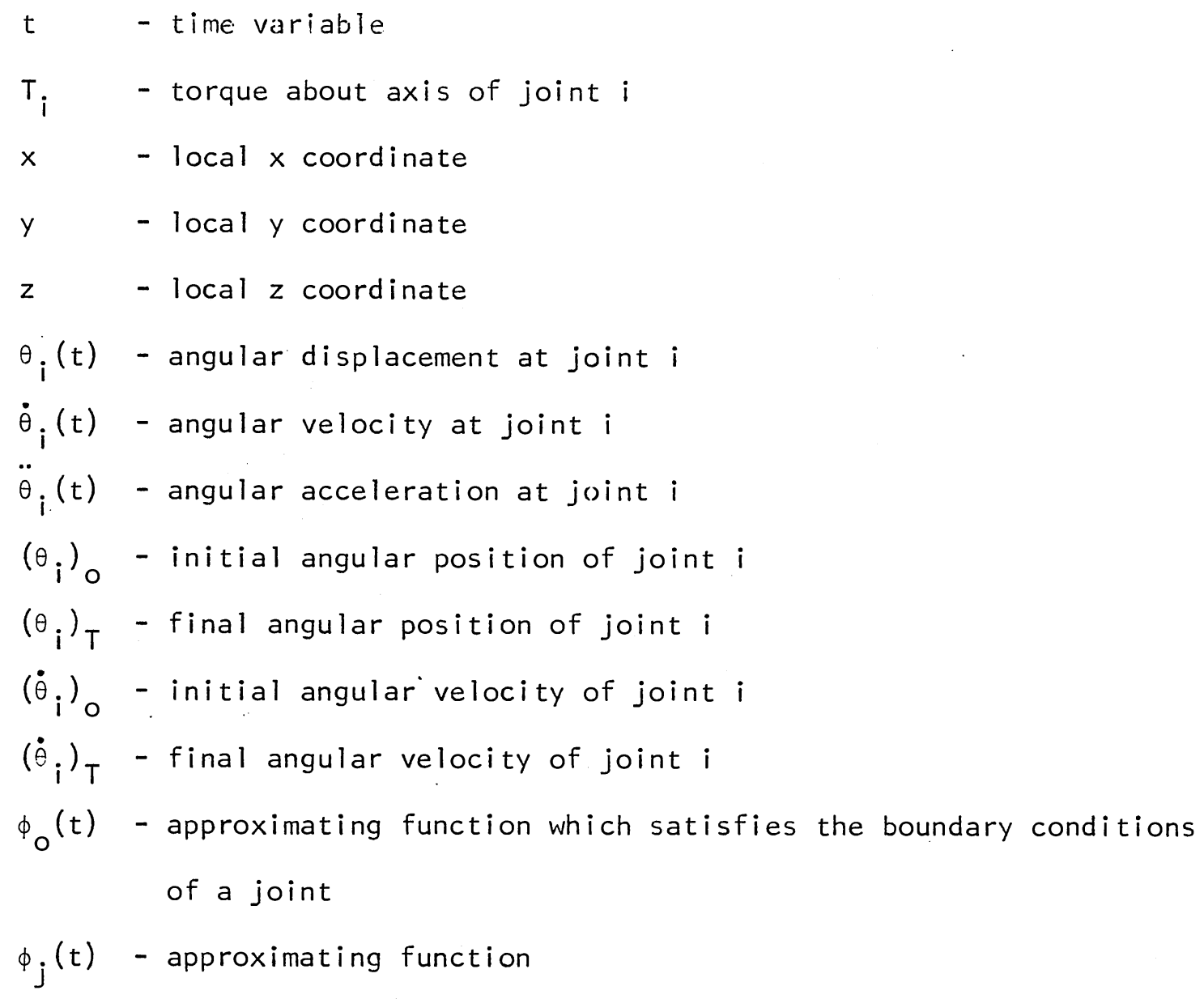


CHAPTER I

INTRODUCTION

\subsection{Literature Review}

Robotics has recently become a major concern within the industry of the United States. The real thrust is on so that the United States can continue to be a tough competitor in the world industrial market. In an industrial interpretation, a robot manipulator is a mechanical - electrical device consisting of a series of links connected by various forms of actuators, which is used to assemble products, move parts, paint, etc. The real advantage found in the use of robots is that they are controlled by an electronic brain in the form of a computer. Thus, the robot is a flexible device that can be reprogrammed to perform a wide variety of tasks.

Robots have been in development and application for several decades. The first robots consisted of open-loop control. A routine was taught to the robot, and it was blindly replayed with hopes that all other pieces the robot had to interact with would be properly located. This approach works, and is still applied today. However, it is not without its difficulties since it is an open-loop device. The present generation of robots is more sophisticated in that they have closed-loop control. Feed back sensors have been added to the end effector, robot joints and at various locations within the workspace. In this way the robot can determine its location at any time, it can determine the location of parts, 
etc. Vision is also being given to the robot so that it can look out into the work environment and make some interpretations about the task to be performed.

The field of robotics requires input from many disciplines within the field of engineering. Mechanical Engineers are involved with the kinematic, dynamic, hydraulic and control aspect of robots. Elecțrical Engineers contribute the electronics to control the entire process, develop control algorithms, and contribute some of the sensor systems to provide feedback for the robot. Industrial Engineers are concerned with integrating the robot into industrial application and developing the work environment for specific robots. The coordinated efforts of all is a. necessity.

One important class of problems in the application of robots is that of programming the robot to perform the desired task. This involves "teaching" the robot the path through which it should move in order to meet the desired objective. Work began in this area in the late 1960's. Pieper [1] and Whitney [2] did work independantly in 1969 to develop rate control algorithms. These algorithms were advantageous to the operator in that they let the operator move the robot arm with external controls, and make these movements in terms of a coordinate system which was easily visible to the operator (i.e., coordinate system attached to the end effector). This was a great improvement over the previously used method of providing motion by controlling the individual joints of the robot. These algorithms took care of transforming the motions indicated by the operator into the separate joint motions necessary to perform the desired movement. Pieper used heuristic control algorithms. Whitney showed that the problem was linear in nature. Whitney [3] also extended this work 
to provide for other criteria in determining the desired joint motions. He provided the technique where some of the joint motions are predefined, and it is desired to minimize some other criteria (i.e., traveling time) over the trajectory. Waldron [4] has recently extended the work of developing rate control algorithms. In his paper, he demonstrates a more efficient formulation of the algorithms based on the geometric characteristics of manipulators as linkages.

In the late 1970's, several people approached the problem of fitting a smooth motion to a path described by a series of connected, straight line segments. Such situations can arise when the desired end effector motion is preplanned before actual execution. Also, in some robot control algorithms, the end effector motion is taught to the robot as a set of discrete points, and it is up to the robot to fit a smooth trajectory to these points in order to provide the end effector motion. Paul [5], [6], [7] looked at this problem in several of his works. Paul states that the basic philosophy behind straight line motion is:

1. No torques are exerted on any load that the hand is carrying. 2. Linear inertial forces are easily calculated.

3. The inertial load is reduced because the hand is brought closer to the base of the manipulator during the motion.

4. The results of such a motion are easily evaluated by "looking along the path" for possible collisions with obstacles. Thus, the theory developed provides for the end effector to move along the straight line segments, but to have a smooth transition between the segments. Paul's earlier works are involved with developing the mathematics for the resulting trajectories. He later developed an algori.thm 
to provide control for the robot. The basic features of the algorithm are:

1. Decision when transitions should be initiated between straight line segments.

2. Generation of setpoints and solution of joint coordinates.

3. Interpolation between setpoints to drive the manipulator.

Luh and Walker [8] have done work on a similar problem. They posed the problem of moving along a series of connected, straight line segments as a linear programming problem. The objective was to determine the joint motions which minimize the traveling time along the resulting path. The inequality constraints imposed were in the allowable positional error in making the curvilinear transitions between the segments, as well as constraints on accelerations and velocities. In 1981, Luh and Lin [9] extended this work by posing the problem as a nonlinear programming problem because of the involvement of a large number of nonlinear, inequality constraints. A "Direct Approximate Programming Algorithm (DAPA)" was developed which took into account some of the special features of the problem. The algorithm was shown to converge to the optimal path.

Taylor [10] has also approached the problem of producing straight line motion between user specified points. He presents two approaches to the problem in his work. The first is a refinement of Paul's work in that better interpolation functions are developed with respect to computational cost and improved motion characteristics. The second method developed involves precomputing intermediate points along the desired path and then interpolating between these points to provide the joint motions for path execution. The precomputed points are spaced such that the deviation from straight line motion during interpolation is held to 
a minimum. Taylor shows that this method provides for a faster algorithm and handles special cases of the robot configuration much more easily. In 1979, Milenkovic [11] improved the concept of fitting a path to a series of user defined points. One problem he saw in fitting straight lines to a series of user defined points was that when the smooth transition was made between the segments, the points were missed by some amount of error. Milenkovic was more interested in end effector motions which would accurately track specific surface contours described by a series of points. He developed higher order interpolation schemes to fit paths which pass directly through the points. This scheme was advantageous in that fewer number of points were needed to describe and track a contour with a certain.accuracy than that for straight line segments. The procedure developed can be programmed for execution in real time.

The next major concern was that of determining how to control the joints of the robot to move along predefined paths (not necessarily straight line). Anderson and Paul [12] developed a control scheme where the equivalent relative position of all the manipulator joints is the same throughout the motion. This technique uses microprocessor based software to determine the slowest joint during a particular motion segment, and controls its motion to drive it at its maximum rate. The motion of the other joints is determined such that the prescribed path is executed with minimal error.

Luh, Walker and Paul [13] considered the path tracking problem in terms of resolved acceleration control. A closed-loop control scheme was developed which first computes joint accelerations from measured velocities and displacements, and uses these values to compute input forces and torques in order to track the desired path. Consideration of 
the actual computation time is given so that the algorithm can provide on-line control. This team extended their work to consider the tracking problem when the end effector load is not known or is variable [14]. This causes variations in joint motions such that the desired path is not followed. This work presents a computational scheme to compute the highly nonlinear dynamic characteristics at frequent intervals within the manipulator motion. The result is the joint forces and torques necessary for the robot to track the desired path. A similar work was carried out by Orlandea and Berenyi [15]. They use the ADAMS program (Automatic Dynamic Analysis of Mechanical Systems) to determine the forces and torques at the robot joints in order to move along the predefined path. A major concern in determining the joint motions to move along a predefined path is moving in an optimal fashion along the path. Vukobratovic and Kircanski [16] developed a simplified dynamic model to determine a manipulator motion which provides optimal velocity distribution along a prescribed path. The resulting motion is optimal with respect to energy consumption. Because of the highly nonlinear nature of this problem, they found that direct application of optimal control theory was difficult. The approach used was that of dynamic programming. This approach solves the problem by finding optimal motions along small segments of the path considered as individual elements. This team later extended their work by developing a complete model of the system which took into account models of the manipulator as well as the actuators [17]. The techniques developed here are for off-line programming.

All of the work previously mentioned involved determining the robot manipulator motion along predefined paths or along paths fit to prescribed points. In some cases the motion was optimized along these paths with 
respect to various criteria (i.e., traveling time, energy, etc.). However, in many cases it is desirable to determine the optimal motion of a robot manipulator when only the initial and final position of the robot are known. Several approaches have been developed. Perez [18] has utilized heuristic methods to determine paths which are minimum with respect to traveling time. The major objective in his work is to provide for obstacle avoidance in moving between two positions. However, he has used an optimal time parameter in order to choose optimal obstacle free paths. Mujtaba [19] has looked at the problem of determining the joint motions when only initial and final positions of the robot are known. He compared several different trajectories which could be used to provide the joint motions. These included a cosine function, a quintic function, and the sum of a sine function and a linear trajectory. The resulting joint motions were determined by fitting the coefficients of these functions to satisfy the boundary conditions of initial and final position, zero initial and final velocity, and an initial and final acceleration that is either zero or a maximum absolute value. Mujtaba has found that the trajectory parameters can be chosen to approximate a bang-bang trajectory. This trajectory consists of applying a maximum torque for a period of time to accelerate the robot, followed by an equal period of maximum torque to decelerate it. Mujtaba found that the bang-bang trajectory is executed in minimum time. The quintic, cosine and the sine added to a linear ramp are about $10-20 \%$ slower than the bang-bang trajectory. He also compared a "critically damped" trajectory, composed of a summation of decaying exponentials, in order to approximate the behavior of a damped second order oscillator. He found that this trajectory was three times slower than the others. 
Several groups have approached the optimal joint motion problem as an optimal control problem. Research by Kahn and Roth [20] was involved with determining the forces and torques to input to the robot in order to move from an initial position to a final position in a minimum amount of time. The problem was originally formulated as an optimal control problem. However, for the example worked in this paper, the formulation resulted in twelve highly nonlinear, first order differential equations. In order to solve this problem, it was reduced to a sub-optimal control problem by approximating the nonlinear system with a linearized system and uncoupling the resulting equations. The result is a near-minimum time control of the robot system. Cvetkovic and Vukobratovic [21] also worked on the problem with an optimal control approach. They also determined that the resulting equations are difficult to solve and made approximations to solve the sub-optimal problem. In Vukobratovic's other works mentioned above, $[16],[17]$, he has found that the optimal control theory cannot easily be applied because the system is too complex and highly nonlinear. He has found that the only acceptable approach to the problem is through dynamic programming.

Work has been performed by Nakamichi and Washizu [22], [23], [24], in determining optimal trajectories by formulating the problem as an optimal control problem. However, the problem of interest to them is not that of determining optimal robot joint motions, but problems such as determining an optimal trajectory for a low-thrust rocket in passing between two orbits in space. The optimal trajectory is with respect to minimization of transfer time between the orbits. Their solution approach consists of applying a finite element formulation to solve the optimal control problem. This is done by dividing the time interval 
over which the orbit transfer is to occur into a number of small elements. Shape functions are then assumed for the resulting elements, and the result is a problem of a form which can be solved by a numerical technique which results in the minimum transfer time. Although this approach is not used to solve an optimal robot joint motion problem, the approach used is very similar to that for the robot problem. The method should prove to be successful in its application in the robotics field.

In reviewing the work which has been undertaken in the area of determining joint motions for robot manipulators, it can be seen that a solid foundation exists for determining these motions along end effector paths which are predefined. Approaches are also developed to determine the optimal joint motions along the predefined paths. However, the work that has been done in determining the optimal joint motions along unspecified paths is not developed as completely. The application of optimal control theory seems to prove difficult in solving the resulting equations. However, dynamic programming has been applied with some success. It is the objective of this thesis to contribute to the theory of determining optimal motion programs of robot manipulators in order to move between two points.

\subsection{Proposed Research}

The objective in this thesis is to develop an approach to determine the optimal motion programs at the joints of a robot in order for it to move between two work stations within the robot's workspace. The approach to be developed will be general in nature such that it can be applied to any robot configuration consisting of any number of kinematic links, and of any combination of revolute and prismatic actuators. Also, 
the procedure developed will be able to incorporate various objective functions for determining the optimal motions with only minor modifications to the technique.

The approach developed will be implemented as a computer program. The program will be used to solve several example problems on two link and three link robots with revolute pair actuators. A comparison of the proposed optimal motions for the robot configurations will be made to some of the commonly used motion programs in industry. This will allow the procedure developed in this thesis to be compared and evaluated in order to determine its merit. It is hopeful that this research will provide some insight into the problem of determine optimal joint motions for robots, and provide a background upon which future extensions can be made in this area.

\subsection{Organization}

Chapter 1 of the thesis has provided background information in the area of determining motion programs for robot manipulators. Also, a summary of the objective of the thesis has been given.

Chapter II deals with the development of the theory of solving the optimal joint motion problem. The technique developed is based on formulating the problem as a calculus of variations type problem and solving it with the Rayleigh-Ritz Method. Consideration is also given to the possible objective functions which can be used, and the choice of possible approximating functions to be used in the Rayleigh-Ritz Method.

Chapter 111 applies the theory to a two axis, two dimensional robot. Several factors concerning the solution approach are considered. Comparisons are made in order to determine the desired form of the 
approximating functions for the Rayleigh-Ritz Method. Also, several forms of objective functions are tried and compared. A second problem is solved for the two axis robot which involves changing the position of the task with respect to the robot. Consideration is given to how this effects the optimal motion program. The optimal programs for the two axis robot are also compared to those of a straight line end effector path commonly used in industry.

Chapter IV applies the theory developed in Chapter II to a three link, three revolute robot. The configuration of the robot is such that it moves in three dimensions. The results of the investigations in Chapter 111 are applied in determining the optimal motion program. The optimal motion program obtained is compared to the standard straight line motion.

Chapter V summarizes the work performed in Chapters III and IV and makes some concluding remarks concerning the theory and its application. Comments are made on further work which should be done with the theory developed within this thesis. Also, some ideas are given for extensions of the work in order to develop improved procedures for determining optimal joint motion programs. 
CHAPTER II

\section{THEORY DEVELOPMENT}

\subsection{Definition of the Problem}

The problem confronted in this thesis is that of determining an optimum path for any robot manipulator in moving from a station $A$ to a station B (see Figure 1). For a given manipulator configuration, the motion at each of the robot joints, and in some cases the traveling time from $A$ to to $B$, will be determined which provides an optimum path for the end effector. The motions will be such that they satisfy certain prescribed conditions at the beginning and end of the motion. The optimum path can be calculated with respect to various objective functions. These include minimization of joint torques, energy consumption, etc.

The problem formulation and method of solution on the following pages can be applied to any robot manipulator configuration. However, to provide ease of presentation and understanding, the formulation will be presented for robots having $\mathrm{n}$ links and $\mathrm{n}$ revolute pair actuators. Also, the techniques can be applied to a variety of objective functions, but minimization of joint torques will be: considered in the formulation for the above mentioned reasons. The objective function, I, which minimizes the torque at the robot joints may take the following form:

$$
1=\int_{0}^{T}\left(T_{1}^{2}+T_{2}^{2}+\ldots+T_{n}^{2}\right) d t
$$

where 


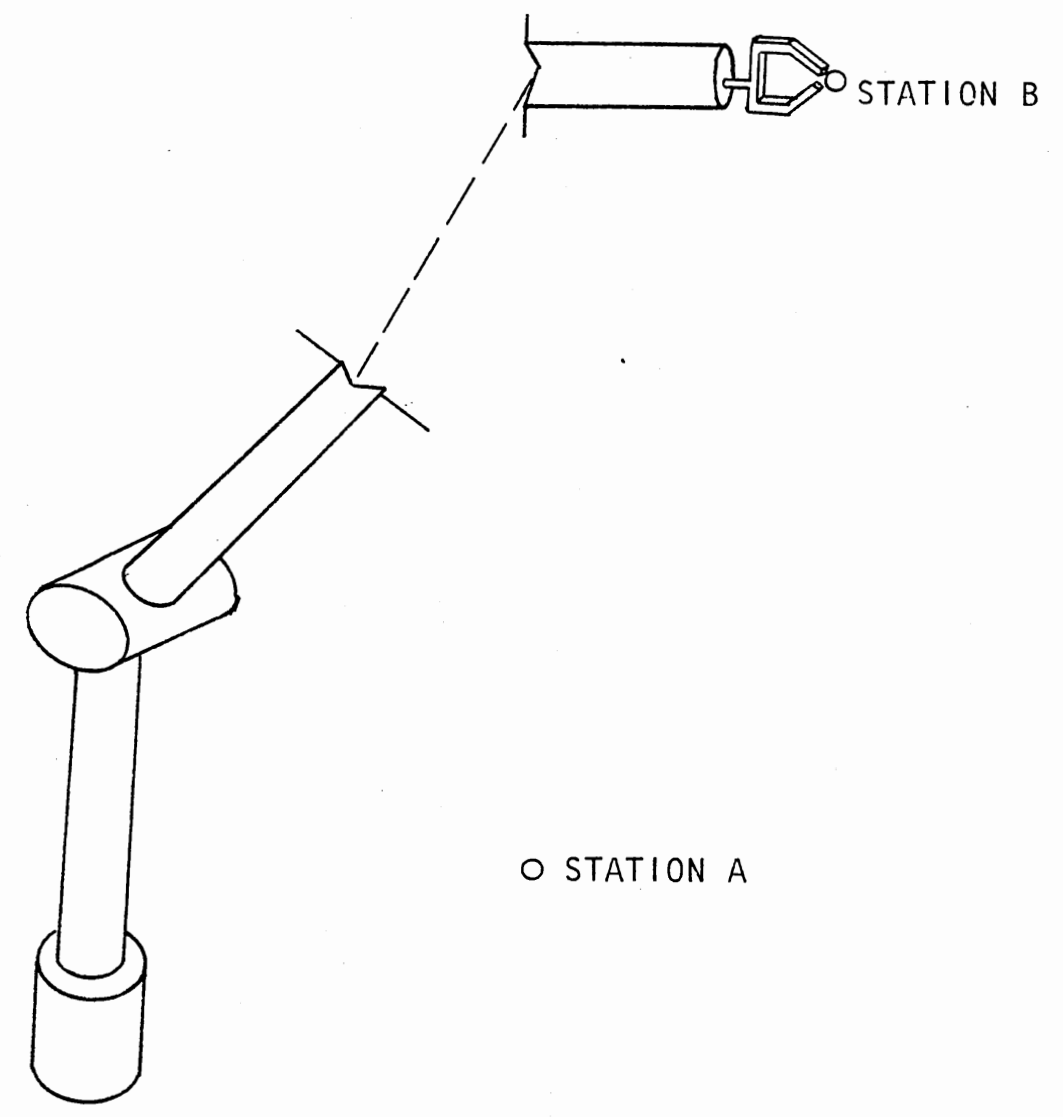

Figure 1. General Robot Configuration 


$$
\begin{aligned}
1 & =\text { objective function; } \\
T_{i} & =\text { torque at joint } i \text {; and } \\
T & =\text { traveling time from } A \text { to } B .
\end{aligned}
$$

\subsection{Formulation of the Problem}

The problem of determining an optimum set of joint motions and, in some cases, an optimum traveling time, can be posed as a calculus of variations problem. The optimal motion program is a set of joint variables which are functions of time that cause the robot to move in an optimal fashion with respect to certain prescribed conditions. The objective in this class of problems is to determine the functions such that a definite integral involving the functions and some of their derivatives will achieve a maximum or minimum value. In the simplest case, the calculus of variations problem takes the form

$$
\underset{\text { or }}{\operatorname{minimize}} \quad I=\int_{x_{1}}^{x_{2}} F\left(x, y, y^{\prime}\right) d x
$$

where $y=y(x)$.

Certain conditions are imposed in determining the maximum or minimum value of the integral. First, certain necessary conditions must be satisfied in order to obtain a maximum or minimum value of 1 . The necessary conditions normally involve setting certain partial derivatives of 1 equal to zero in order to locate the stationary points. Second, certain sufficient conditions are imposed which guarantee that the solution found is an actual maximum or minimum. The sufficient conditions are often not necessary in physically based work, because the solutions can be physically evaluated and interpreted as a maximum or minimum. A further restriction on the problem is that the functions and their derivatives which are 
explicitly involved in the integral I must be continuous over the region of integration.

Examination of the objective function (1) based on minimization of the joint torques indicates that the problem can be posed as a calculus of variations problem.

$$
\text { Minimize } \quad I=\int_{0}^{T}\left(T_{1}^{2}+T_{2}^{2}+\ldots .+T_{n}^{2}\right) d t
$$

where

$$
\begin{aligned}
T_{i}= & T_{i}\left(\theta_{1}(t), \ldots, \theta_{n}(t), \dot{\theta}_{1}(t), \ldots, \dot{\theta}_{n}(t), \ddot{\theta}_{1}(t), \ldots, \ddot{\theta}_{n}(t)\right) \\
i & =1, \ldots, n
\end{aligned}
$$

subject to certain constraints on $\theta_{i}(t), \dot{\theta}_{i}(t)$ at $t=0, T$. For this problem, the unknown functions to be determined are the robot joint displacements, $\theta_{i}(t)$, velocities, $\dot{\theta}_{i}(t)$, and accelerations, $\ddot{\theta}_{i}(t)$, which are all functions of time. The definite integral involved is the sum of the square of the joint torques integrated over the traveling time 0 to $T$.

The problem can also be formulated for other objective functions. These include

$$
\begin{array}{ll}
\operatorname{minimize} & 1=\int_{0}^{T}\left(\left|T_{1}\right|+\left|T_{2}\right|+\ldots+\left|T_{n}\right|\right) d t \\
\text { minimize } \quad 1=\operatorname{maximum}\left\{\left|T_{1}\right|,\left|T_{2}\right|, \ldots,\left|T_{n}\right|\right\} \\
\text { minimize } \quad 1=\int_{0}^{T}\left(T_{1} \dot{\theta}_{1}+T_{2} \dot{\theta}_{2}+\ldots+T_{n} \dot{\theta}_{n}\right) d t
\end{array}
$$

The objective functions in Equations (4) and (5) can also be applied to minimize the joint torques. The difference in the objective functions in Equations (3), (4), and (5) is the manner in which the joint torque and traveling time are involved. Equation (3) involves the square of the joint torques, whereas Equations (4) and (5) involve the joint torques to 
the first power. The traveling time, $T$, is explicitly involved in Equations (3) and (4), but only implicitly involved in Equation (5). The significance of these factors will be considered in the next chapter. The objective function in Equation (6) minimizes the energy consumed by the robot.

\subsection{Method of Solution}

Several methods of solution are available in the calculus of variations problem. The first of these would be the formulation of the Euler equations for the integral $I$. For the simplest case of the calculus of variations problem presented in Equation (2), the Euler equation takes the form

$$
\frac{d}{d x}\left(\frac{\partial F}{\partial y^{1}}\right)-\frac{\partial F}{\partial y}=0
$$

The Euler equation produces a differential equation which can be used to determine the extremals of the problem under consideration. The extremals satisfy the necessary condition of making the integral stationary, but they do not guarantee the existence of a maximum or a minimum.

Formulation of Euler equations does not provide a good solution procedure for the robot motion programming problem because of the number of variable functions involved. Examination of the objective function in Equation (3) indicates that a function $\theta_{i}(t)$ is involved for each of $n$ joints in the robot. Also, the first and second derivatives of each of the joint variables appear in the equation. Thus, not only would an Euler equation have to be developed for each formulation of the objective function, but also the resulting differential equations would be highly nonlinear and difficult to solve. 
The second solution procedure available is that of the Rayleigh-Ritz method [25], [26]. This method provides a procedure for approximating the solution of problems presented in a calculus of variations form. The method performs by assuming that the functions which render the integral I stationary can be approximated by a linear combination of known functions. Thus, assuming that functions $\phi_{i}(t)$ can be properly chosen, the functions to be determined, $\theta_{i}(t)$ can be approximated as

$$
\begin{gathered}
\theta_{i}(t) \stackrel{\simeq}{=} \phi_{0}(t)+c_{i 1} \phi_{1}(t)+c_{i 2} \phi_{2}(t)+\ldots+c_{i m} \phi_{m}(t) \\
i=1, \ldots n
\end{gathered}
$$

The nature of the known functions $\phi_{j}(t)$ is arbitrary, but they must be chosen so as to satisfy certain constraint conditions. $\phi_{0}(t)$ must be chosen to take on the end conditions of $\theta_{i}(t)$ at $t=0$ and $t=T$. For $a$ given tasks for a robot manipulator, the initial and final displacement, $\theta_{i}(0)$ and $\theta_{i}(T)$ of each joint will be known. Also, it is likely that the angular velocity, $\dot{\theta}_{i}(t)$, will be specified at $t=0$ and $t=T$. The function $\phi_{0}(t)$ is chosen accordingly. The remaining functions, $\phi_{1}(t), \phi_{2}(t)$, ..., $\phi_{m}(t)$ are chosen such that they are zero at the end conditions. In this way, only $\phi_{0}(t)$ is operational at the end points, and the boundary conditions of $\theta_{i}(t)$ will be satisfied for any choice of $c_{i j}$ 's.

The nature of the chosen functions $\phi_{j}(t)$ must be such that they form a complete set. That $i s$, some combination of the functions $\phi_{j}(t)$ should be expected to approximate the solution of the problem. Also, the accuracy with which the solution can be approximated should increase with added functions $\phi_{j}(t)$. Again, the nature of the functions chosen will depend on the expected form of the trajectory which solves the physical problem 
at hand. The functions investigated here will be increasing term polynomials and trigonometric terms of increasing harmonics.

Utilizing the Rayleigh-Ritz formulation presented in Equation (8), the calculus of variation problem now takes the form

$$
\text { minimize } \quad 1=\int_{0}^{T}\left(T_{1}^{2}+T_{2}^{2}+\ldots+T_{n}^{2}\right) d t
$$

where

$$
\begin{aligned}
& T_{1}=T_{1}\left(\theta_{1}, \ldots, \theta_{n}, \dot{\theta}_{1}, . ., \dot{\theta}_{n}, \ddot{\theta}_{1}, \ldots, \ddot{\theta}_{n}\right) \\
& \vdots \\
& T_{n}=T_{n}\left(\theta_{1}, \ldots, \theta_{n}, \dot{\theta}_{1}, . ., \dot{\theta}_{n}, \ddot{\theta}_{1}, . ., \ddot{\theta}_{n}\right)
\end{aligned}
$$

and

$$
\begin{aligned}
& \theta_{1}=\theta_{1}\left(t, c_{11}, c_{12}, \ldots, c_{1 m}, T\right) \\
& \vdots \\
& \theta_{n}=\theta_{n}\left(t, c_{n 1}, c_{n 2}, \ldots, c_{n m}, T\right)
\end{aligned}
$$

The problem has now been reduced from finding the functions $\theta_{i}(t)$ which minimize 1 , to determining the time invariant constants $C_{i j}$, and in some cases $T$.

With the equation in the form of Equation (9), the problem can be solved by applying ordinary differential calculus to determine the values of the constants $C_{i j}$ and $T$. The necessary condition for the minimum of the problem is

$$
\frac{\partial I}{\partial C_{i j}}=\int_{0}^{T} 2\left\{T_{1} \frac{\partial T_{1}}{\partial C_{i j}}+T_{2} \frac{\partial T_{2}}{\partial C_{i j}}+\ldots+T_{n} \frac{\partial T_{n}}{\partial C_{i j}}\right\} d t=0
$$

where $i=1, \ldots, n$ and $j=1, \ldots, m$; and

$$
\frac{\partial I}{\partial T}=\frac{\partial}{\partial T} \int_{0}^{T}\left(T_{1}^{2}+T_{2}^{2}+\ldots+T_{n}^{2}\right) d t=0
$$


The result of these partial derivatives is a set of nonlinear algebraic equations which can be solved for the variables $c_{i j}$ and $T$. Once determined, the robot joint trajectories for an optimal path with respect to joint torques will be known.

The choice of the number of terms to use in the Rayleigh-Ritz approximation given by Equation (8) depends upon the nature of the problem. The best approach to use is to solve the problem for the following sequence of approximations and compare the results.

(a) $\theta_{i}(t) \cong \phi_{0}(t)+c_{i l} \phi_{i}(t)$

(b) $\theta_{i}(t) \stackrel{\sim}{=} \phi_{0}(t)+c_{i 1} \phi_{1}(t)+c_{i 2} \phi_{2}(t)$

At some stage the objective function 1 will begin to converge to a minimum value. A point of diminishing return will be established for increasing approximations.

\subsection{Choice of Approximating Functions}

\subsubsection{Polynomials}

In this section, the approximating functions based on polynomials will be developed. As previously mentioned in the preceding section, the functions $\phi_{\mathbf{j}}(\mathrm{t}), \mathrm{j}=0, \ldots, \mathrm{m}$, must satisfy certain boundary conditions. First, the function $\phi_{0}(t)$ will be chosen such that it satisfies the end conditions at each of the robot joints. In moving a robot manipulator from a station $A$ to a station $B$, the configuration of the robot in each of the stations will be known beforehand. Thus, the value of the joint 
angles and values of angular velocity can be specified at each of these stations.

For the robot tasks under consideration in this thesis, it will be required that the manipulator starts from station A with a prescribed velocity and ends at station B with a prescribed velocity. Thus, the end conditions at each joint can be specified as

$$
\begin{array}{ll}
\theta_{i}(0)=\left(\theta_{i}\right)_{0} & \theta_{i}(T)=\left(\theta_{i}\right)_{T} \\
\dot{\theta}_{i}(0)=\left(\dot{\theta}_{i}\right)_{0} & \dot{\theta}_{i}(T)=\left(\dot{\theta}_{i}\right)_{T}
\end{array}
$$

where the traveling time is $T$.

A third order polynomial is necessary to satisfy the four boundary conditions in Equation (13) and takes the form

$$
\begin{aligned}
& \phi_{0}(t)=a_{0}+a_{1} t+a_{2} t^{2}+a_{3} t^{3} \\
& \dot{\phi}_{0}(t)=a_{1}+2 a_{2} t+3 a_{3} t^{2}
\end{aligned}
$$

Thus, the a's must be determined to satisfy the end conditions.

$$
\begin{array}{ll}
\text { @t }=0 & a_{0}=\left(\theta_{i}\right)_{0} \\
a_{1}=\left(\dot{\theta}_{i}\right)_{0} \\
@ t=T \quad a_{0}+a_{1} T+a_{2} T^{2}+a_{3} T^{3}=\left(\theta_{i}\right) T \\
a_{1}+2 a_{2} T+3 a_{3} T^{2}=\left(\dot{\theta}_{i}\right) T
\end{array}
$$

Solving these equations for the values of a yields

$$
\begin{aligned}
& a_{0}=\left(\theta_{i}\right)_{0} \\
& a_{1}=\left(\dot{\theta}_{i}\right)_{0}
\end{aligned}
$$




$$
\begin{aligned}
& a_{2}=\frac{3}{T^{2}}\left\{\left(\theta_{i}\right)_{T}-\left(\theta_{i}\right)_{0}\right\}+\frac{2}{T}\left(\dot{\theta}_{i}\right)_{T} \\
& a_{3}=-\frac{2}{T^{3}}\left\{\left(\theta_{i}\right)_{T}-\left(\theta_{i}\right)_{0}\right\}+\frac{\left(\dot{\theta}_{i}\right)_{0}+\left(\dot{\theta}_{i}\right)_{T}}{T^{2}}
\end{aligned}
$$

Thus,

$$
\begin{aligned}
\phi_{0}(t)= & \left(\theta_{i}\right)_{0}+\left(\theta_{i}\right)_{0} t+\left[\frac{3}{T^{2}}\left[\left(\theta_{i}\right)_{T}-\left(\theta_{i}\right)_{0}\right]+\frac{2}{T}\left(\dot{\theta}_{i}\right)_{T}\right\}^{2} \\
& +\left\{-\frac{2}{T^{3}}\left[\left(\theta_{i}\right)_{T}-\left(\theta_{i}\right)_{0}\right]+\frac{1}{T^{2}}\left[\left(\dot{\theta}_{i}\right)_{0}+\left(\dot{\theta}_{i}\right)_{T}\right]\right\} t^{3}
\end{aligned}
$$

which indeed satisfies the end conditions.

The remaining functions, $\phi_{j}(t), j=1, \ldots, m$, must be chosen such that they vanish at the end conditions and allow $\phi_{0}(t)$ to specify the end conditions. A systematic mathematical procedure to determine these functions is not as easy as for the $\phi_{0}(t)$ function. Therefore, $\phi_{j}(t)$ will be presented and shown to satisfy the following conditions:
(A) $\phi_{j}(\dot{0})=0$
(B) $\phi_{j}(T)=0$
(C) $\dot{\phi}_{j}(0)=0$
(D) $\dot{\phi}_{j}(T)=0$

The polynomial function chosen is of the form

$$
\begin{aligned}
& \phi_{j}(t)=t^{1+j}(T-t)^{2} \\
& \dot{\phi}_{j}(t)=(1+j) t^{j}(T-t)^{2}-2 t^{j+1}(T-t)
\end{aligned}
$$

This function $\phi_{j}(t)$ provides for polynomials of increasing degree as $j$ varies from 1 to $m$. All terms in the function $\phi_{j}(t)$ and $\dot{\phi}_{j}(t)$ contain the variable $t$ which will allow them to satisfy the end conditions ( $A$ ) 
and (C) of Equation (19). Also, all terms of Equations (20) and (21) contain the expression $(T-t)$ allowing end conditions (B) and (D) of Equation (19) to be satisfied at $t=T$.

The resulting approximating function for the joint variables takes the following form: ;

$$
\begin{aligned}
\theta_{i}(t) \stackrel{\sim}{=} & \left(\theta_{i}\right)_{0}+\left(\dot{\theta}_{i}\right)_{0} t+\left[\frac{3}{T^{2}}\left[\left(\theta_{i}\right)_{T}-\left(\theta_{i}\right)_{0}\right]+\frac{2}{T}\left(\dot{\theta}_{i}\right)_{T}\right\} t^{2} \\
& +\left[-\frac{2}{T^{3}}\left[\left(\theta_{i}\right)_{T}-\left(\theta_{i}\right)_{0}\right]+\frac{1}{T^{2}}\left[\left(\dot{\theta}_{i}\right)_{0}+\left(\dot{\theta}_{i}\right)_{T}\right]\right]_{t}{ }^{3} \\
& +\sum_{j=1}^{m} c_{i j} t^{1+j}(T-t)^{2} \quad i=1, \ldots, n
\end{aligned}
$$

The graphs shown in Figure 2 show the first four terms of $\phi_{j}(t)$ which will be used in approximating $\theta_{i}(t)$.

\section{$\underline{2.4 .2}$ Trigonometrics}

The use of trigonometric functions will also be investigated for their potential as approximating functions. The form will be sine and cosine terms of increasing harmonics. The function $\phi_{0}(t)$ which is chosen to satisfy the boundary conditions of $\theta_{i}(t)$ can remain in the form of Equation (18). A derivation similar to that presented for the polynomial functions must be undertaken to provide the $\phi_{j}(t)$ to satisfy the conditions in Equation (19). The resulting function takes the following form:

$$
\begin{aligned}
& \phi_{j}(t)=1-\cos \frac{2 j^{\pi t}}{T} \\
& \dot{\phi}_{j}(t)=\frac{{ }^{2} j^{\pi}}{T} \sin \frac{{ }^{2} j^{\pi t}}{T}
\end{aligned}
$$

The resulting form of $\theta_{i}(t)$ is 


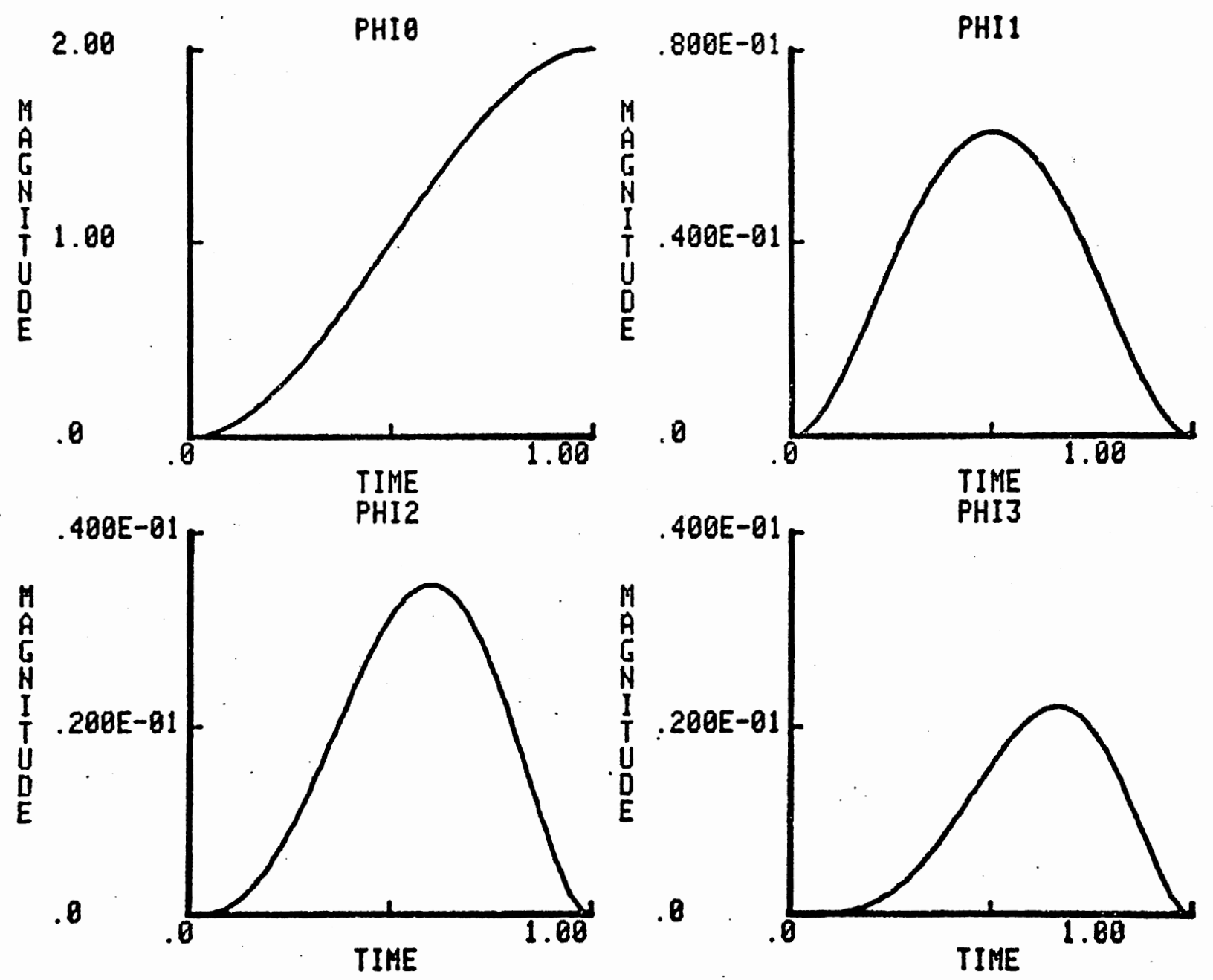

Figure 2. Polynomial Functions of Increasing Order 


$$
\begin{aligned}
\theta_{i}(t)= & \left(\theta_{i}\right)_{0}+\left(\dot{\theta}_{i}\right)_{0} t+\left\{\frac{3}{T^{2}}\left[\left(\theta_{i}\right)_{T}-\left(\theta_{i}\right)_{0}\right]+\frac{2}{T}\left(\dot{\theta}_{i}\right)_{T}\right\} t^{2} \\
& +\left\{-\frac{2}{T^{3}}\left[\left(\theta_{i}\right)_{T}-\left(\theta_{i}\right)_{0}\right]+\frac{1}{T^{2}}\left[\left(\dot{\theta}_{i}\right)_{0}+\left(\dot{\theta}_{i}\right)_{T}\right] t^{3}\right. \\
& +\sum_{j=1}^{m} c_{i j}\left\{1-\cos \frac{2 j \mathrm{j}}{T}\right\} \quad i=1, \ldots, n
\end{aligned}
$$

The graphs in Figure 3 show the first four terms of $\phi_{j}(t)$ of the trigonometric form.

\subsection{Computational Procedure}

An algorithm must be obtained for solving the set of nonlinear algebraic equations resulting from the partial derivatives (10) and (11) in order to obtain the weighting constants $C_{i j}$ and traveling time T. A suit-

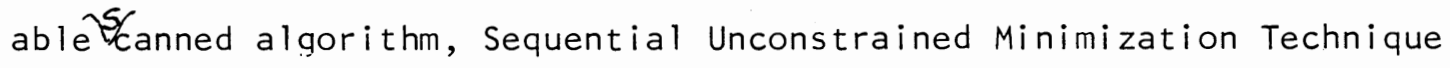
(SUMT), was chosen because it is easily accessible on the OSU computer system. The actual formulation of the problem input to SUMT is not of the form of the nonlinear algebraic equations which result from Equations (10) and (11). However, the procedure used to solve the input problem is equivalent to solving the set of nonlinear algebraic equations.

The SUMT algorithm was developed by Fiacco and McCormick [27]. The mathematical programming problem to be solved by the algorithm is to determine a vector $\bar{x}$ that solves

$$
\text { Minimize } f(\bar{x})
$$

subject to

$$
\begin{aligned}
& g_{j}(\bar{x})>0, \quad j=1,2, \ldots, m \\
& h_{j}(\bar{x})=0, \quad j=m+1, \ldots, m+p
\end{aligned}
$$



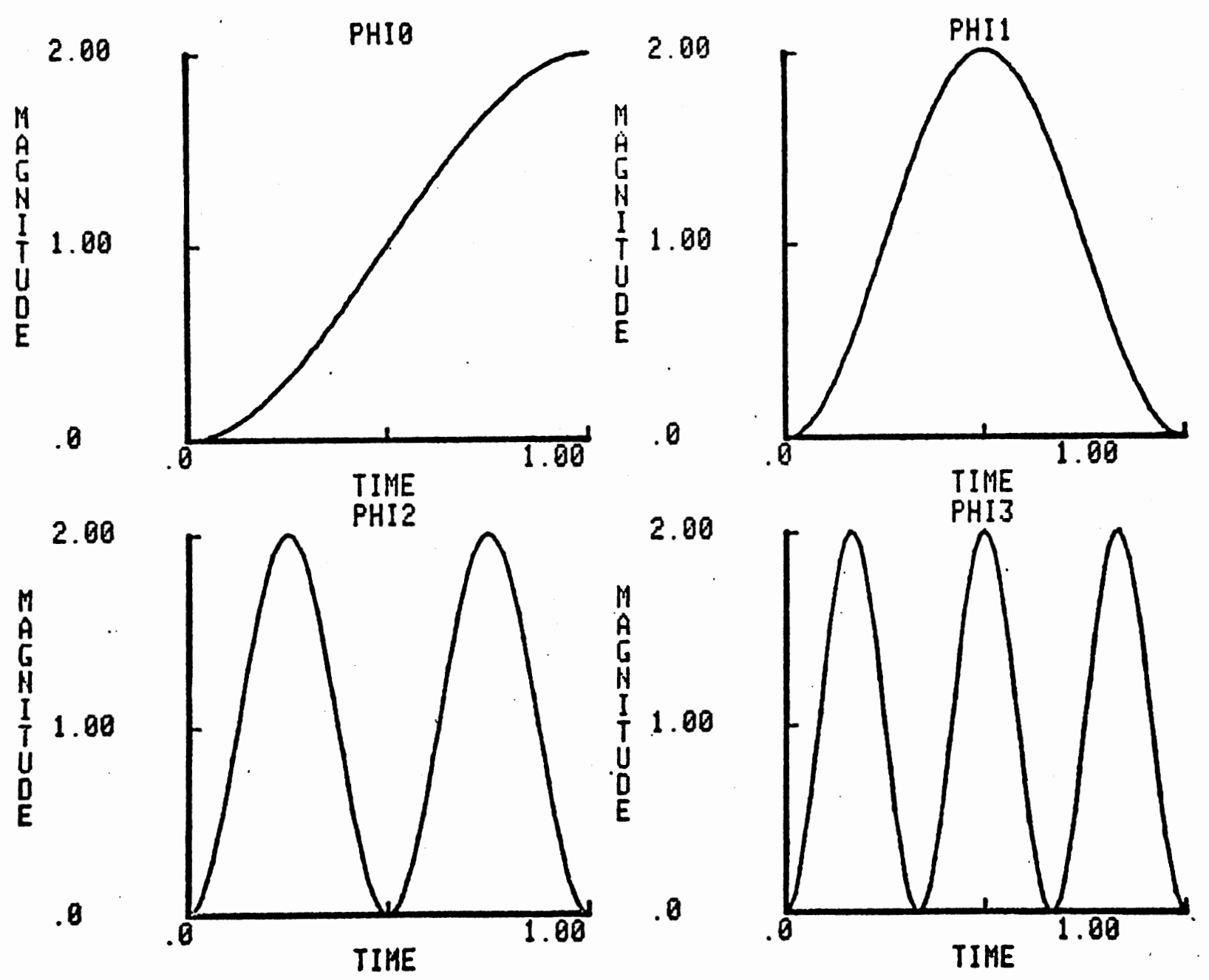

Figure 3. Trigonometric Functions of Increasing Harmonics 
The idea utilized in the algorithm is to solve a sequence of unconstrained problems whose solution approach that of the solution of the constrained problem in Equation (26). An unconstrained problem, $P(x, r)$, is formed by including the constraints as penalty functions within the objective function. The function $P(x, r)$ is defined as

$$
P(x, r)=f(x)-r \sum_{j=1}^{m} \ln g_{j}(x)+\sum_{j=m+1}^{m+P} \frac{\left[h_{j}(x)\right]^{2}}{r}
$$

This procedure requires minimization of $P(x, r)$ over those $x$ satisfying the conditions $g_{j}(x)>0, j=1, \ldots, m$ for $r=r 1, r 2, \ldots$, where $r 1>r 2$ $>\ldots>r k>\ldots>0$. Under suitable conditions the minima of $P$ represented by $x(r 1), x(r 2), \ldots x(r k), \ldots$ exist and approach a solution of Equation (26) as $r \rightarrow 0$.

The SUMT algorithm gives the option of several different procedures for solving the minimization problem. These methods differ in their use of gradient vectors and the Hessian matrix (matrix of second partial derivatives). The method utilized in solving the manipulator trajectory problem was Newton's method which takes the form

$$
\bar{x}^{i+1}=\bar{x}^{i}-\lambda^{i}\left[\nabla^{2} f\left(\bar{x}^{-i}\right)\right]^{-1} \nabla f\left(\bar{x}^{-i}\right)
$$

Newton's method starts at a current point $\bar{x}^{i}$ and searches along the gradient vector $\nabla f\left(\bar{x}^{i}\right)$ as modified by the Hessian matrix $\nabla^{2} f\left(\bar{x}^{i}\right)$ in order to locate an improved minimum point $\bar{x}^{i+1}$.

This procedure is equivalent to solving a set of nonlinear algebraic equations. The solution procedure formulated in Equations (10) and (11) involved setting the partial derivatives of $I$ with respect to $C_{i j}$ and $T$ equal to zero and solving for $C_{i j}$ and $T$. The collection of partial derivatives would represent the gradient of 1 . For the moment, let this 
gradient be denoted by $\nabla f\left(\bar{x}^{i}\right)$. It is desired to find the roots of $\nabla f\left(\bar{x}^{i}\right)$ such that the objective function $I$ is stationary. The roots of $\nabla f\left(\bar{x}^{i}\right)$ can be represented by a vector $\bar{x}$ which contains the constants $c_{i j}$ and $T$. Assuming a point $\bar{x}^{i}$ is known which is close to a root of $\nabla f(\bar{x})$, an improved value of $\bar{x}^{i}, \bar{x}^{i+l}$ can be obtained by examining a Taylor series expansion to two terms.

$$
\nabla f\left(\bar{x}^{i+1}\right) \cong \nabla f\left(\bar{x}^{i}\right)+\left(\bar{x}^{i+1}-\bar{x}^{-i}\right) \nabla^{2} f\left(\bar{x}^{-i}\right)
$$

For $\bar{x}^{i+1}$ to be a root of $\nabla f(\bar{x}), \nabla f\left(\bar{x}^{i+1}\right)=0$. Thus

$$
\nabla f\left(x^{-i}\right)+\left(\bar{x}^{i+1}-\bar{x}^{-i}\right) \nabla^{2} f\left(\bar{x}^{i}\right)=0
$$

and

$$
\bar{x}^{i+1}=\bar{x}^{i}-\left[\nabla^{2} f\left(\bar{x}^{i}\right)\right]^{-1} \nabla f\left(\bar{x}^{-i}\right)
$$

Comparing Equations (28) and (31) shows that the expressions are equivalent for $\lambda^{i}=1$. Thus, the application of Newton's method in minimizing an unconstrained objective function is equivalent to solving a set of nonlinear algebraic equations. Thus, it is acceptable to solve the manipulator joint motion problem using SUMT.

The logic of the SUMT algorithm is as follows:

Step 1: Find a point $x^{\circ}$ in $R^{0}=\left\{x / g_{i}(x)>0, i=1, \ldots m\right\}$. If such a point is not readily available, the optimization method itself is used to find it.

Step 2: Determine $r_{1}$, the initial value of $r$. This can be specified as an input parameter or one of several rules for choosing $r_{1}$ can be specified.

Step 3: Determine the minimum of the $P$ function for the current value of $r$ using one of the several procedures included in the computer program. 
Step 4: Estimate the solution using extrapolation formulas.

Step 5: Terminate computations if final convergence criteria are satisfied using bounds on the optimal solution obtained from the theory of duality.

Step 6: Select $\mathrm{r}_{\mathrm{k}+1}$.

Step 7: Go to Step 3.

A simplified flow diagram of the algorithm is shown in Figure 4. 


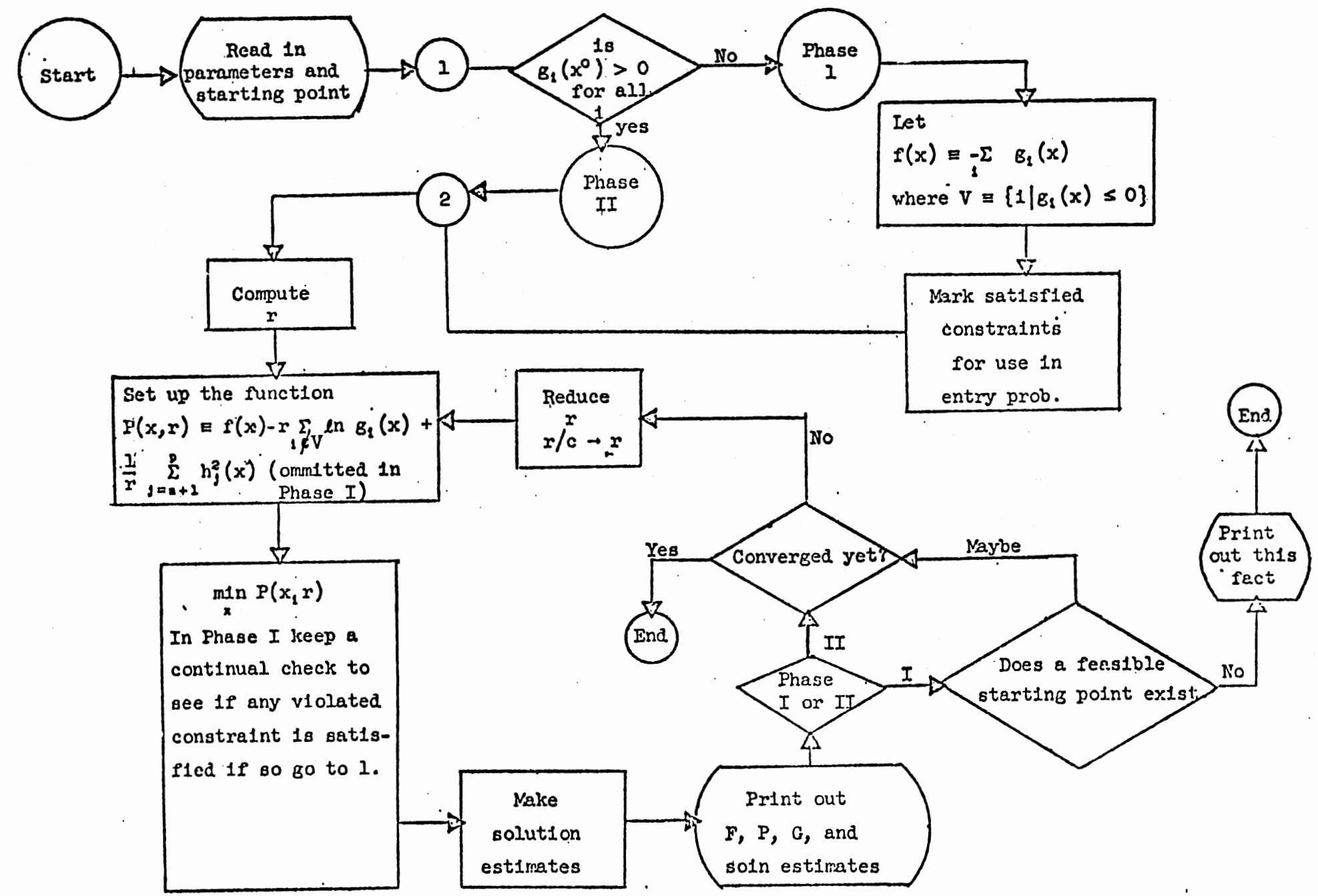

Figure 4. Simplifi.ed Flow Chart of the SIIMT Alaorithm 
CHAPTER 111

APPLICATION OF THE THEORY TO A 2-R ROBOT

\subsection{Scope of Chapter}

In this chapter the SUMT computer algorithm will be applied to optimize the joint motions of a two link, two dimensional robot manipulator. Several factors involved with the solution procedure of this problem will be investigated to determine the best approach for solving optimal joint motion problems. The factors to be considered are listed below:

1. Determination of the number of trigonometric terms required in the approximating functions.

2. Comparison of polynomial and trigonometric approximating functions.

3. Procedure to determine the optimal traveling time, $T$.

4. Comparison of objective functions.

5. Comparison of optimal and standard solutions.

6. Comparison of workstation locations with respect to the manipulator.

\subsection{Problem to be Solved}

The task to be performed by the manipulator is a simple lifting task from station A to station B (see Figure 5). The manipulator joints will be required to start at station $A$ with zero angular velocity, and end at 


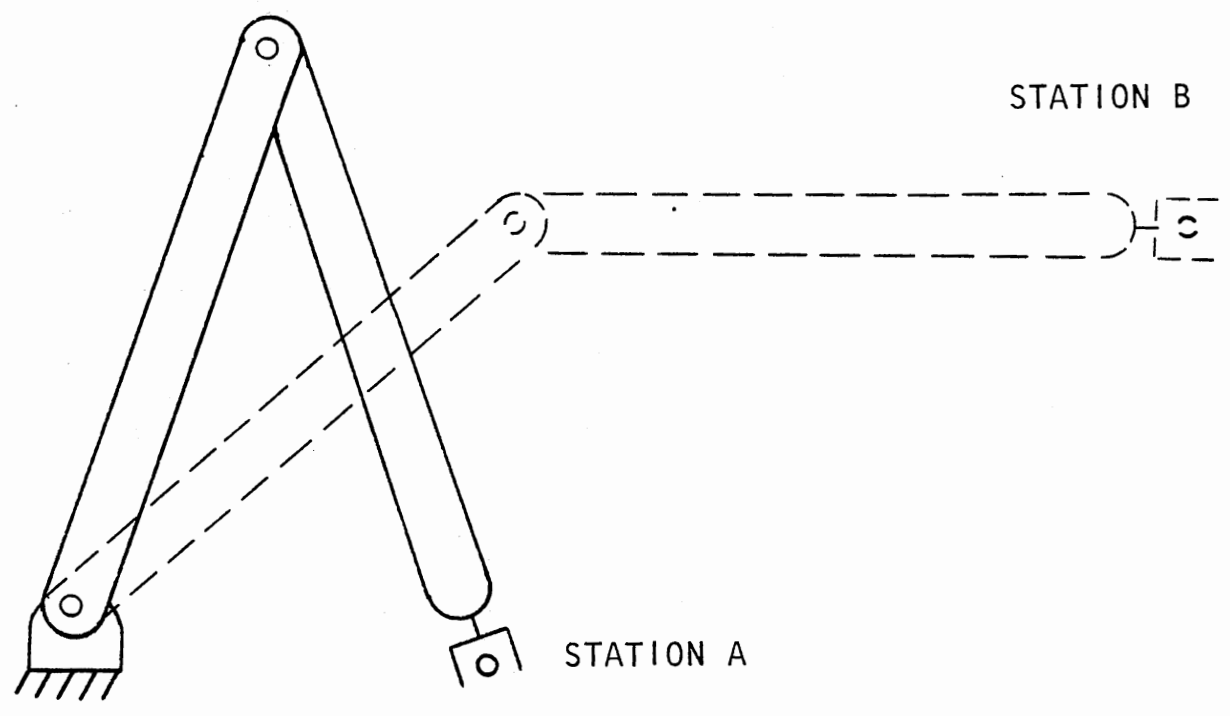

Figure 5. Two Axis Robot Task Description 
station B with zero angular velocity. Thus, the boundary conditions are specified.

$$
\begin{array}{ll}
\theta_{1}(0)=70.0^{\circ} & \theta_{2}(0)=210.0^{\circ} \\
\theta_{1}(t)=45.0^{\circ} & \theta_{2}(T)=315.0^{\circ} \\
\dot{\theta}_{1}(0)=0.0 & \dot{\theta}_{2}(0)=0.0 \\
\dot{\theta}_{1}(t)=0.0 & \dot{\theta}_{2}(t)=0.0
\end{array}
$$

Figure 6 shows a free-body diagram of the two axis robot in which the variables are described. The variable values chosen for the example problem in this chapter are listed below:

$$
\begin{array}{ll}
a_{1}=0.6095 \mathrm{~m} & a_{2}=0.6096 \mathrm{~m} \\
l_{1}=0.3048 \mathrm{~m} & l_{2}=0.3048 \mathrm{~m} \\
M_{1}=4.5360 \mathrm{~kg} & M_{2}=6.3500 \mathrm{~kg} \\
I_{1}=0.1405 \mathrm{~kg} \cdot \mathrm{m}^{2} & l_{2}=0.2458 \mathrm{~kg} \cdot \mathrm{m}^{2}
\end{array}
$$

Note: The inertial properties of the load carried at the end effector are included in the second link.

The objective function to be evaluated is that of minimizing the integral of the sum of the square of the joint torques.

$$
\operatorname{minimize} \quad I=\int_{0}^{T}\left(T_{1}^{2}+T_{2}^{2}\right) d t
$$

The kinematic parameters of the two axis robot must be determined in order that the objective function can be evaluated. Expressions for the position, velocity and acceleration of the point $P$ on the end of the second link are required. These equations are 1 isted below and are measured and expressed in terms of an inertial reference frame at $0_{1}$. 


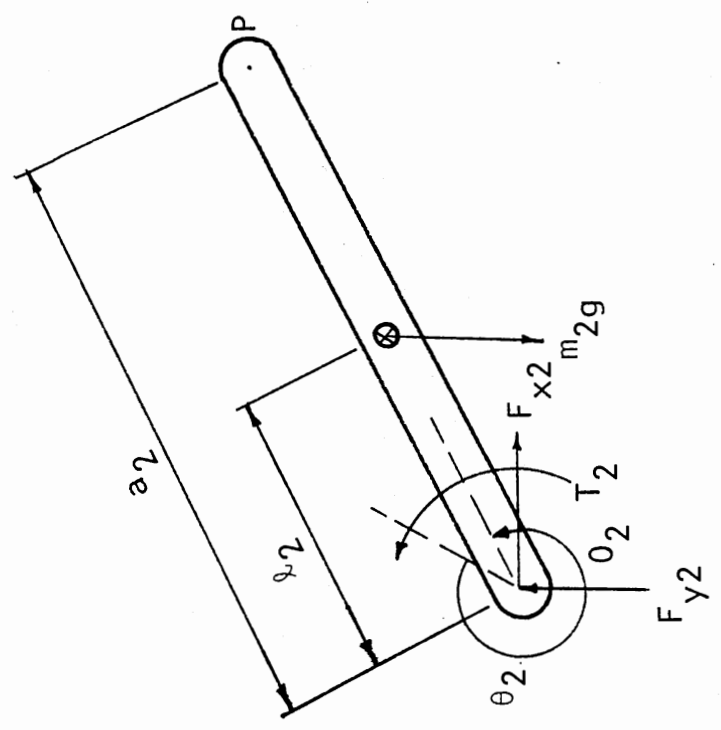

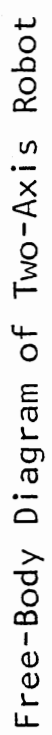

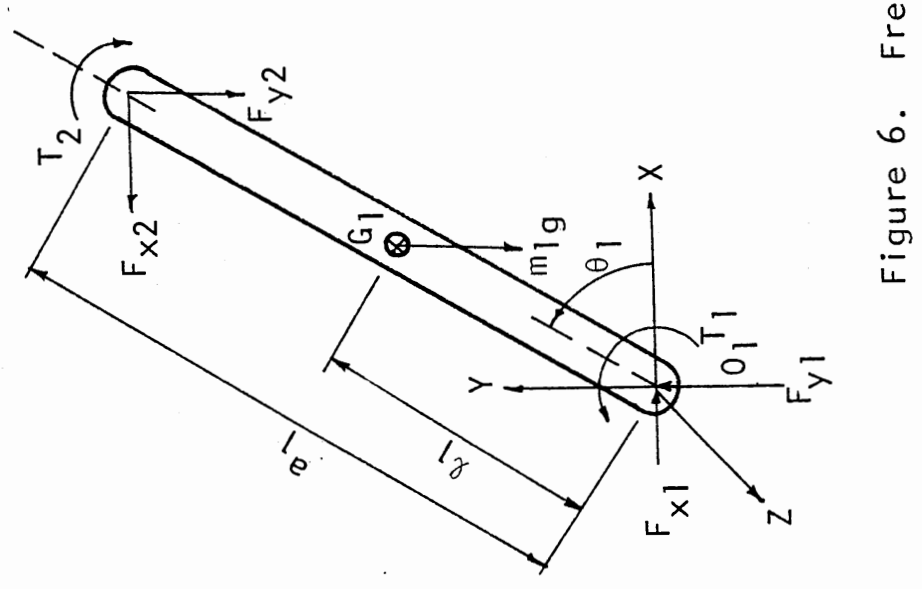




$$
\begin{aligned}
& x_{p}=a_{1} \cos \theta_{1}+a_{2} \cos \left(\theta_{1}+\theta_{2}\right) \\
& y_{p}=a_{1} \sin \theta_{1}+a_{2} \sin \left(\theta_{1}+\theta_{2}\right)
\end{aligned}
$$

$\dot{x}_{p}=-\left[a_{1} \sin \theta_{1}+a_{2} \sin \left(\theta_{1}+\theta_{2}\right)\right] \dot{\theta}_{1}-\left[a_{2} \sin \left(\theta_{1}+\theta_{2}\right)\right] \dot{\theta}_{2}$

$\dot{y}_{p}=\left[a_{1} \cos \theta_{1}+a_{2} \cos \left(\theta_{1}+\theta_{2}\right)\right] \dot{\theta}_{1}+\left[a_{2} \cos \left(\theta_{1}+\theta_{2}\right)\right] \dot{\theta}_{2}$

$\ddot{x}_{p}=-\left[a_{1} \cos \theta_{1}+a_{2} \cos \left(\theta_{1}+\theta_{2}\right)\right] \dot{\theta}_{1}^{2}-\left[2 a_{2} \cos \left(\theta_{1}+\theta_{2}\right)\right] \dot{\theta}_{1} \dot{\theta}_{2}$

$-\left[a_{2} \cos \left(\theta_{1}+\theta_{2}\right)\right] \dot{\theta}_{2}^{2}-\left[a_{1} \sin \theta_{1}+a_{2} \sin \left(\theta_{1}+\theta_{2}\right)\right] \ddot{\theta}_{1}$

$-\left[a_{2} \sin \left(\theta_{1}+\theta_{2}\right)\right] \ddot{\theta}_{2}$

$$
\begin{aligned}
\ddot{y}_{p}= & -\left[a_{1} \sin \theta_{1}+a_{2} \sin \left(\theta_{1}+\theta_{2}\right)\right] \dot{\theta}_{1}^{2}-\left[2 a_{2} \sin \left(\theta_{1}+\theta_{2}\right)\right] \dot{\theta}_{1} \dot{\theta}_{2} \\
& -\left[a_{2} \sin \left(\theta_{1}+\theta_{2}\right)\right] \dot{\theta}_{2}^{2}+\left[a_{1} \cos \theta_{1}+a_{2} \cos \left(\theta_{1}+\theta_{2}\right)\right] \ddot{\theta}_{1} \\
& +\left[a_{2} \cos \left(\theta_{1}+\theta_{2}\right)\right] \ddot{\theta}_{2}
\end{aligned}
$$

Expressions for the accelerations, $a_{G l}$ and $a_{G 2}$, of the centers of gravity of 1 inks 1 and 2 , and the reaction forces at the joints are also required. These are necessary to evaluate the joint torque expressions, and are included in the joint torque expressions presented below.

Expressions for the torques at each joint of the robot are required in order to evaluate the objective function in Equation (1). The joint torque expressions take the following form:

$$
\begin{aligned}
T_{2}= & 1_{2}\left(\ddot{\theta}_{1}+\ddot{\theta}_{2}\right)+m_{2}\left[a_{1}\left(\ddot{\theta}_{1} \sin \theta_{1}+\dot{\theta}_{1}^{2} \cos \theta_{1}\right)\right. \\
& +l_{2}\left\{\left(\ddot{\theta}_{1}+\ddot{\theta}_{2}\right) \sin \left(\theta_{1}+\theta_{2}\right)\right. \\
& \left.\left.+\left(\dot{\theta}_{1}+\dot{\theta}_{2}\right)^{2} \cos \left(\theta_{1}+\theta_{2}\right)\right\}\right] l_{2} \sin \left(\theta_{1}+\theta_{2}\right) \\
& +m_{2}\left[g+a_{1}\left(\ddot{\theta}_{1} \cos \theta_{1}-\dot{\theta}_{1}^{2} \sin \theta_{1}\right)\right.
\end{aligned}
$$




$$
\begin{aligned}
&+ l_{2}\left\{\left(\ddot{\theta}_{1}+\ddot{\theta}_{2}\right) \cos \left(\theta_{1}+\theta_{2}\right)\right. \\
&\left.\left.-\left(\dot{\theta}_{1}+\dot{\theta}_{2}\right)^{2} \sin \left(\theta_{1}+\theta_{2}\right)\right\}\right] l_{2} \cos \left(\theta_{1}+\theta_{2}\right) \\
& T_{1}= T_{2}+1_{1} \ddot{\theta}_{1}+\left(a_{1} \sin \theta_{1}\right) m_{2}\left[a_{1} \ddot{\theta}_{1} \sin \theta_{1}\right. \\
&+ l_{2}\left(\ddot{\theta}_{1}+\ddot{\theta}_{2}\right) \sin \left(\theta_{1}+\theta_{2}\right)+a_{1} \dot{\theta}_{1}^{2} \cos \theta_{1} \\
&+\left.l_{2}\left(\dot{\theta}_{1}+\dot{\theta}_{2}\right)^{2} \cos \left(\theta_{1}+\theta_{2}\right)\right] \\
&+\left(a_{1} \cos \theta_{1}\right) m_{2}\left[g+a_{1} \ddot{\theta}_{1} \cos \theta_{1}\right. \\
&+l_{2}\left(\ddot{\theta}_{1}+\ddot{\theta}_{2}\right) \cos \left(\theta_{1}+\theta_{2}\right)-a_{1} \dot{\theta}_{1}^{2} \sin \theta_{1} \\
&\left.-l_{2}\left(\dot{\theta}_{1}+\dot{\theta}_{2}\right)^{2} \sin \left(\theta_{1}+\theta_{2}\right)\right] \\
&+m_{1} l_{1}^{2} \sin \theta_{1}\left(\dot{\theta}_{1}^{2} \cos \theta_{1}+\ddot{\theta}_{1} \sin \theta_{1}\right) \\
&+m_{1} l_{1}^{2} \cos \theta_{1}\left(\frac{g}{l_{1}}-\dot{\theta}_{1}^{2} \sin \theta_{1}+\ddot{\theta}_{1} \cos \theta_{1}\right)
\end{aligned}
$$

Thus, once the joint angular displacements, velocities and accelerations are specified for a given instant of time, the objective function I can be evaluated.

Before presenting the results of these investigations, a comment must be made concerning the approach which must be undertaken in solving problems of this type with SUMT. The problem presented in Equation (1) is referred to as a non-convex programming problem. This means that there is no guarantee that SUMT will converge to the global minimum of the problem, only that local minima will be achieved. Thus, several starting points must be used for the optimization process. In taking a well distributed set of starting points, it is hoped that a trend can be observed in the resulting minima so that an optimal solution can be stated with some confidence. 


\subsection{Determination of Required Number of Terms}

The first factor considered was that of determining the number of terms required in approximating the optimal joint motion. Trigonometric approximating functions were used. The approach used here was to solve the problem for a sequence of approximations with increasing terms and determine when increasing terms provide no improvement. The approximations are of the form:

$$
\theta_{i}(t) \cong \phi_{0}(t)+C_{i l} \phi_{1}(t)
$$

(2) $\theta_{i}(t) \cong \phi_{0}(t)+c_{i 1} \phi_{1}(t)+c_{i 2} \phi_{2}(t)$

(3) $\theta_{i}(t) \cong \phi_{0}(t)+c_{i 1} \phi_{1}(t)+c_{i 2 \dot{\phi}_{2}}(t)+c_{i 3} \phi_{3}(t)$

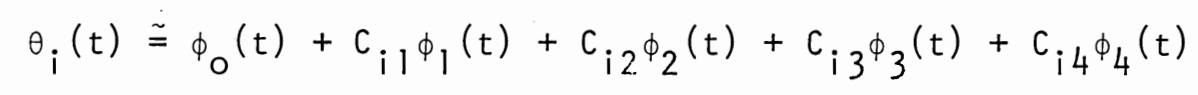

Traveling time, $T$, was not optimized but constrained to a fixed value. The SUMT algorithm will be used only to determine the $c_{i j}$ of the approximating functions. Although the problem of local minima has been recognized and presents a need for multiple starting points, only one starting point will be used here. This will be such that all $c_{i j}=0$. This starting point specifies the $\phi_{0}(t)$ function as the initial motion at each joint. It is known beforehand that this function satisfies the initial conditions and provides a smooth motion at each joint (see figure 3 ). Also, the $C_{i j}=0$ starting point provides a direct path for the end effector in traveling from $A$ to $B$. All of these facts have been verified in a preliminary investigation and will not be preaented in this thesis.

The sequence of approximations in Equation (10) was performed for $T=0.45$ seconds. Table I summarizes the results. In Table I, the weighting coefficients of the approximating functions and the objective function 
TABLE I

COMPARISON OF INCREASING TERM TRIGONOMETRIC APPROXIMATIONS AT $T=0.45$ SECONDS

\begin{tabular}{|c|c|c|c|c|c|}
\hline \multirow{2}{*}{$\begin{array}{l}\begin{array}{l}\text { No. of } \\
\text { Terms }\end{array} \\
0\end{array}$} & \multicolumn{2}{|c|}{ Starting Point } & \multicolumn{2}{|c|}{ Converged Point } & \multirow{2}{*}{$\frac{\text { Objective Value }}{1583.0}$} \\
\hline & \multicolumn{2}{|c|}{---} & $c_{11}=0.0$ & $c_{21}=0.0$ & \\
\hline 1 & $c_{11}=0.0$ & $c_{21}=0.0$ & $c_{11}=0.073700$ & $c_{21}=-0.116500$ & 1220.0 \\
\hline \multirow{2}{*}{2} & $c_{11}=0.0$ & $c_{21}=0.0$ & $c_{11}=0.074200$ & $c_{21}=-0.115600$ & \multirow{2}{*}{1218.8} \\
\hline & $c_{12}=0.0$ & $c_{22}=0.0$ & $c_{12}=-0.000593$ & $c_{22}=-0.000223$ & \\
\hline \multirow{3}{*}{3} & $c_{11}=0.0$ & $c_{21}=0.0$ & $c_{11}=0.0743$ & $c_{21}=-0.115600$ & \multirow{3}{*}{1218.5} \\
\hline & $c_{12}=0.0$ & $c_{22}=0.0$ & $c_{12}=-0.000630$ & $C_{22}=-0.000287$ & \\
\hline & $c_{13}=0.0$ & $c_{23}=0.0$ & $c_{13}=0.000257$ & $c_{23}=-0.000361$ & \\
\hline \multirow{4}{*}{4} & $c_{11}=0.0$ & $c_{21}=0.0$ & $c_{11}=-0.074300$ & $c_{21}=-0.116200$ & \multirow{4}{*}{1218.0} \\
\hline & $c_{12}=0.0$ & $c_{22}=0.0$ & $c_{12}=-0.000632$ & $c_{22}=-0.000159$ & \\
\hline & $c_{13}=0.0$ & $c_{23}=0.0$ & $c_{13}=0.000161$ & $c_{23}=-0.000073$ & \\
\hline & $c_{14}=0.0$ & $c_{24}=0.0$ & $c_{14}=0.000028$ & $c_{24}=-0.000117$ & \\
\hline
\end{tabular}


value are given for the increasing term solutions. The column labeled "number of terms" can be interpreted as the number of terms which have been added to the $\phi_{0}(t)$ solution. In examining the table, it can be seen that a considerable improvement is obtained in going from the $\phi_{0}(t)$ function to a solution with one term added to it. However, the solutions for approximating functions with more than one term added do not show any significant improvement over that for the one term solution. This fact is shown graphically in Figures 7 and 8 . Figure 7 compares the motion at joint one of the robot for the zero term through the three term solutions (OT = zero terms added to $\phi_{0}(t)$, etc.). It can easily be seen here that the one term solution is significantly different from that of the zero term solution, but increasing term solutions give the same results. Figure 8 shows a similar comparison for the second joint of the robot. Figures 9 and 10 compare the torques at joints one and two for the zero term and one term solutions. Figure 9 shows that the torque profile at joint one is much smoother and has lower peaks for the one term solution than the zero term solution. This was expected for the lower value of objective function achieved. Figure 10 compares the two solutions for the second joint. The improvement here is not as significant, but it can be seen that the one term solution has lower values of peak torque. Figure 11 shows a comparison of the two solutions with respect to the resulting end effector path. It can be seen here that the one term solution provides the added advantage of providing a more direct path from station $A$ to station B than that for the zero term solution.

The optimal joint motion at $T=0.45$ seconds can be accurately approximated by adding one trigonometric term to the $\phi_{0}(t)$ solution. In order to determine if this case is true in general, the same approach was 


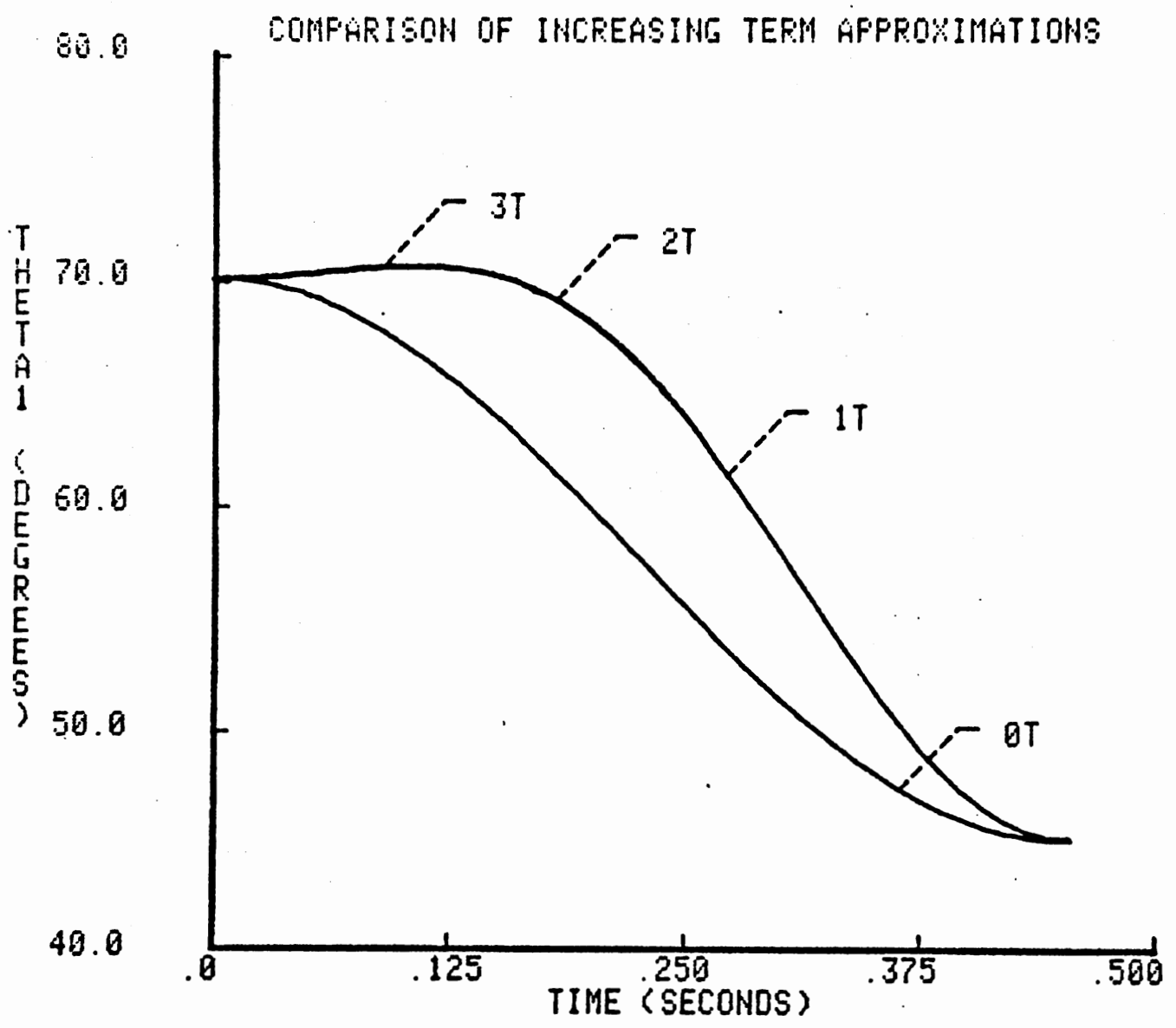

Figure 7. Comparison of Increasing Term Trigonometric Approximations of Displacement of Joint 1 . 


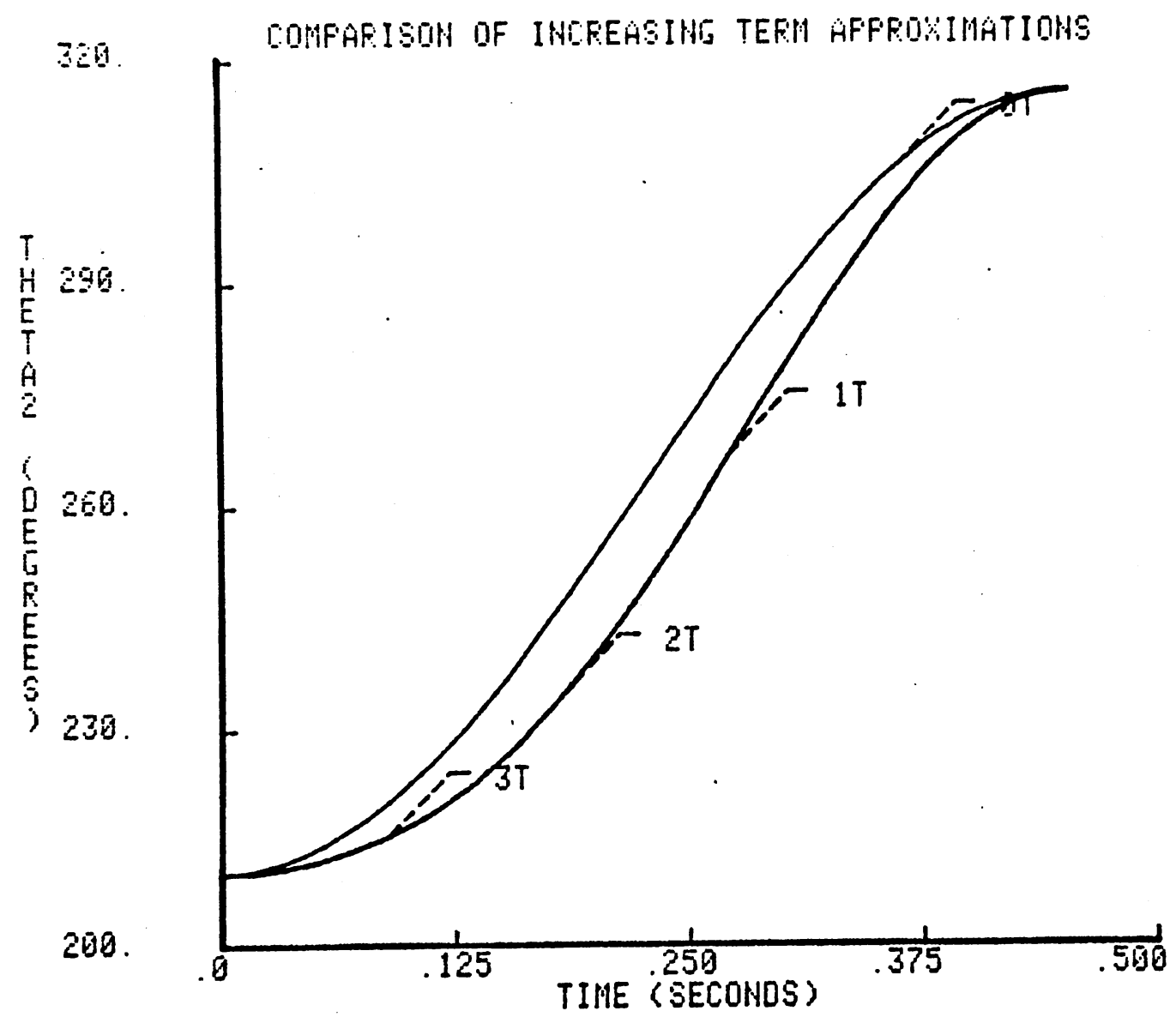

Figure 3. Comparison of Increasing Term Trigonometric Approximations of Displacement of Joint 2 . 


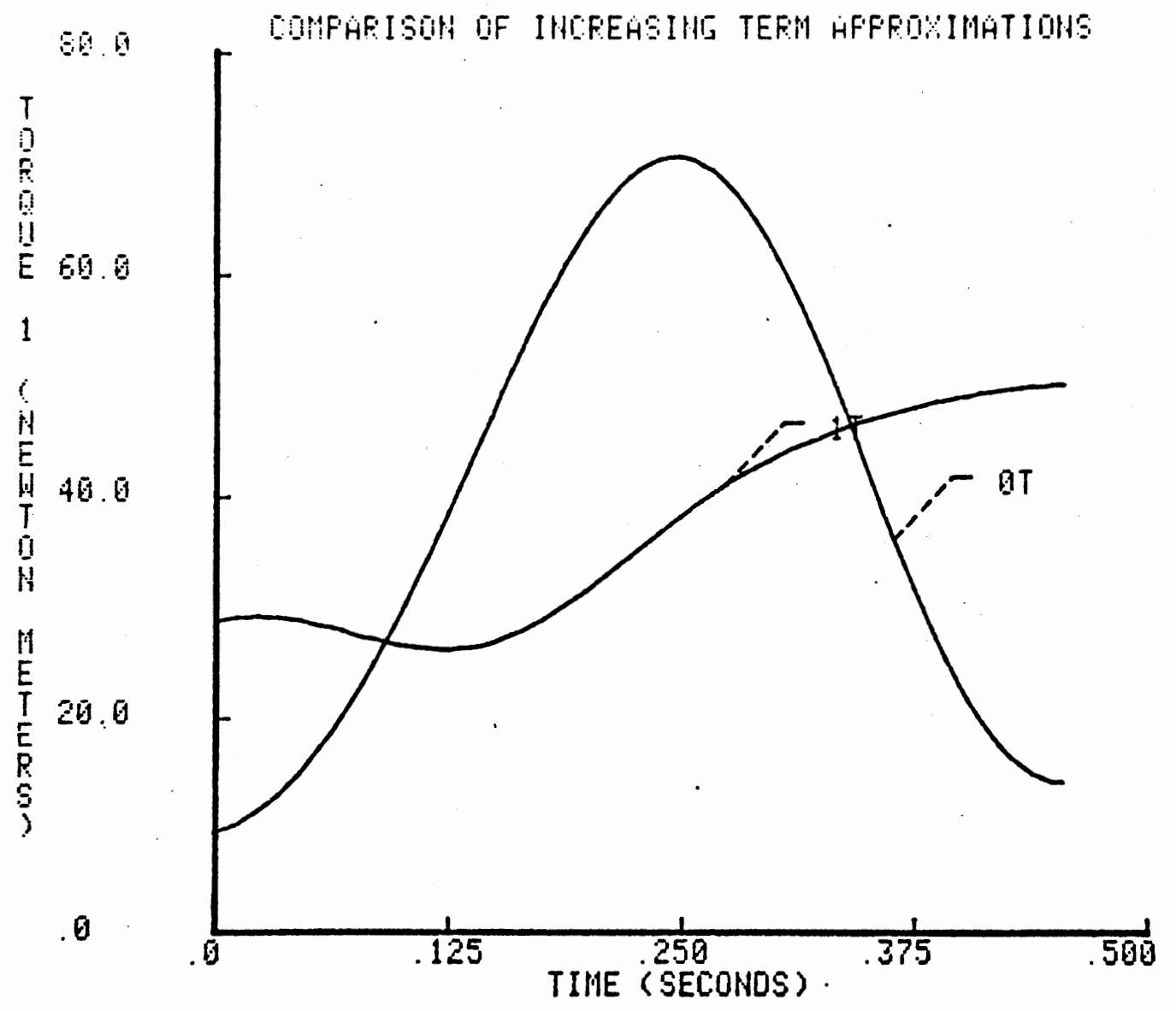

Figure 9. Comparison of Torques at Joint 1 for Increasing Term Trigonometric Approximations. 


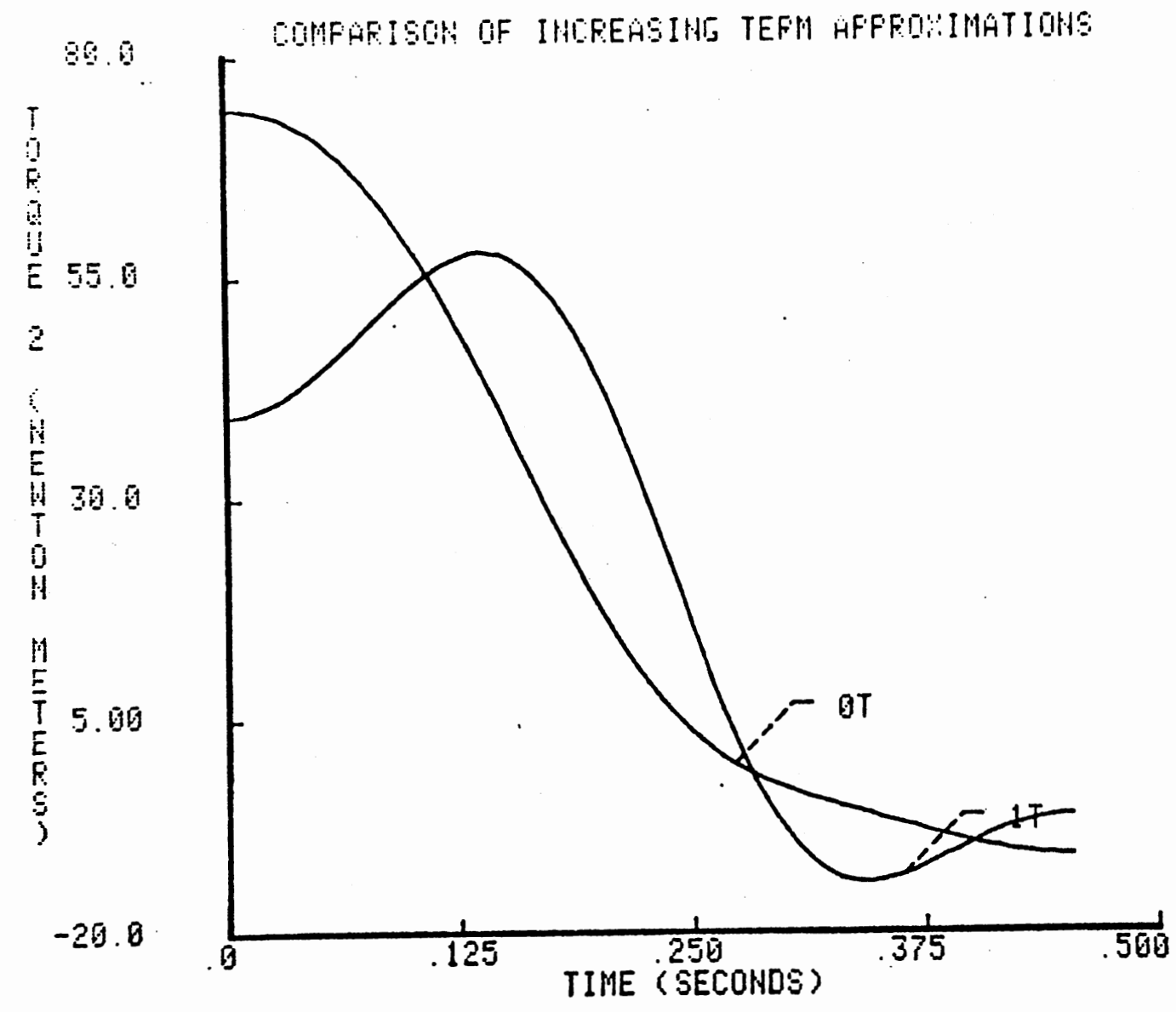

Figure 10. Comparison of Torques at Joint 2 for Increasing Term Trigonometric Approximations. 


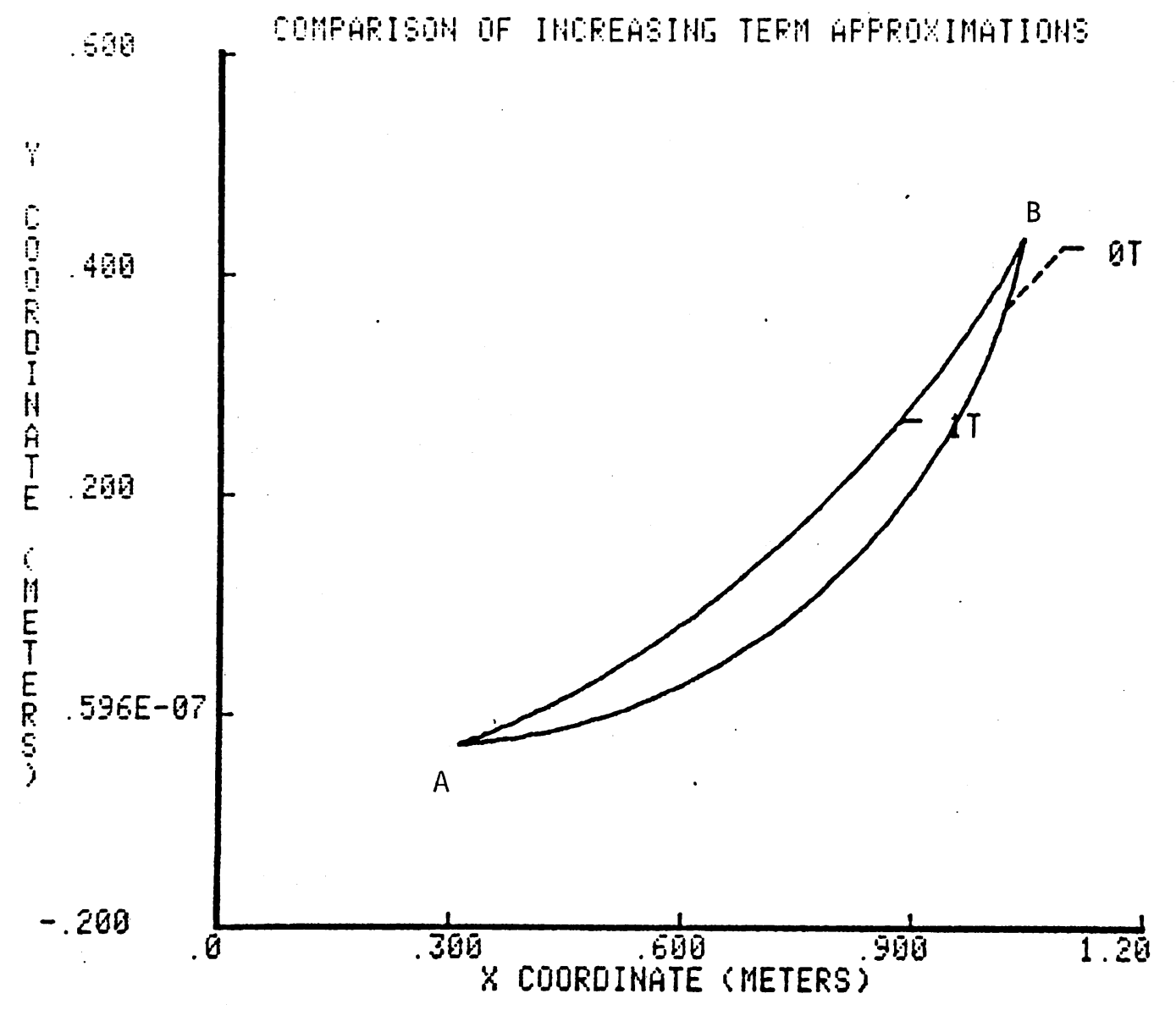

Figure 11. Comparison of End Effector Paths for Increasing Term Trigonometric Approximations 
applied to the lifting task at a traveling time of $T=1.0$ seconds. These results are shown in Figures 12 and 13 . Figure 12 shows that different joint motions occur for increasing terms in the approximating function. The objective function continually decreases in value for the increasing terms. Figure 13 demonstrates that adding one term is sufficient to approximate the optimal motion at joint two.

The conclusian to be drawn from the comparison of increasing term approximations at $T=0.45$ seconds and $T=1.0$ seconds is that no generalization should be made concerning the number of terms required. It is recommended that an approach as indicated in Equation (10) should be used in solving for the optimal joint motion for a specific task.

\subsection{Comparison of Polynomial and Trigono- metric Approximating Functions}

In choosing functions to be used in the Rayleigh-Ritz approximating function, it is required that increasing terms of the chosen functions should continually increase the accuracy of the approximation. Functions based on polynomials of increasing order and trigonometric terms of increasing harmonics meet this requirement. A comparison was made between the trigonometric approximations and polynomial approximations. The traveling time was fixed at $T=0.45$ seconds. Table 11 compares the weighting coefficients and the objective function for each case. Solutions were obtained for one term and two terms added to $\phi_{0}(t)$.

In examining Table 11 , it can be seen from the objective function values that the two approximations provide nearly the same results. The objective function values for the polynomial approximations are slightly better than those for the trigonometric approximation. It can also be 


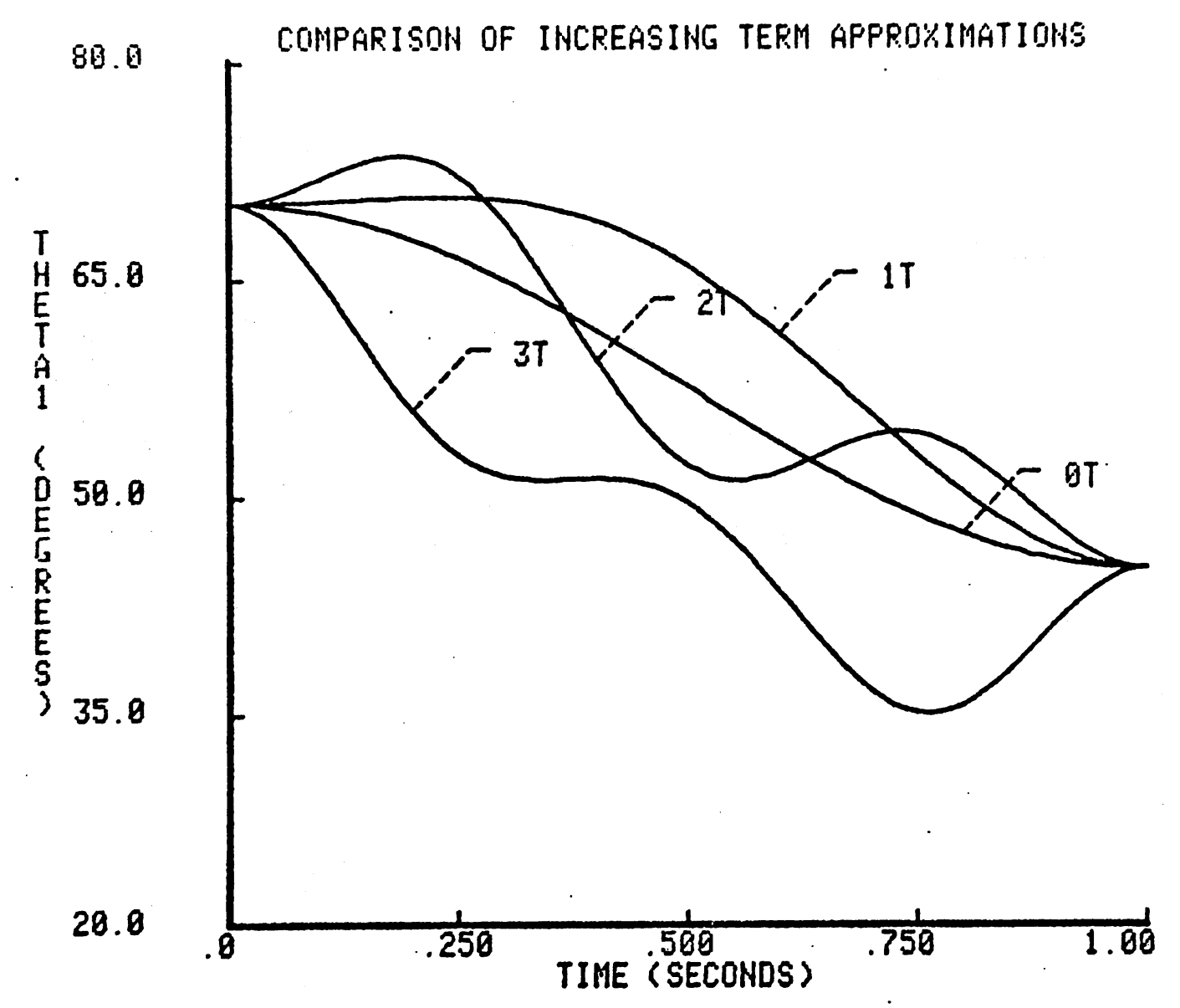

Figure 12. Comparison of Displacements at Joint 1 for $T=1.0$ Seconds. 


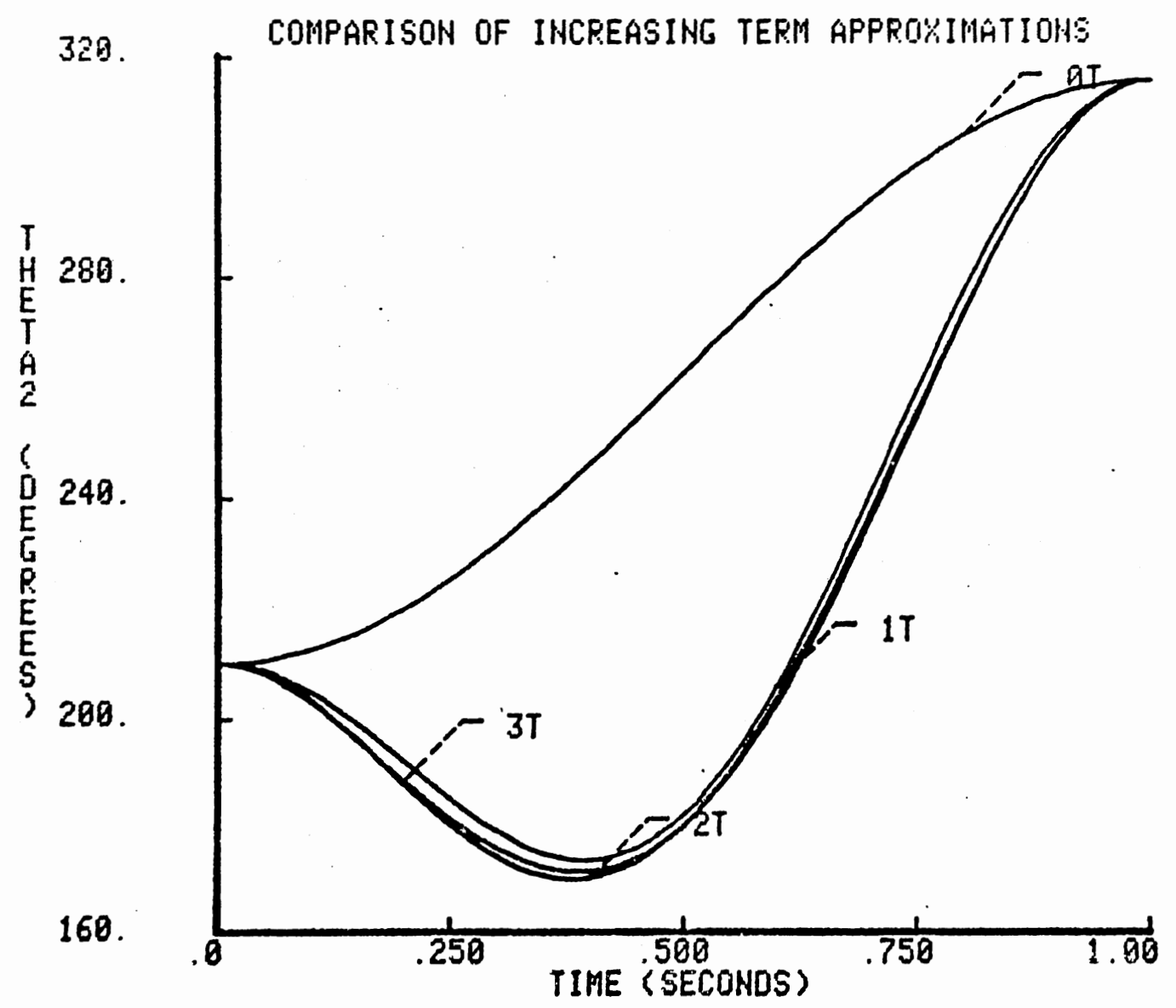

Figure 13. Comparison of Displacements at Joint 2 for $T=1.0$ Seconds. 
TABLE II

COMPARISON OF POLYNOMIAL AND TRIGONOMETRIC APPROXIMATING

FUNCTIIONS AT $T=0.45$ SECONDS

\begin{tabular}{|c|c|c|c|c|c|}
\hline $\begin{array}{l}\text { No. of } \\
\text { Terms }\end{array}$ & \multicolumn{2}{|c|}{ Starting Point } & \multicolumn{2}{|c|}{ Converged Point } & Objective \\
\hline \multicolumn{6}{|c|}{ Polynomial } \\
\hline 1 & $c_{11}=0.0$ & $c_{21}=0.0$ & $c_{11}=57.04$ & $c_{21}=-92.16$ & 1199.1 \\
\hline \multirow{2}{*}{2} & $c_{11}=0.0$ & $c_{21}=0.0$ & $c_{11}=56.29$ & $c_{21}=-94.45$ & \multirow{2}{*}{1197.4} \\
\hline & $c_{12}=0.0$ & $c_{22}=0.0$ & $c_{12}=6.25$ & $c_{22}=-2.42$ & \\
\hline \multicolumn{6}{|c|}{ Trigonometric } \\
\hline 1 & $c_{11}=0.0$ & $c_{21}=0.0$ & $c_{11}=0.0737$ & $c_{21}=-0.1165$ & 1220.0 \\
\hline \multirow{2}{*}{2} & $c_{11}=0.0$ & $c_{21}=0.0$ & $c_{11}=0.0742$ & $c_{21}=-0.1156$ & \multirow{2}{*}{1218.8} \\
\hline & $c_{12}=0.0$ & $c_{22}=0.0$ & $c_{12}=-0.000593$ & $c_{22}=-0.000223$ & \\
\hline
\end{tabular}


observed that no significant improvement is obtained in going from a one term polynomial to a two term polynomial. No meaningful comparison can be made between the weighting coefficients due to the different nature of the functions. Figures 14 and 15 compare the resulting joint motions. It can be clearly seen from these figures that similar results are obtained.

Trigonometric approximating functions were chosen for use in the remainder of the comparisons. This choice is based upon ease of use. The trigonometric terms are easier to formulate because the evaluation of angular velocity, $\dot{\theta}_{i}(t)$, and angular acceleration, $\ddot{\theta}_{i}(t)$, do not produce added terms in taking the derivatives, because the derivatives of sine and cosine terms produce only one term. For the polynomial functions having two time expressions, the derivative of each term produces two terms.

\subsection{Determination of Optimal Traveling Time, T}

The next factor considered was that of determining an optimal traveling time from station $A$ to station $B$. For this case, the traveling time $T$ becomes a variable in the optimization process. A one term trigonometric approximating function will be used. Caution must be exercised in determining the optimal traveling time due to the possible occurrence of local minima. Thus, multiple starting points must be used. The starting values of the weighting coefficients will still be chosen as $c_{11}=0$ and $c_{21}=0$. However, different starting values of $T$ will be chosen.

Table III summarizes the starting points used, the points converged to, and the value of the objective function at that point. In comparing the values of the objective function, it can be seen that the smallest local minimums occur at $T=0.454$ seconds $(I=1220.0)$ and $T=1.457$ seconds $(1=1192.0)$. Although the value of the objective function is lower 


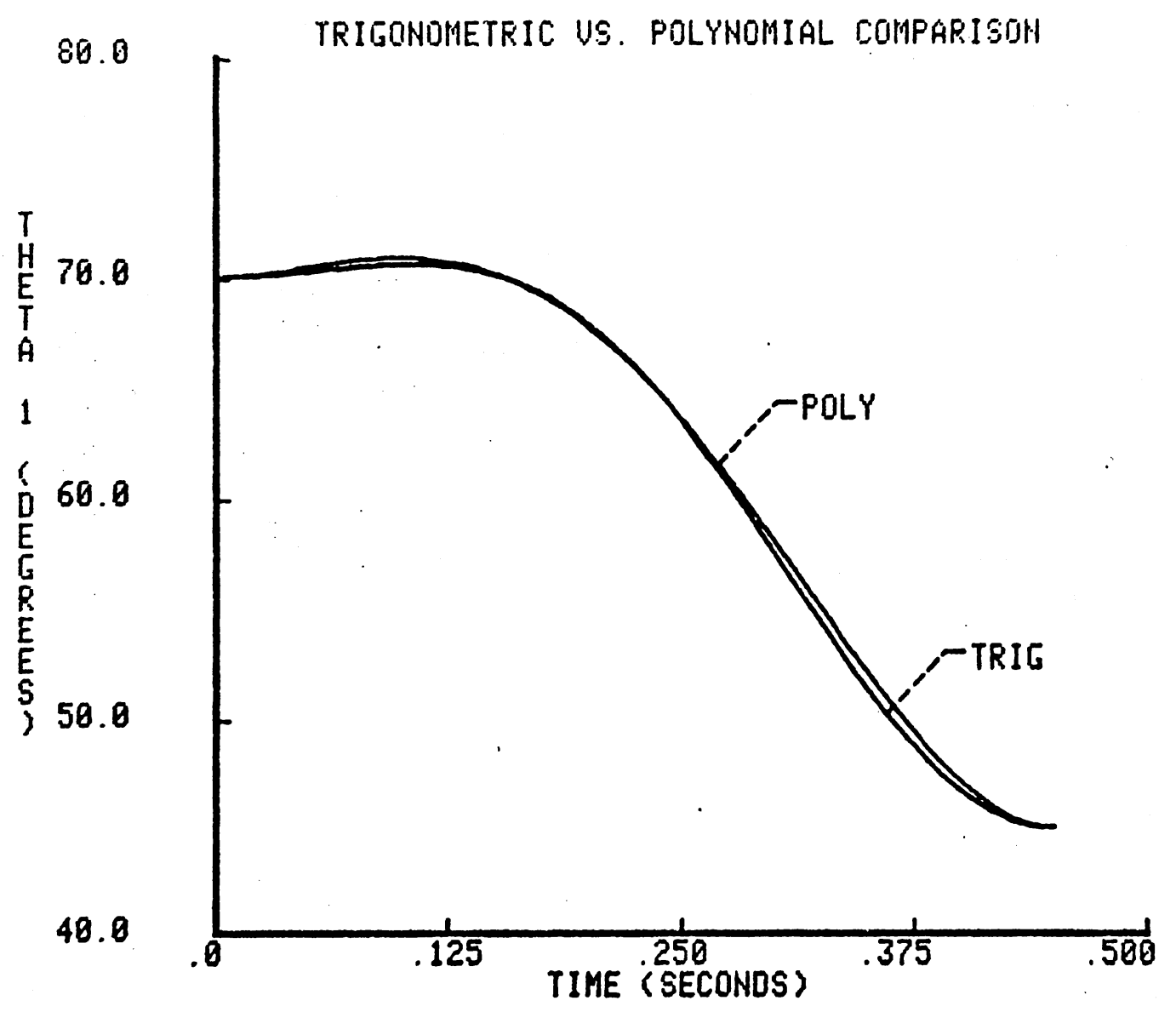

Figure 14. Comparison of Trigonometric and Polynomial Approximations of Displacement at Joint 1 . 


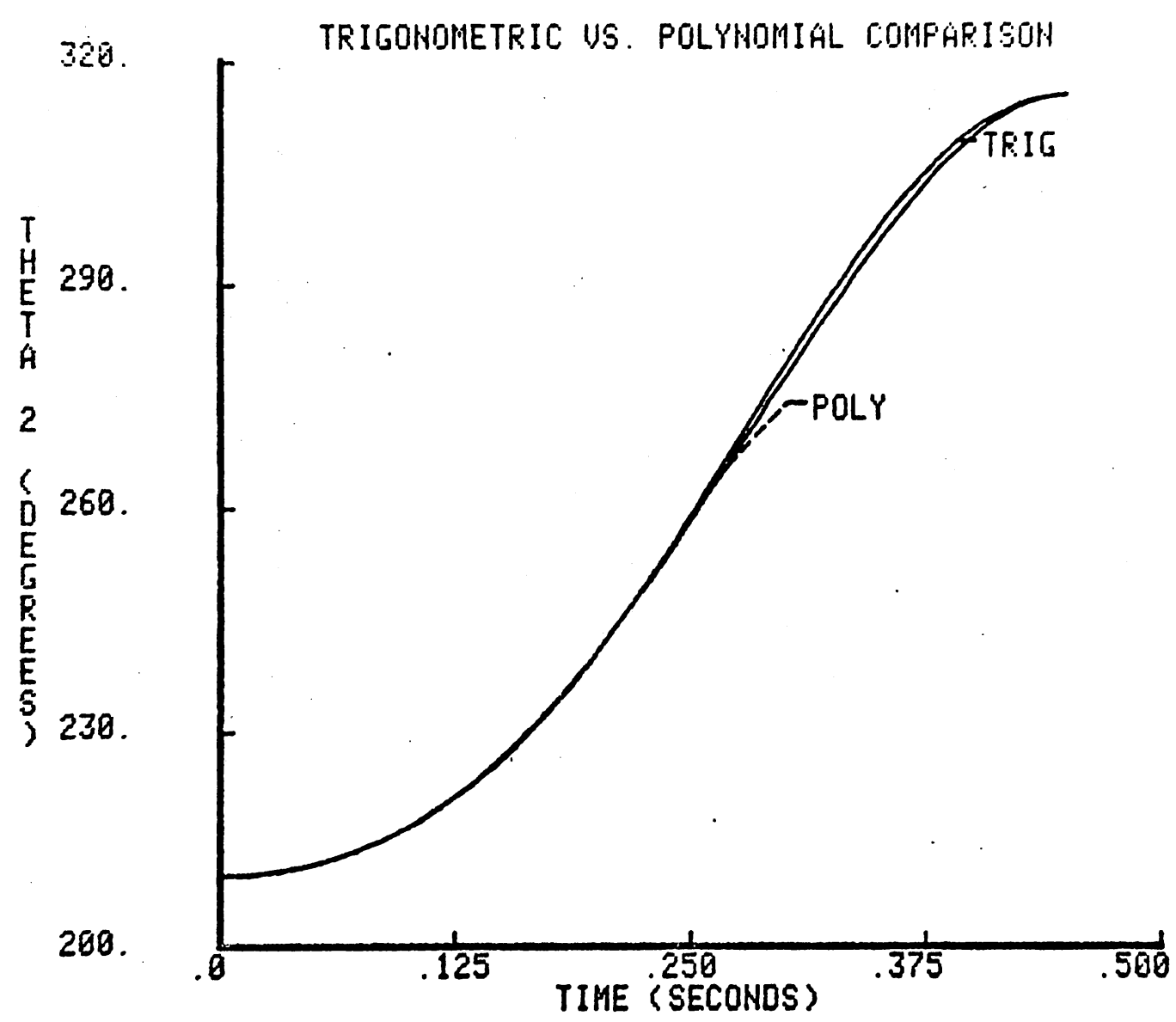

Figure 15. Comparison of Trigonometric and Polynomial Approximations of Displacement at Joint 2 
TABLE || $\mid$

DETERMINATION OF OPTIMAL TRAVELING TIME, T

\begin{tabular}{|c|c|c|}
\hline Starting Point & Converged Point & Objective \\
\hline$c_{11}=0.0 \quad c_{21}=0.0$ & $c_{11}=0.0736 \quad c_{21}=-0.1160$ & 1220.0 \\
\hline$T=0.1 \mathrm{sec}$ & $T=0.454 \mathrm{sec}$ & \\
\hline$c_{11}=0.0 \quad c_{21}=0.0$ & $c_{11}=0.0736 \quad c_{21}=-0.1160$ & 1220.0 \\
\hline$T=0.25 \mathrm{sec}$ & $T=0.454 \mathrm{sec}$ & \\
\hline$c_{11}=0.0 \quad c_{21}=0.0$ & $c_{11}=0.0737 \quad c_{21}=-0.1160$ & 1220.0 \\
\hline$T=0.5 \mathrm{sec}$ & $T=0.454 \mathrm{sec}$ & \\
\hline$c_{11}=0.0 \quad c_{21}=0.0$ & $c_{11}=-0.00471 \quad c_{21}=-0.8408$ & 1310.2 \\
\hline$T=1.0 \mathrm{sec}$ & $T=1.158 \mathrm{sec}$ & \\
\hline$c_{11}=0.0 \quad c_{21}=0.0$ & $c_{11}=-0.0309 \quad c_{21}=-1.1300$ & 1192.0 \\
\hline$T=1.5 \mathrm{sec}$ & $T=1.457$ & \\
\hline$c_{11}=0.0 \quad c_{21}=0.0$ & $c_{11}=0.2369 \quad c_{21}=-1.613$ & 1761.0 \\
\hline$T=2.0 \mathrm{sec}$ & $T=1.596$ & \\
\hline
\end{tabular}


at $T=1.456$ seconds, the traveling time chosen is $T=0.454$ seconds. For a very slight increase in the objective function value, the task can be completed three times faster.

An interesting factor is revealed in examining the end effector tra$i$ jectories at the two traveling times. Figure 16 shows the path for $T=$ 0.45 seconds. A regular, direct path is provided from A to B. Figure 17 shows the path for $T=1.46$ seconds. Notice that a direct path is not produced in this case. The end effector actually starts out in a direction away from $B$, and then turns around and takes a curved path to $B$. Thus, even though the motion program at $T=1.46$ seconds produces the lowest value for the objective function, it is not an acceptable motion program due to the end effector path. This motion program is discussed further in the next section.

\subsection{Comparison of Objective Functions}

Up to this point, the objective function used has been the integral over the traveling time of the sum of the square of the joint torques. However, other formulations of the objective function may also give satisfactory results. Possible objective functions include:
(1) minimize $1=\int_{0}^{T}\left(T_{1}^{2}+T_{2}^{2}\right) d t$
(2) $\operatorname{minimize} \quad 1=\int_{0}^{T}\left(\left|T_{1}\right|+\left|T_{2}\right|\right) d t$
(3) minimize $1=\operatorname{maximum}\left\{\left|T_{1}\right|,\left|T_{2}\right|\right\}$
(4) minimize $1=\int_{0}^{T}\left(T_{1} \dot{\theta}_{1}+T_{2} \dot{\theta}_{2}\right) d t$ 


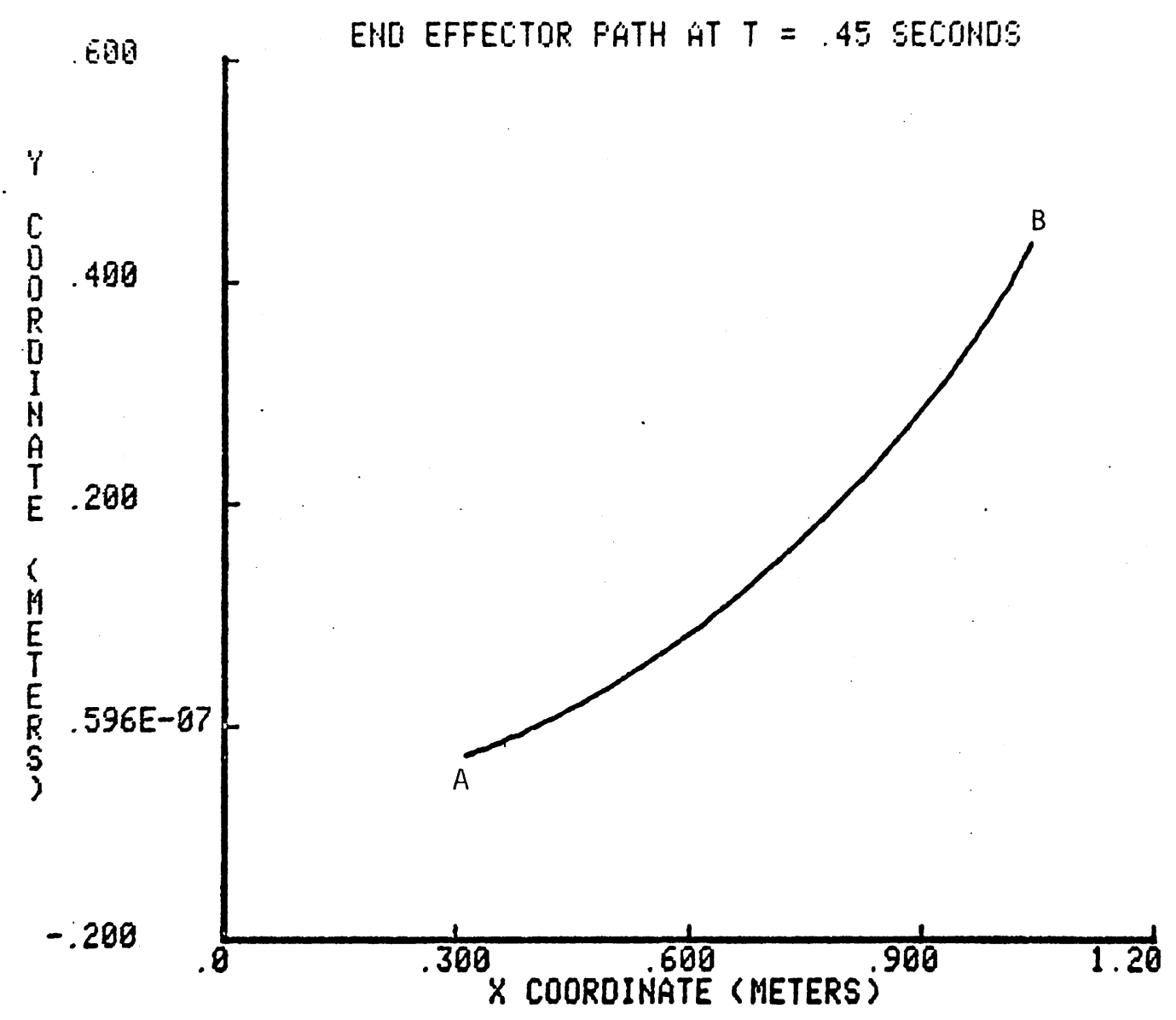

Figure 16. End Effector Path at $T=.45$ Seconds. 


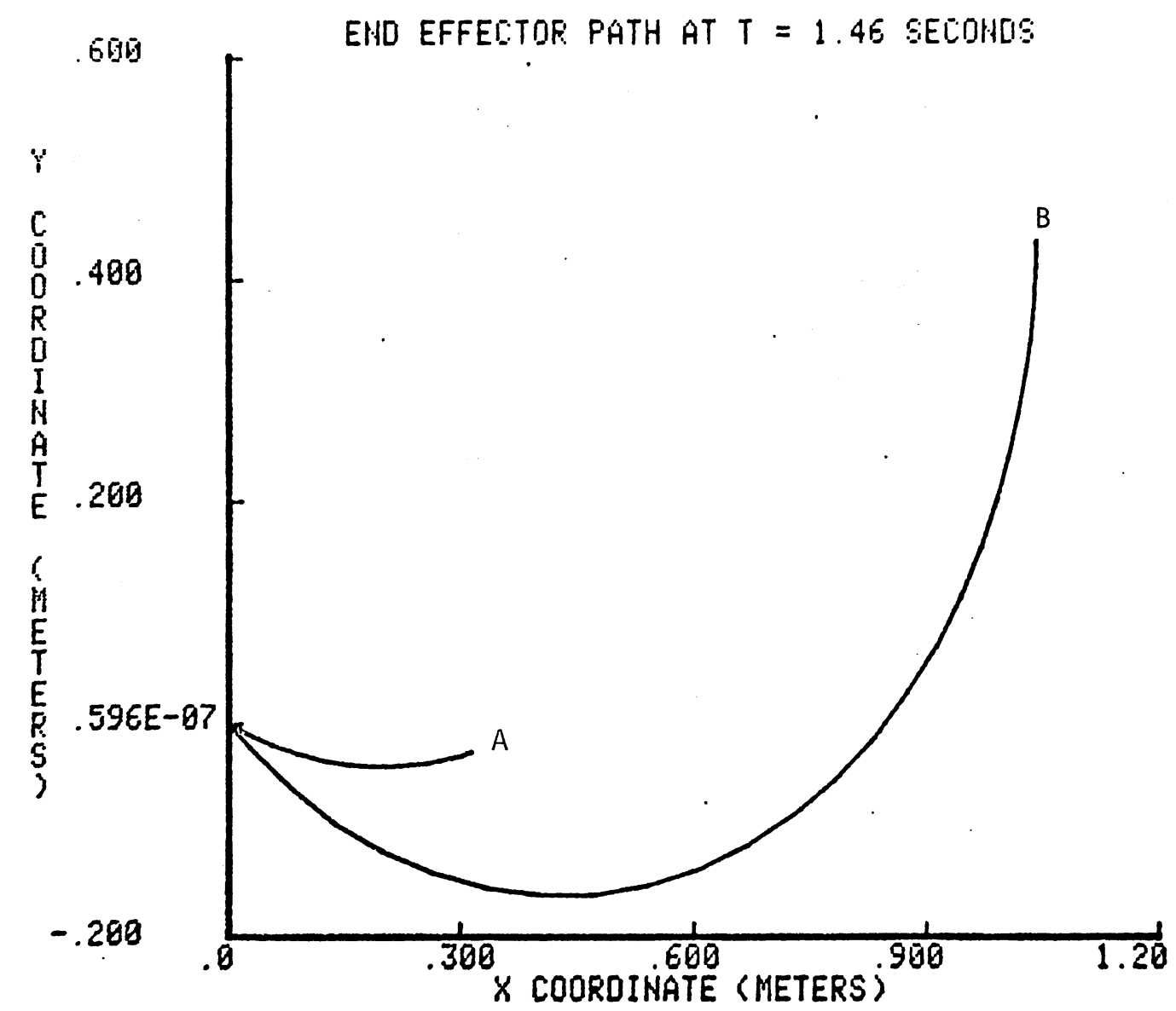

Figure 17. End Effector Path at $T=1.46$ Seconds. 
An examination of each of these objective functions will give some insight into the results which can be expected with their use. Traveling time $T$ is implicitly involved in all objective functions through its involvement in the joint torque expressions. Thus, the contribution to the value of the objective function by the torque expressions is effected by the traveling time. As the traveling time decreases, the joint torques become large because of the increasing magnitude of the joint velocities and accelerations. Traveling time is explicitly involved in objectives 1 and 2 because of the integration from 0 to $T$. Objective function 1 is more heavily weighted in terms of the joint torques than the traveling time. This is due to the squaring of each of the joint torque values. Objective function 2 provides an even weight between the joint torques and the traveling time. Objective function 3 minimizes the torque magnitude without involving the traveling time explicitly. Objective function 4 minimizes the energy consumed by the manipulator in traveling between positions $A$ and $B$. The mathematical model of the manipulator used in this thesis does not include the characteristics of the joint actuators. Thus, the system moves in a conservative force field and the energy consumed by the robot will be path independent. For this reason, objective function 4 will not be considered.

The objective functions were compared by performing a time optimization with a one term'trigonometric approximating function. The starting point value of $T$ was $T=0.5$ seconds. This is near the optimal value of $T$ indicated in the previous section. Table IV summarizes the results for this comparison.

Examination of Table IV shows that the different objective functions converge to different optimum traveling times. The joint displacement 
TABLE IV

COMPARISON OF OBJECTIVE FUNCTIONS

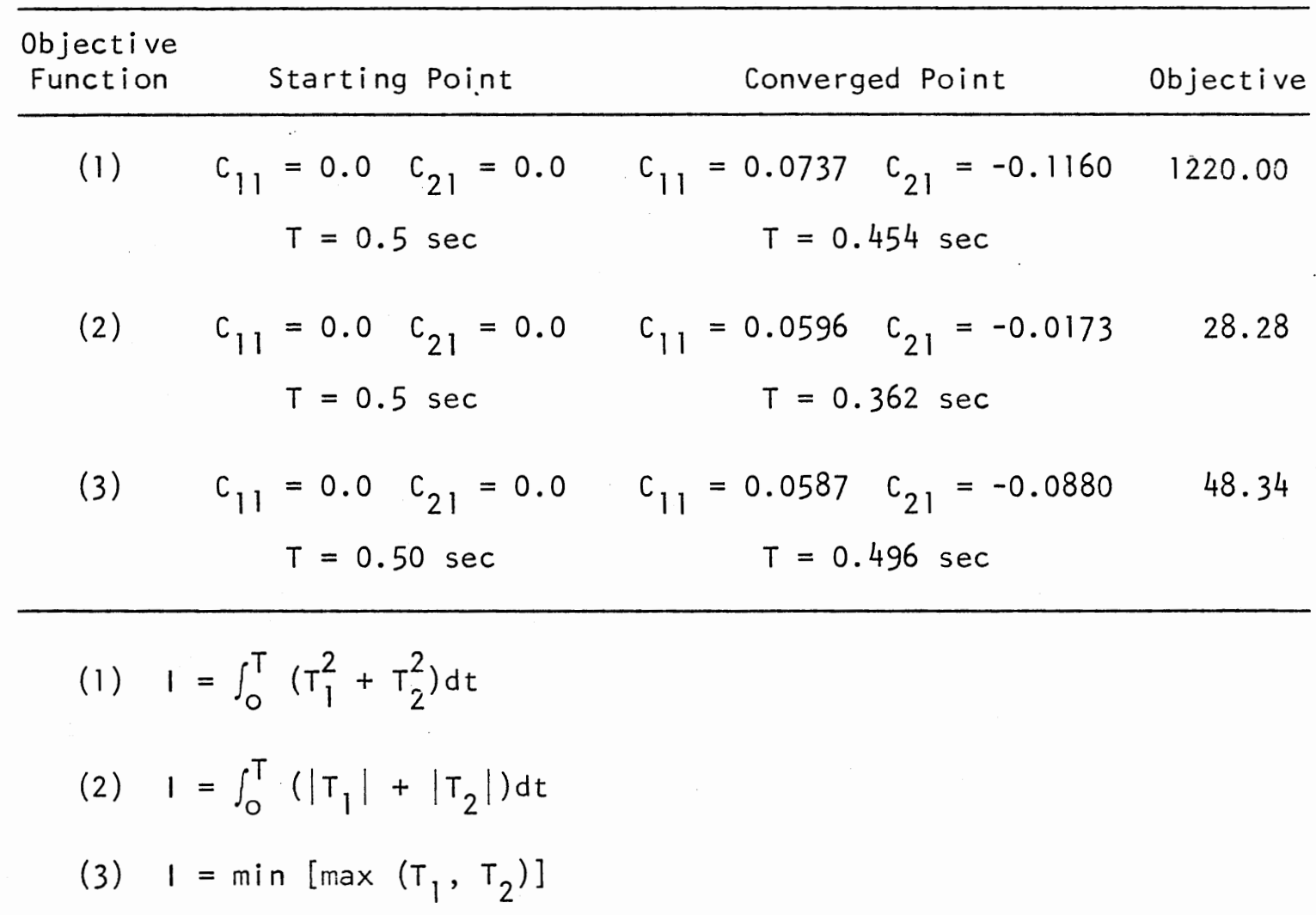


and torque profiles are shown in Figures 18 through 21 . The longest traveling time occurs for objective function 3. This is to be expected from the fact that $T$ is not explicitly involved in 3 , but only affects the objective function through its influence in the dynamics. Thus, it would tend to increase the traveling time from that found in 1 and 2 . However, examination of the joint torque profiles in Figures 20 and 21 shows that objective function 3 does indeed produce the lowest peak torques.

Objective function 2 produces the shortest traveling time of $\mathrm{T}=$ 0.362 seconds. This objective function has a more even weight between the torque values and the traveling time than that of 1 . Thus, the peak torque values are sacrificed for a smaller value of $T$. However, examination of the torque profiles in Figures 20 and 21 indicate that the results are unacceptable. The objective function produces large positive and negative joint torque peaks.

Objective function 1 tends to produce the best results. It provides a traveling time of 0.45 seconds. The joint torque profiles are very similar to those of objective 3 , while still maintaining a shorter traveling time. Although $T$ is greater than that for objective function 2 , the joint torque profiles are much more acceptable for objective function 1 . Figure 22 compares the end effector path for the three objective functions. It can be seen that all of the objective functions produce a regular, direct path from station A to station B.

From this comparison, it can be concluded that objective functions 1 and 3 provide acceptable results. Objective function 1 tends to produce a shorter traveling time than that of 3 , with a slight increase in the peak value of torques. Both objective functions provide a direct path 


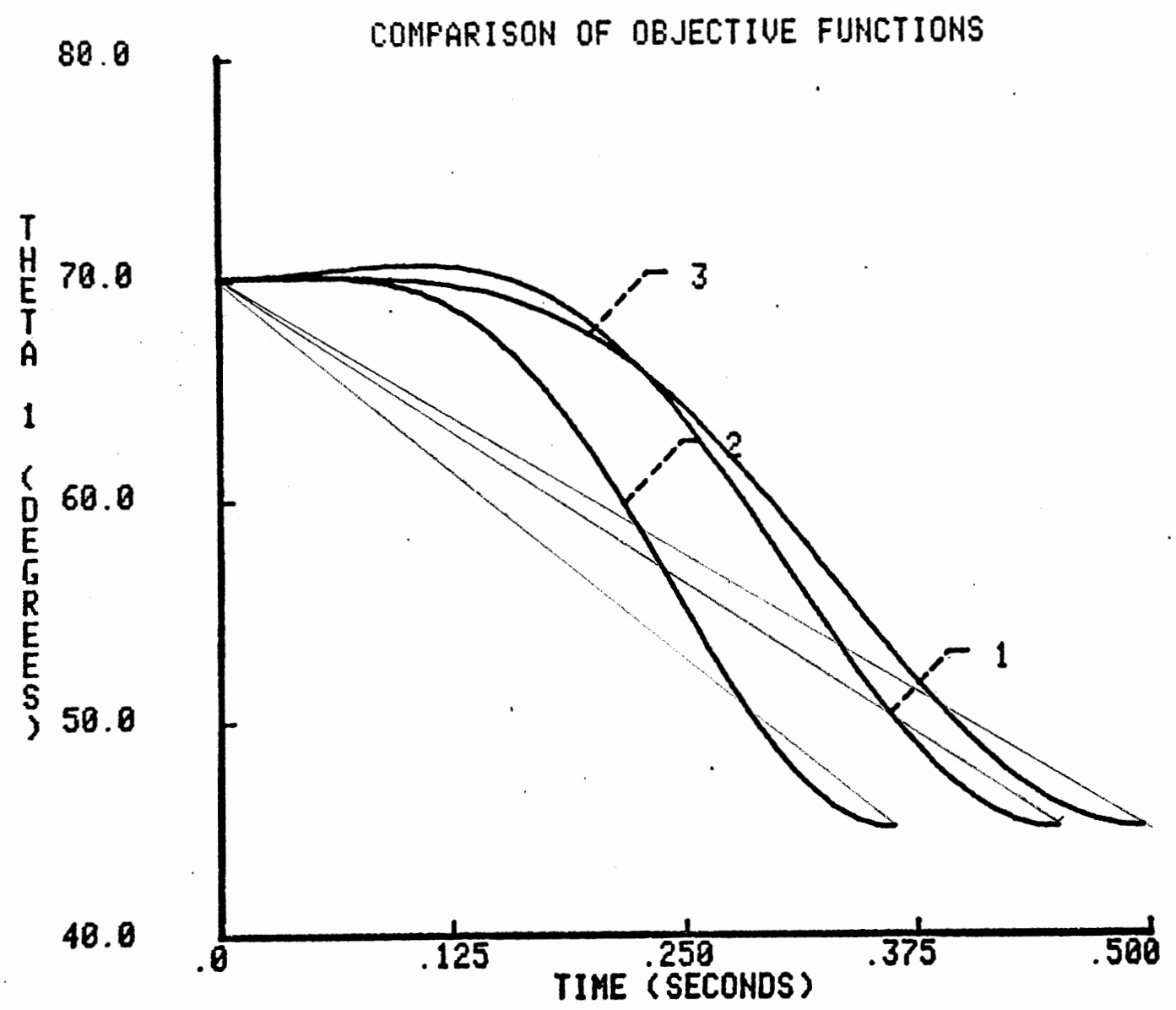

Figure 18. Comparison of Displacements at Joint 1 for Various Objective Functions. 


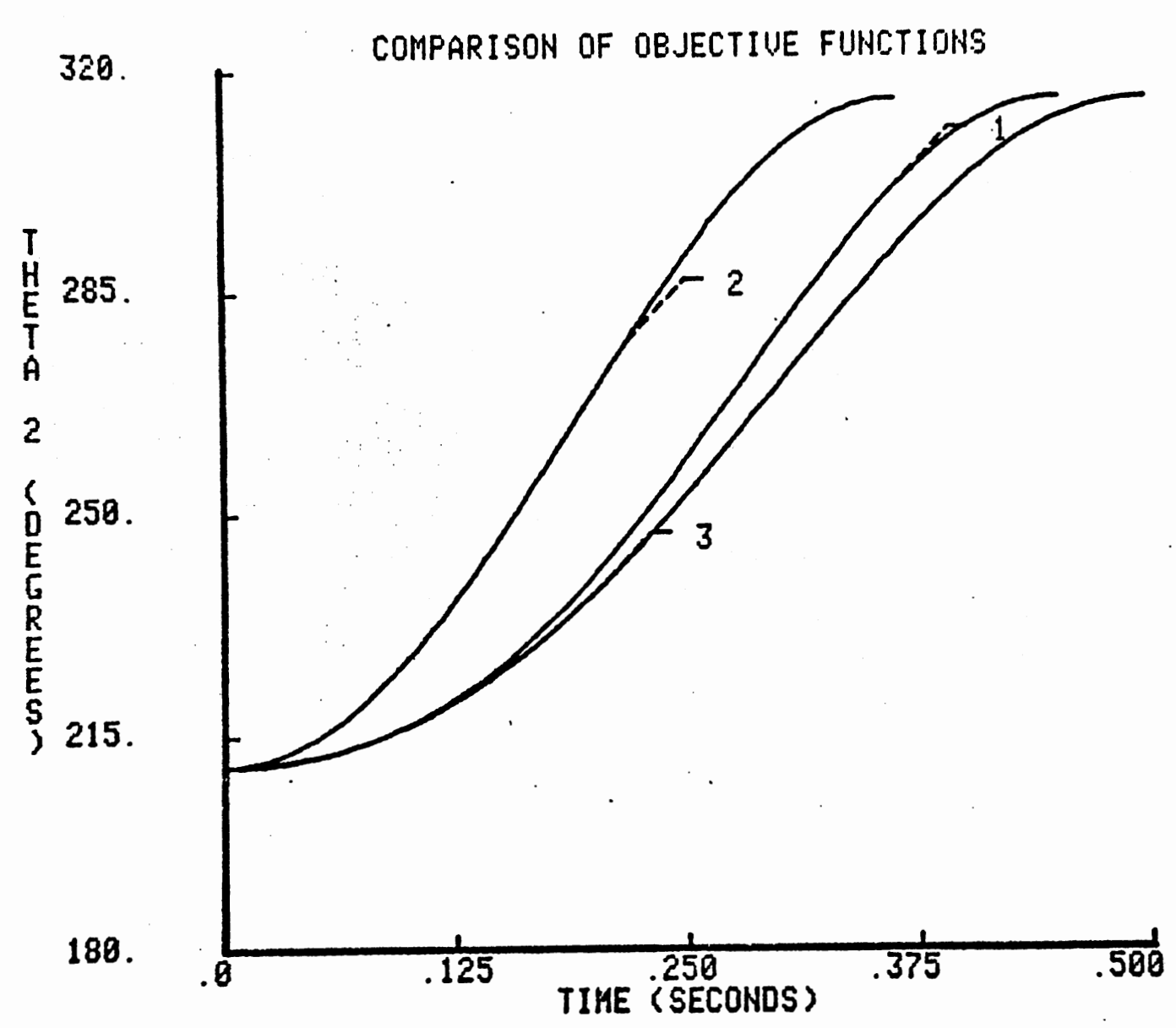

Figure 19. Comparison of Displacements at Joint 2 for Various objective Functions. 


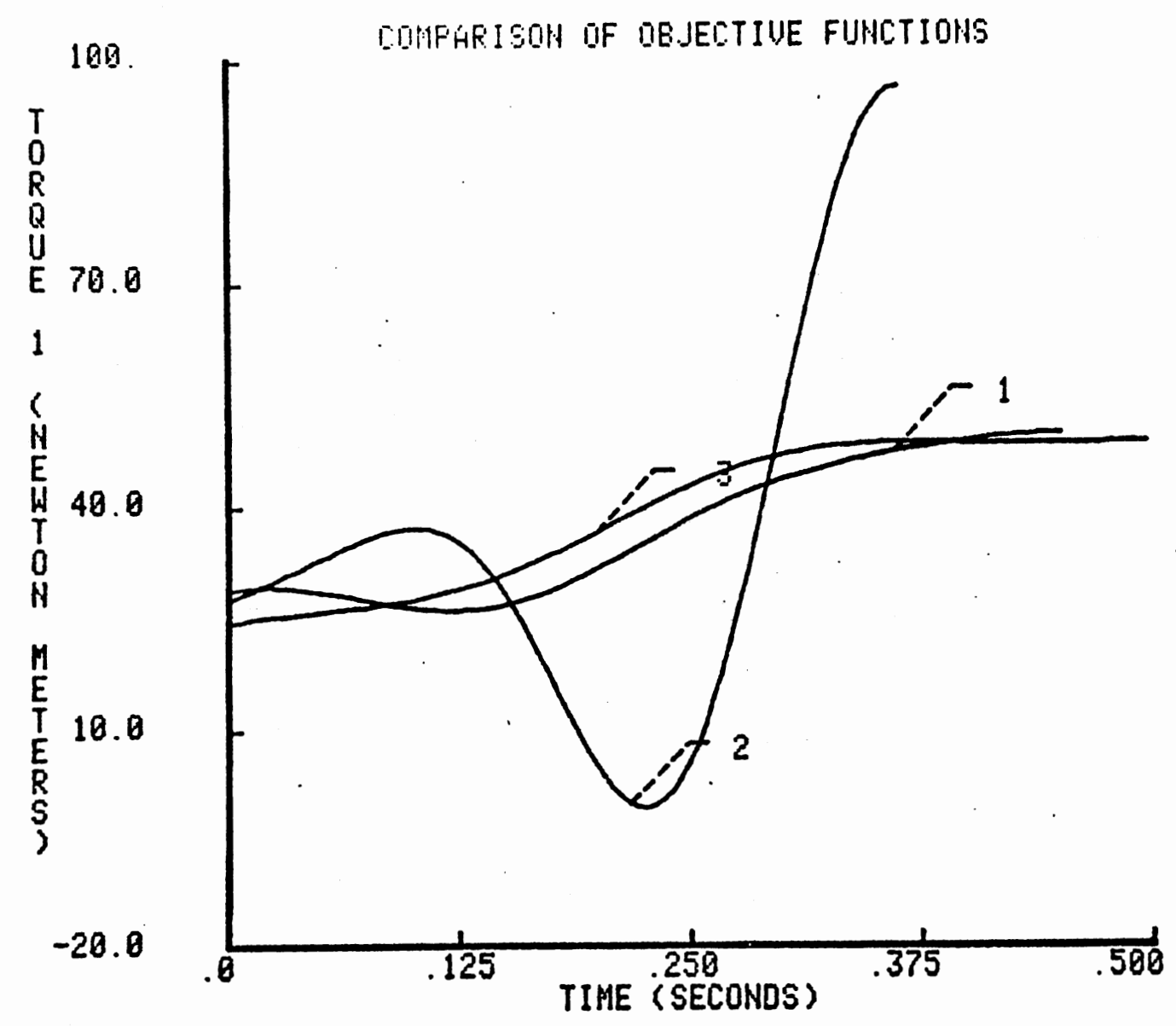

Figure 20. Comparison of Torques at Joint 1 for Various objective Functions. 


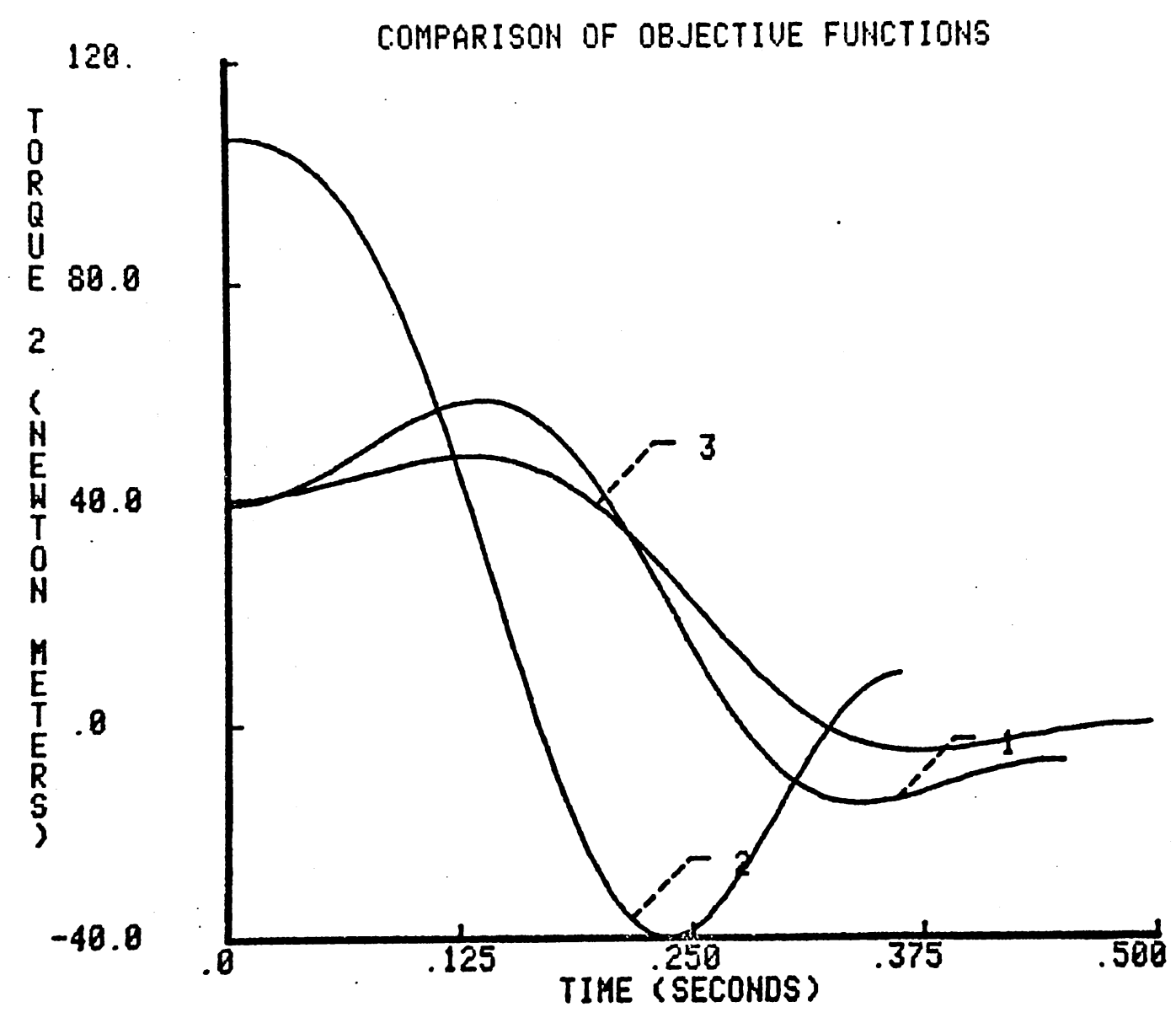

Figure 21. Comparison of Torques at Joint 2 for Various objective Functions. 


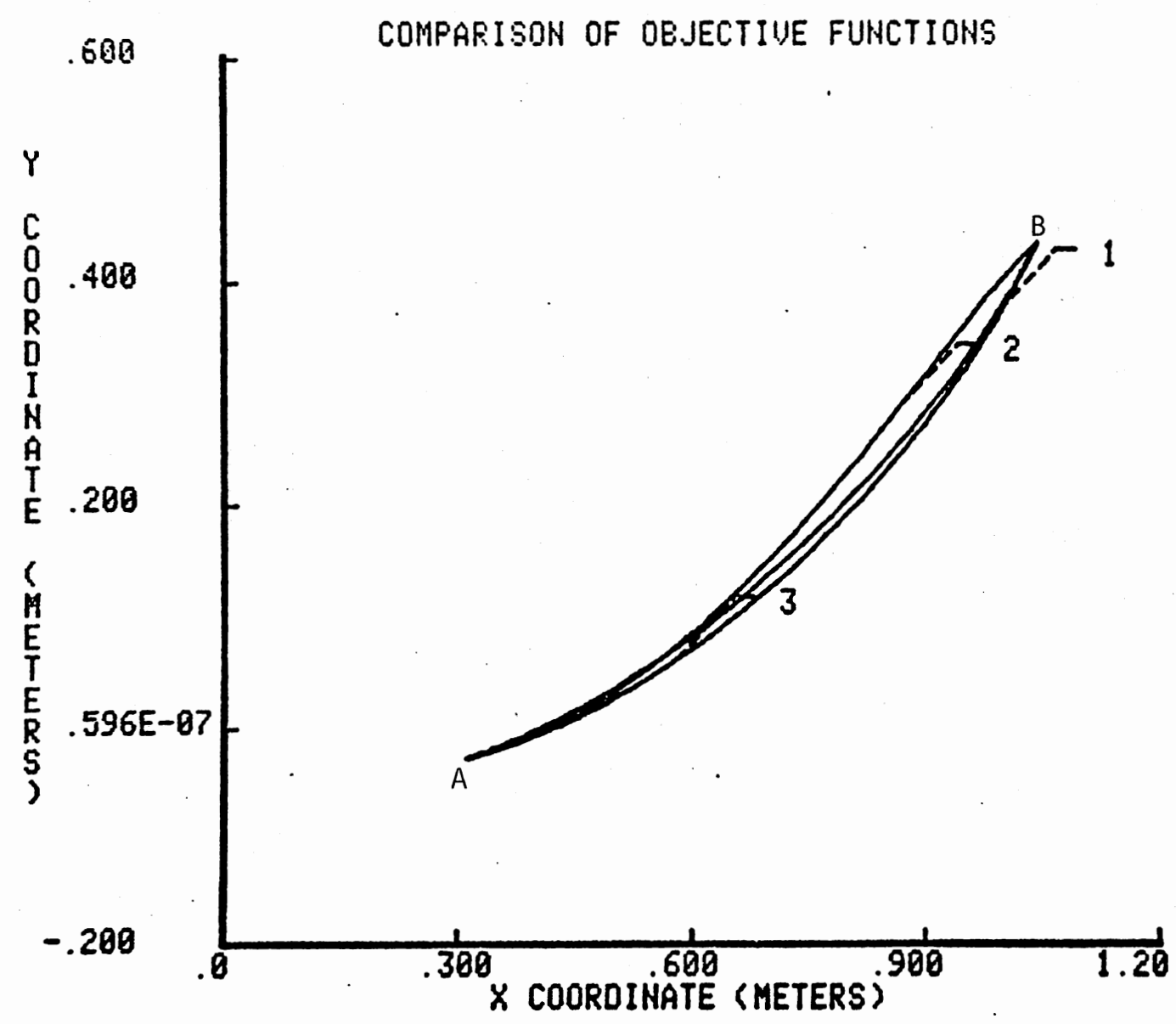

Figure 22. Comparison of End Effector Paths for Various objective Functions. 
from $A$ to $B$. However, it should be noted that these objective functions are being compared at their optimal traveling times.

In the previous section, optimal traveling times were indicated at $T=1.457$ seconds and $T=0.454$ seconds. The shorter traveling time was chosen not only because it completed the task three times faster, but it also provided a direct path from A to B (see Figures 16 and 17). However, suppose the traveling time were constrained to a given value. Is it possible to obtain an acceptable optimal joint motion program?

Objective function 1 was used in determining the optimal traveling time. The joint torque profiles at $T=1.4565$ seconds are shown in Figures 23 and 24 . It can be seen that the optimal value of 1 is obtained by having the torque fluctuate about zero for a substantial amount of the traveling time, and then having rapid changes in torque near the end of the time period in order to reach the final destination. The resulting motion of the second link of the robot is shown in Figure 25 . Figure 25 is a plot of $\theta_{1}(t)+\theta_{2}(t)$ versus time which gives the orientation of the second link with respect to the horizontal. The initial position of the link is $\theta_{1}(0)+\theta_{2}(0)=280.0^{\circ}$, the desired final position is $\theta_{1}(T)+$ $\theta_{2}(T)=360.0^{\circ}$. It can be seen that during the initial motion of the link, it actually moves to a position $180.0^{\circ}$ away from its desired final position. Only during the last 0.5 seconds does the second link make any progress toward its final destination. This sporadic motion of the second link explains the undesirable end effector motion in Figure 17.

In hopes of finding acceptable joint motion programs, objective function 3 was applied at $T=1.456$ seconds. Figures 26 and 27 compare the resulting torque profiles. It can be seen that objective function 3 produces a smooth torque profile at both joints. The peak torque value is 


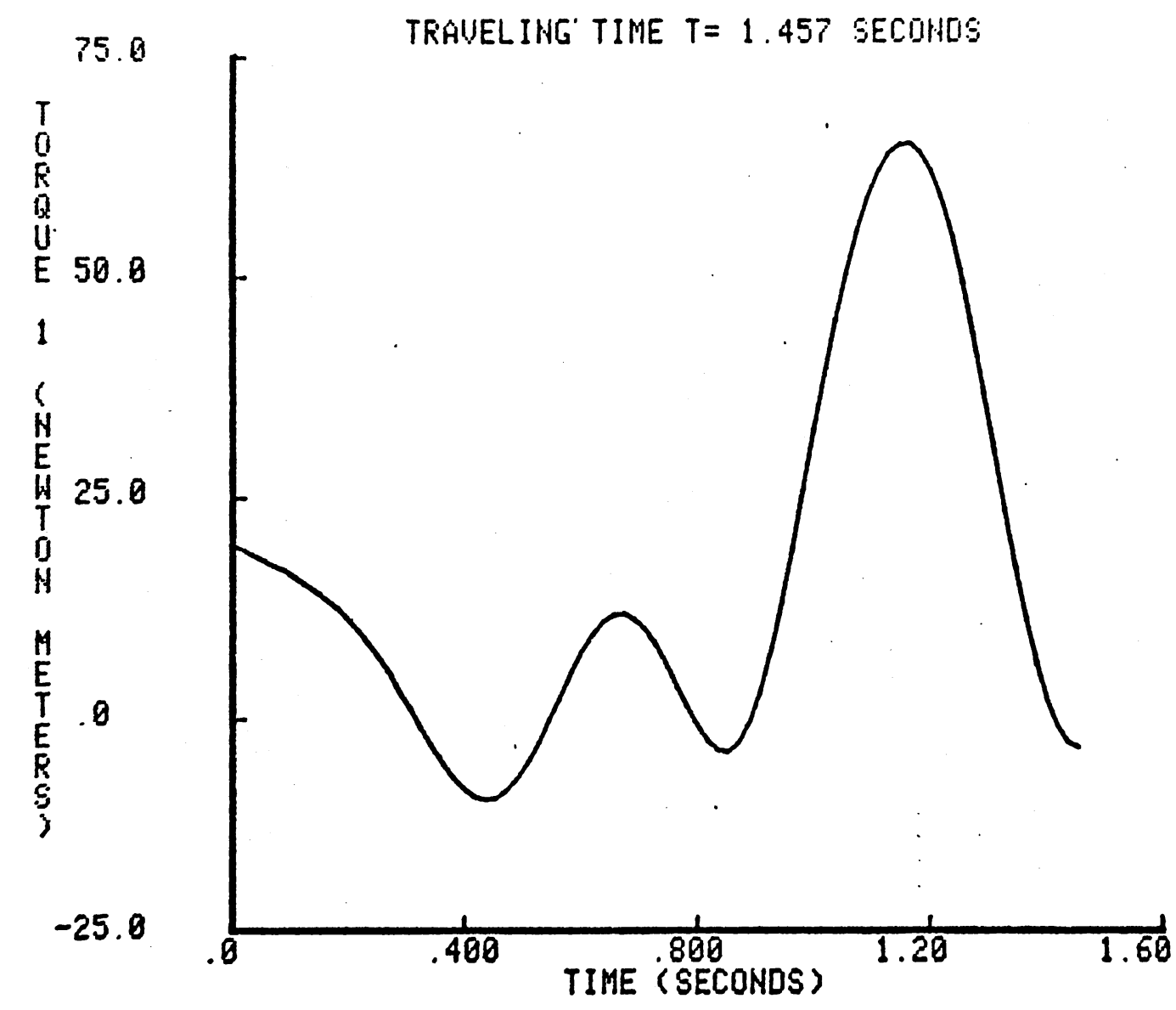

Figure 23. Torque at Joint 1 for $T=1.457$ Seconds. 


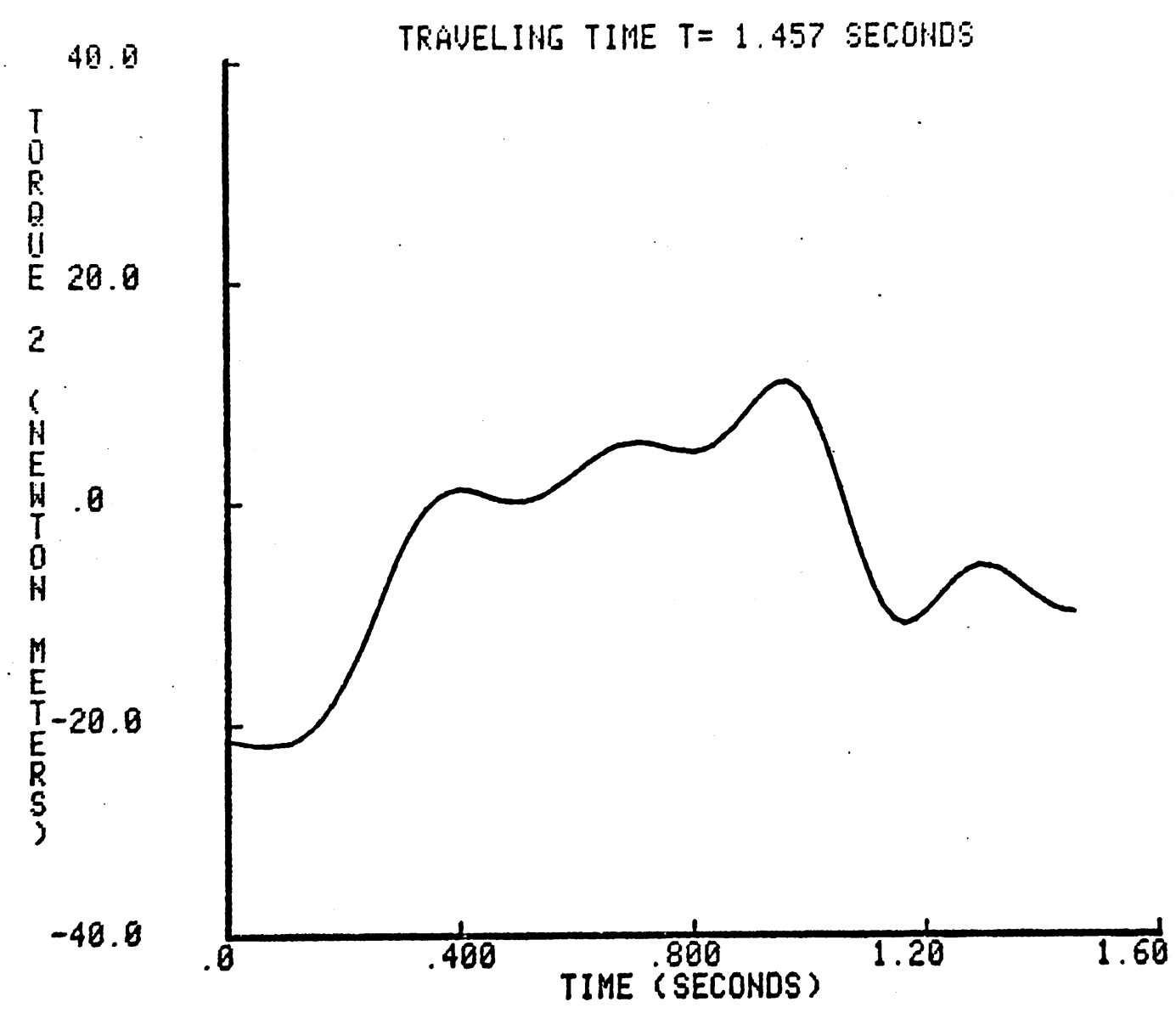

Figure 24. Torque at Joint 2 for $T=1.457$ Seconds. 


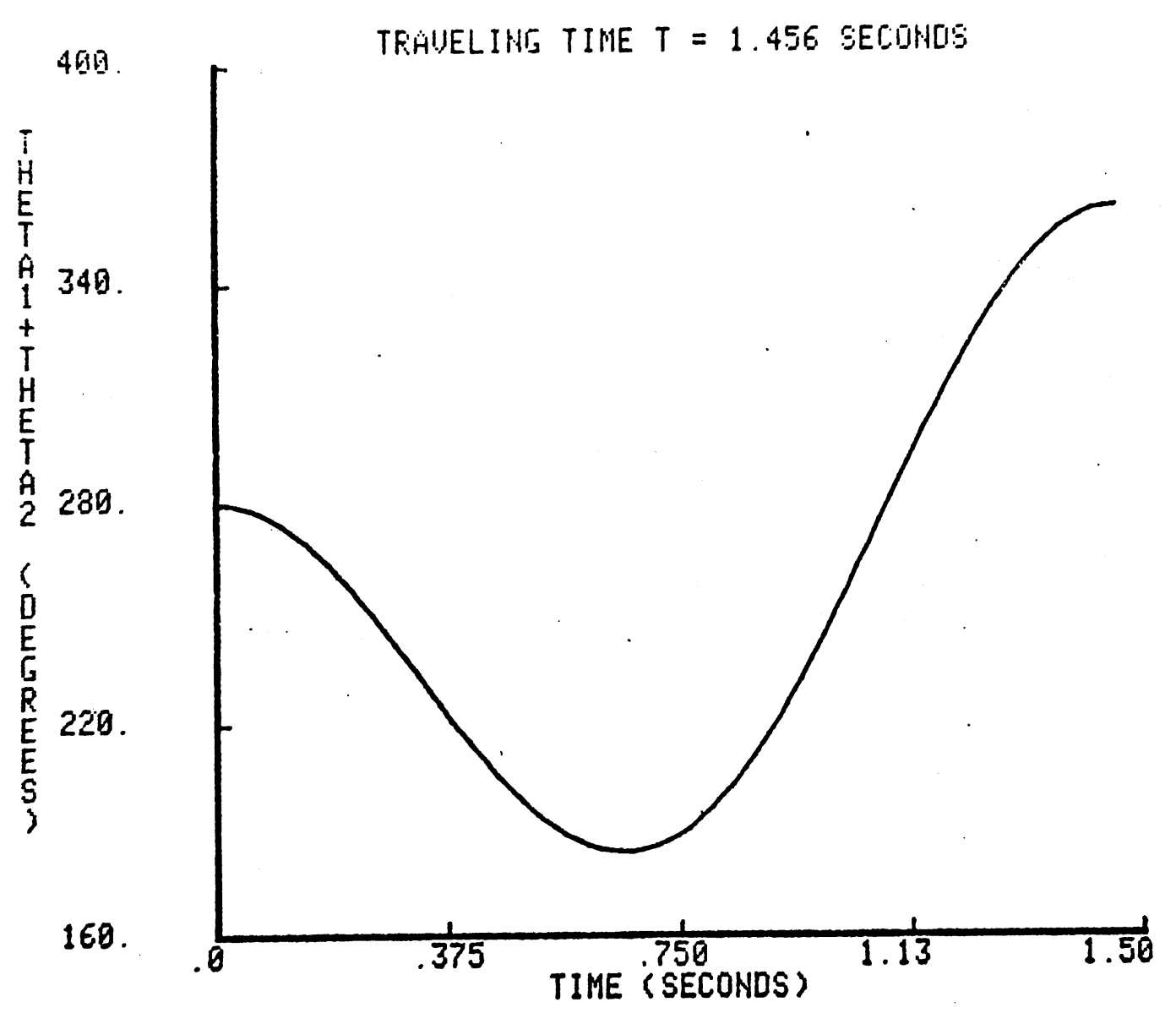

Figure 25. Orientation of Link 2 for $T=1.457$ Seconds. 


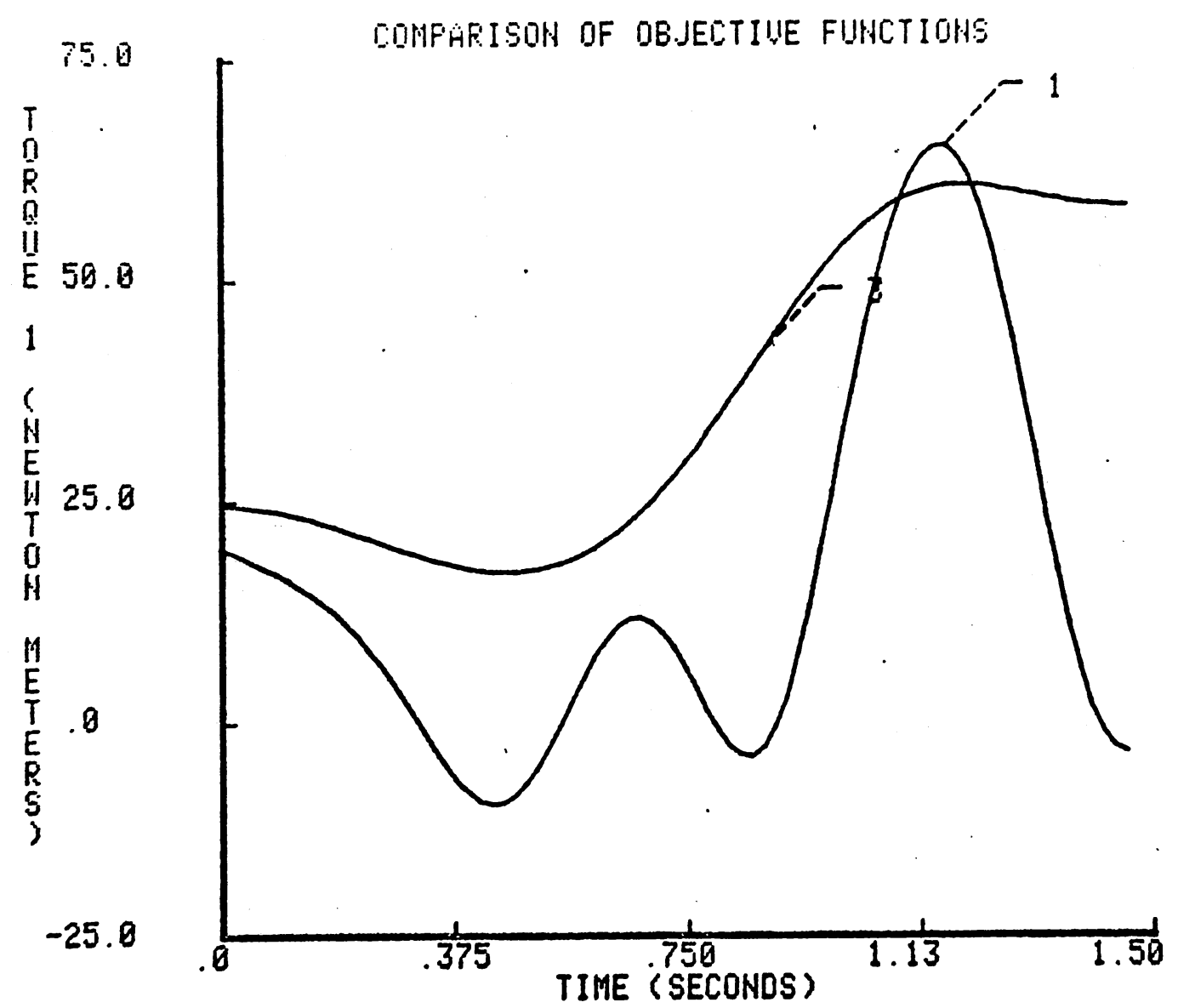

Figure 26. Torques at Joint 1 for Objective Functions 1 and 3 . 


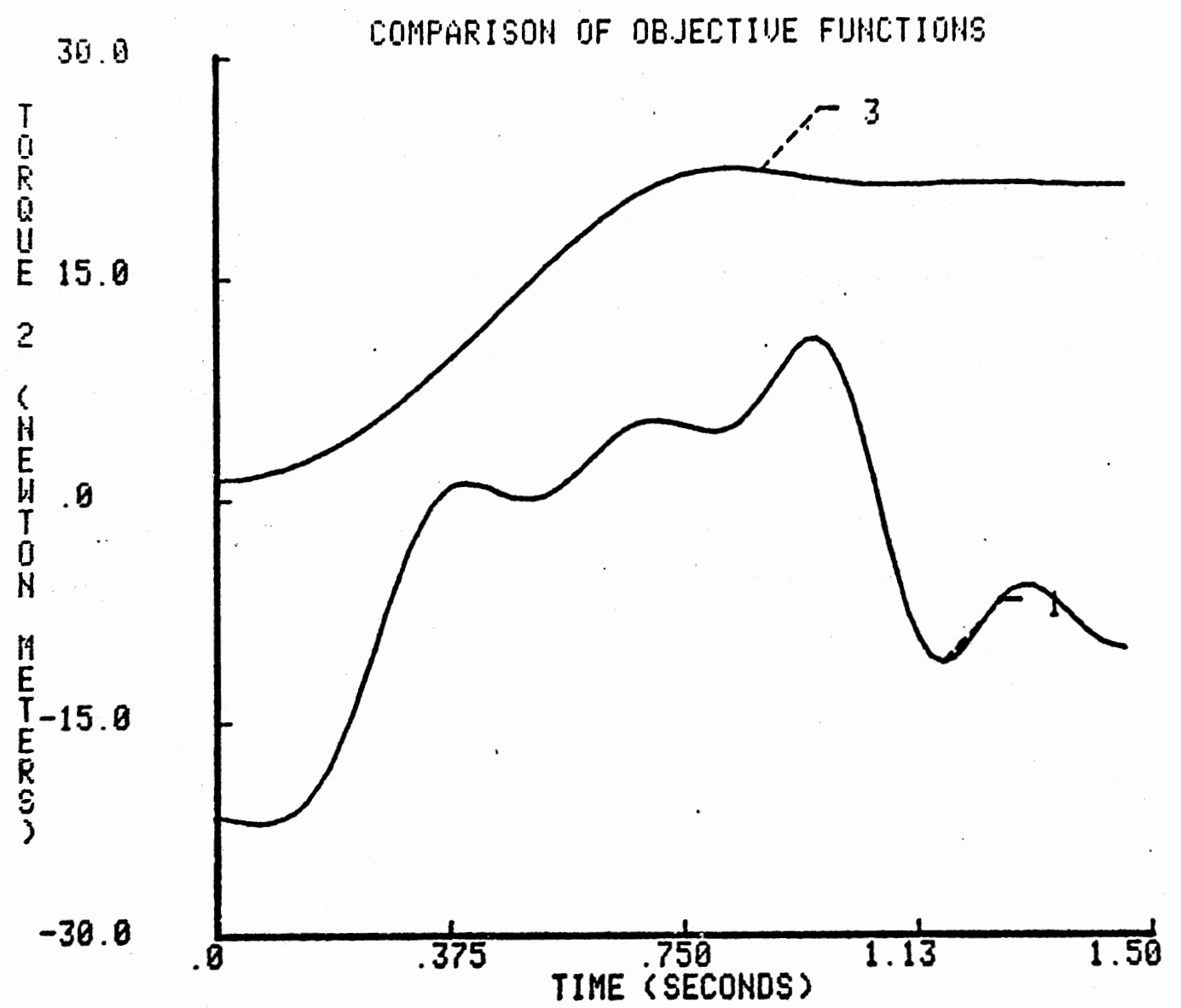

Figure 27. Torques at Joint 2 for Objective Functions 1 and 3 . 
slightly reduced at joint one, and of nearly equal magnitude at joint two. The true test is the resulting end effector trajectory shown in Figure 28. It can be seen that objective function 3 provides a much better end effector path from $A$ to $B$. During the initial motion, the end effector starts in a direction away from $B$, but quickly corrects itself and moves along a direct path.

Thus, by using objective function 3 , an opt imal motion program can be obtained which provides an acceptable end effector path, as well as smooth joint torque profiles. The conclusion from all of these comparisons is that objective function 1 should be applied when optimization of traveling time is the major concern, and objective function 3 when optimal joint motions are required at a fixed traveling time. Also, it has been shown that objectives 1 and 3 give similar results at a fixed value of $T$ if $T$ is the optimal traveling time.

\subsection{Comparison of Optimal and Standard Solutions}

The result of the comparisons in the past sections indicate an optimal motion program exists at $T=0.45$ seconds. The optimal motion is approximated by one term trigonometric functions with weighting coefficients of $C_{11}=0.0737$ and $c_{21}=-0.1165$. In this section, the optimal motion will be compared to a standard motion used in industry. The standard motion consists of specifying a straight line end effector path between station $A$ and station $B$, and specifying that the motion along the path be that of a modified trapezoidal motion program [28]. The displacement $s$, velocity $\dot{s}$, and acceleration $\ddot{s}$, characteristics of the modified trapezoidal motion program are shown in Figure 29. 


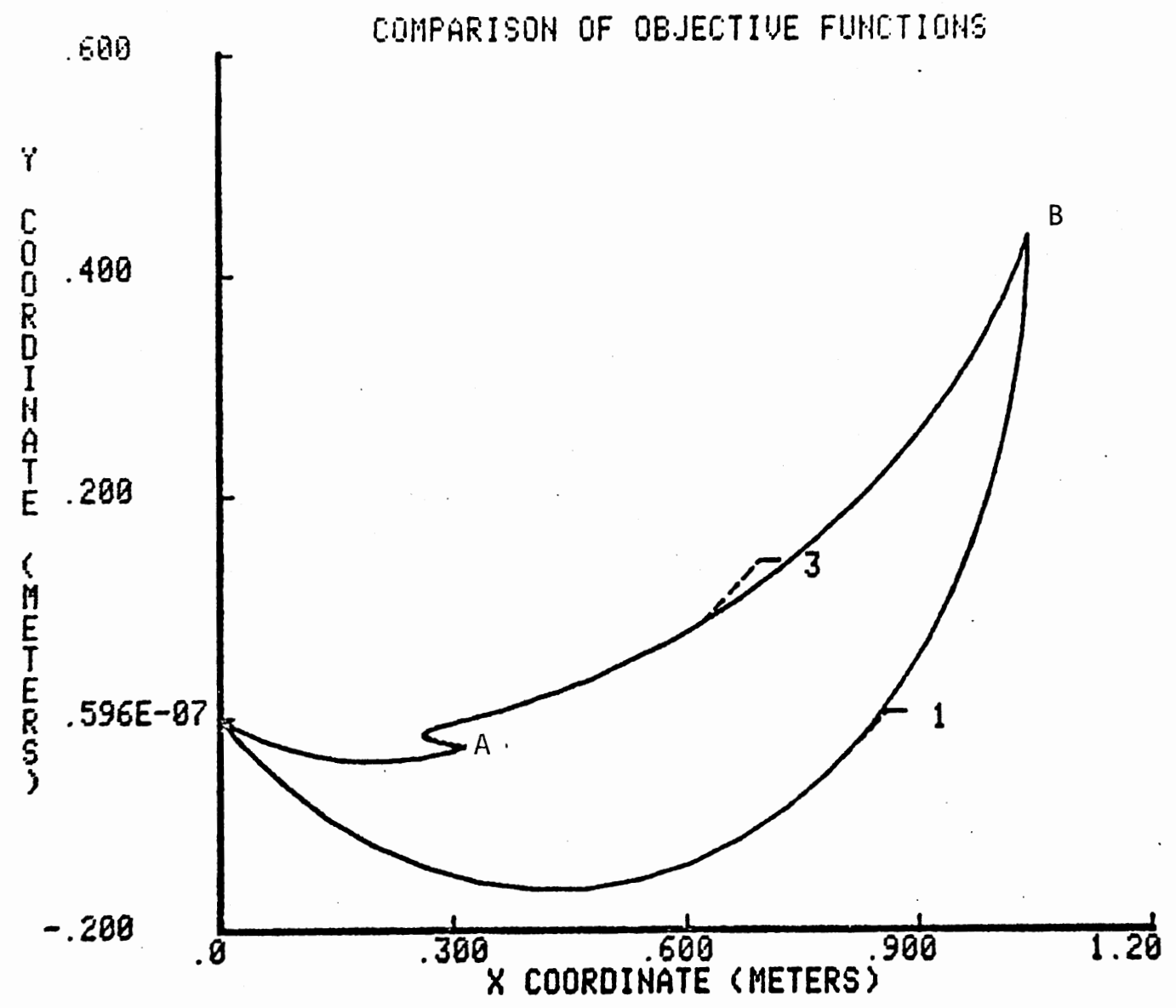

Figure 28. End Effector Paths for Objective Functions 1 and 3. 


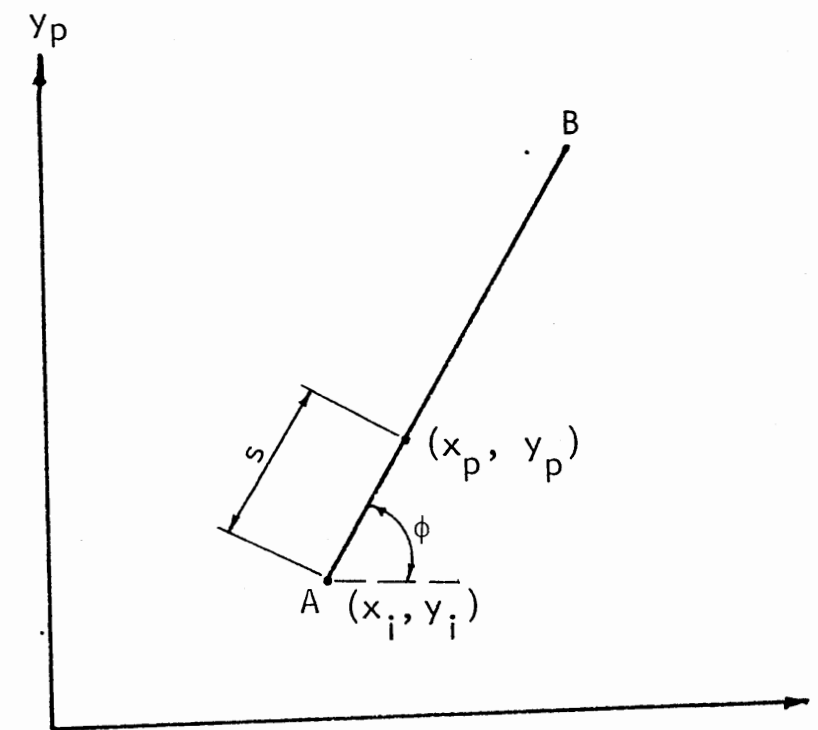

End Effector Path

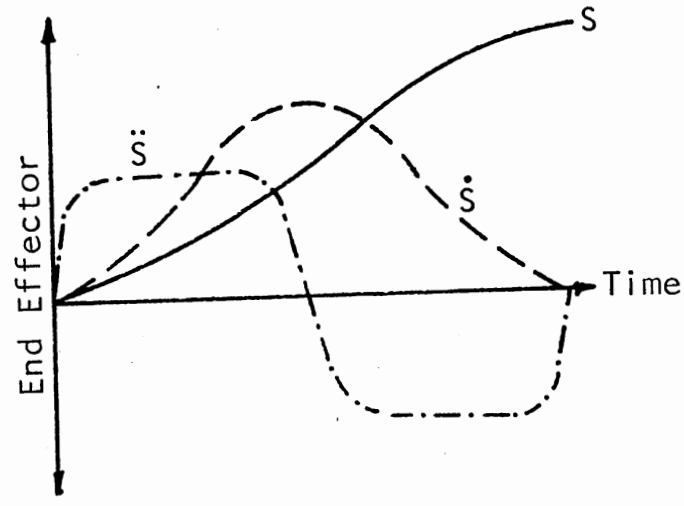

Modified Trapezodial Motion Program

Figure 29. Standard End Effector Path and Motion Program. 
An inverse problem must be solved in order to determine the kinematic and dynamic parameters of the manipulator so the objective function in Equation (1) can be evaluated. That is, the position, velocity and acceleration of the end effector (point $P$ in Figure 5) are specified by the modified trapezoidal motion program, and it is desired to determine the angular. displacement, velocity and acceleration expressions for each joint of the robot. Examination of Equations (2) through (7) shows that the parameters on the left-hand side are known and can be expressed as foll lows:

$$
\begin{aligned}
& x_{p}=x_{i}+s \cos \phi \\
& y_{p}=y_{i}+s \sin \phi \\
& \dot{x}_{p}=\dot{s} \cos \phi \\
& \dot{y}_{p}=\dot{s} \sin \phi \\
& \ddot{x}_{p}=\ddot{s} \cos \phi \\
& \ddot{y}_{p}=\ddot{s} \sin \phi
\end{aligned}
$$

The parameters on the right-hand side, $\theta_{i}, \dot{\theta}_{i}, \ddot{\theta}_{i}$, are to be determined. Equations (2) through (7) can be rewritten in the following form:

$$
\begin{aligned}
& {\left[\begin{array}{c}
\Delta x_{p} \\
\Delta y_{p}
\end{array}\right]=[\mathrm{J}]\left[\begin{array}{l}
\Delta \theta_{1} \\
\Delta \theta_{2}
\end{array}\right\}} \\
& \left\{\begin{array}{c}
\dot{x}_{p} \\
\dot{y}
\end{array}\right\}=[\mathrm{J}]\left\{\begin{array}{l}
\dot{\theta}_{1} \\
\dot{\theta}_{2}
\end{array}\right] \\
& \left\{\begin{array}{c}
\ddot{x}_{p} \\
\ddot{y}_{p} *
\end{array}\right\}=[\mathrm{J}]\left\{\begin{array}{l}
\ddot{\theta}_{1} \\
\ddot{\theta}_{2}
\end{array}\right\}
\end{aligned}
$$


where $J$ is the Jacobian matrix. For the two axis robot under consideration

$$
[J]=\left[\begin{array}{cc}
-\left\{a_{1} \sin \theta_{1}+a_{2} \sin \left(\theta_{1}+\theta_{2}\right)\right\} & -\left\{a_{2} \sin \left(\theta_{1}+\theta_{2}\right)\right\} \\
\left\{a_{1} \cos \theta_{1}+a_{2} \cos \left(\theta_{1}+\theta_{2}\right)\right\} & \left\{a_{2} \cos \left(\theta_{1}+\theta_{2}\right)\right\}
\end{array}\right]
$$

Thus, the kinematic parameters of the robot can be determined as follows:

$$
\left\{\begin{array}{l}
\Delta \theta_{1} \\
\Delta \theta_{2}
\end{array}\right\}=[\mathrm{J}]^{-1}\left\{\begin{array}{c}
\Delta x_{p} \\
\Delta y_{p}
\end{array}\right]
$$

and

$$
\begin{aligned}
& \left(\theta_{i}\right)_{\text {new }}=\left(\theta_{i}\right)_{\text {old }}+\Delta \theta_{i} \\
& \left\{\begin{array}{l}
\dot{\theta}_{1} \\
\dot{\theta}_{2}
\end{array}\right\}=[\mathrm{J}]^{-1}\left\{\begin{array}{c}
\dot{x}_{p} \\
\dot{y}_{p}
\end{array}\right\} \\
& \left\{\begin{array}{c}
\ddot{\theta}_{1} \\
\ddot{\theta}_{2}
\end{array}\right\}=[\mathrm{J}]^{-1}\left\{\begin{array}{c}
\ddot{x}_{p}^{*} \\
\ddot{y}_{p}^{*}
\end{array}\right\}
\end{aligned}
$$

where

$$
\begin{aligned}
\ddot{x}_{p}^{*}= & \ddot{x}_{p}+\left\{a_{1} \cos \theta_{1}+a_{2} \cos \left(\theta_{1}+\theta_{2}\right)\right\} \dot{\theta}_{1}^{2} \\
& +\left\{2 a_{2} \cos \left(\theta_{1}+\theta_{2}\right)\right\} \dot{\theta}_{1} \dot{\theta}_{2} \\
& +\left\{a_{2} \cos \left(\theta_{1}+\theta_{2}\right)\right\} \dot{\theta}_{2}^{2} \\
\ddot{y}_{p}^{*}= & \ddot{y}_{p}+\left\{a_{1} \sin \theta_{1}+a_{2} \sin \left(\theta_{1}+\theta_{2}\right)\right\} \dot{\theta}_{1}^{2} \\
& +\left\{2 a_{2} \sin \left(\theta_{1}+\theta_{2}\right)\right\} \dot{\theta}_{1} \dot{\theta}_{2} \\
& +\left\{a_{2} \sin \left(\theta_{1}+\theta_{2}\right)\right\} \dot{\theta}_{2}^{2}
\end{aligned}
$$


Having determined the joint variables, the torque expressions in Equations ( 8 ) and (9), and the objective function in Equation (1) can be evaluated.

A comparison was made between the optimal and standard solutions at $T=0.45$ seconds. Figure 30 shows the resulting motion at joint one. The optimal motion has an initial dwell in its motion. The standard solution has an overshoot in its initial motion in order that the straight line end effector path can be tracked. Figure 31 shows that the motions at joint two are nearly identical. Figures 32 and 33 compare the resulting torque profiles at joints one and two, respectively. Figure 32 shows a definite improvement of the optimal motion over the standard solution. The standard solution can be. seen to vary between -18 and 100 Newtonmeters. The optimal motion program produces a smooth torque profile which varies between 30 and 50 Newton-meters. Figure 33 shows that the nature of the torque profiles at joint two are similar, but the optimal solution produces lower peak values of torque. Figure 34 compares the resulting end effector paths. The standard solution is a straight line as prescribed. However, the optimal solution still provides a regular, direct path from $A$ to $B$. Thus, it can be seen that the optimal solution is superior to the standard solution.

The value of the objective function for the optimal motion is $1=$ 1220. For the standard solution, $I=1730.49$ at $T=0.45$ seconds. Thus, the value of the objective function indicates that the optimal motion should be superior to that of the standard solution as shown. However, this does not guarantee that the optimal time for the standard solution is $T=0.45$ seconds. In order to determine the optimal time, an interactive program was written so that different traveling times could be 


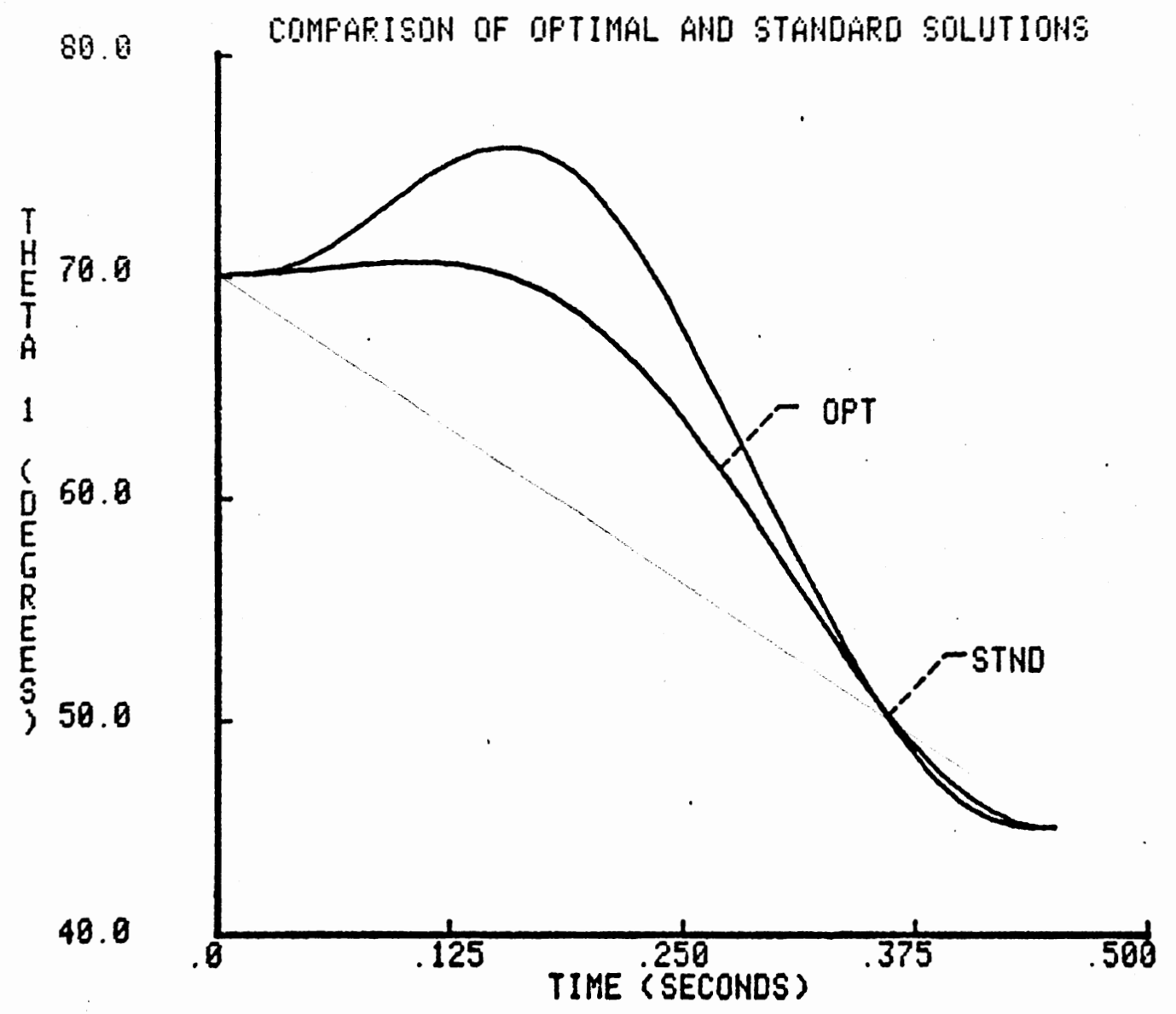

Figure 30. Comparison of Joint 1 Displacements for Optimal and Standard Solutions. 


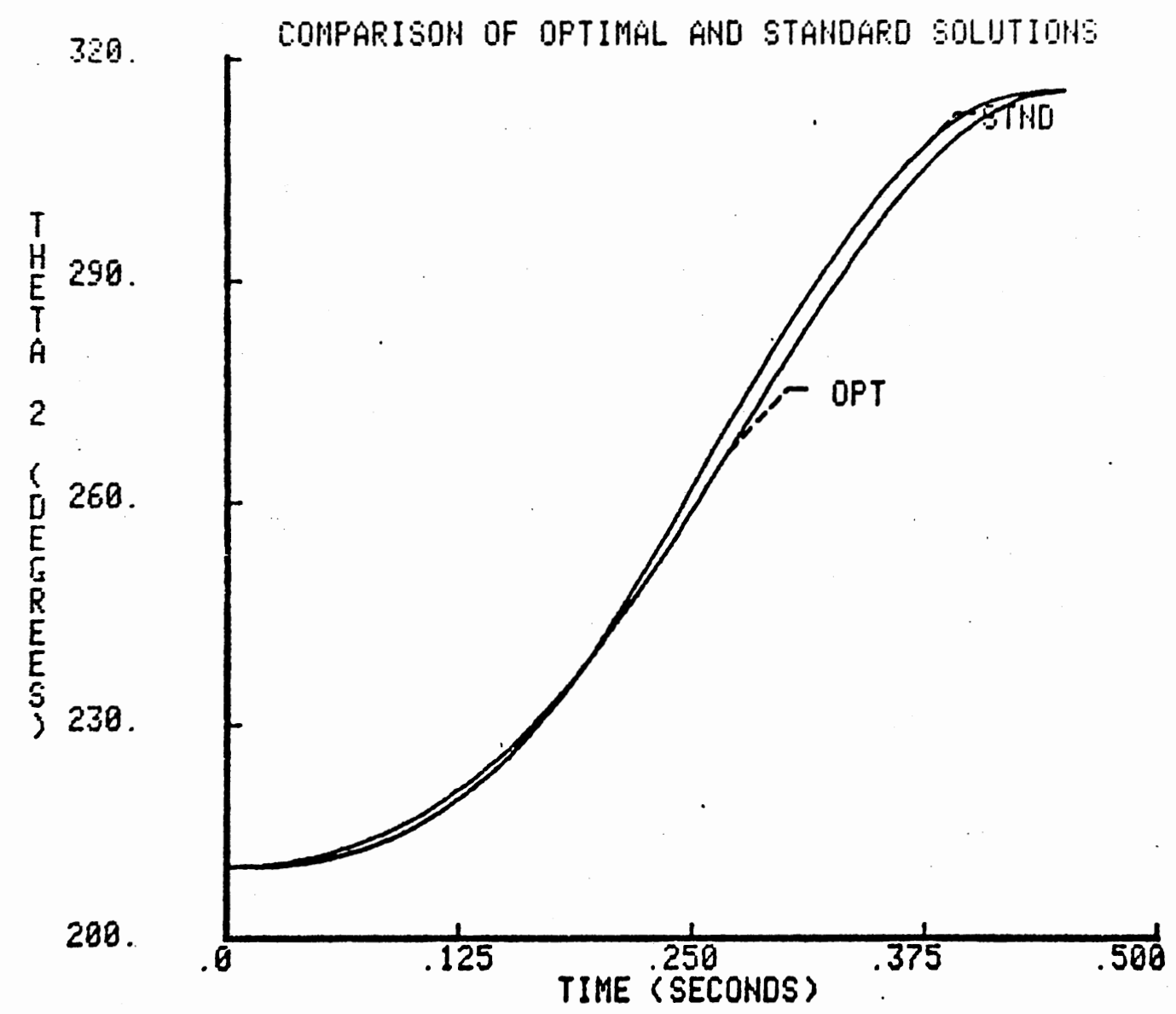

Figure 31. Comparison of Joint 2 Displacements for Optimal and Standard Solutions. 


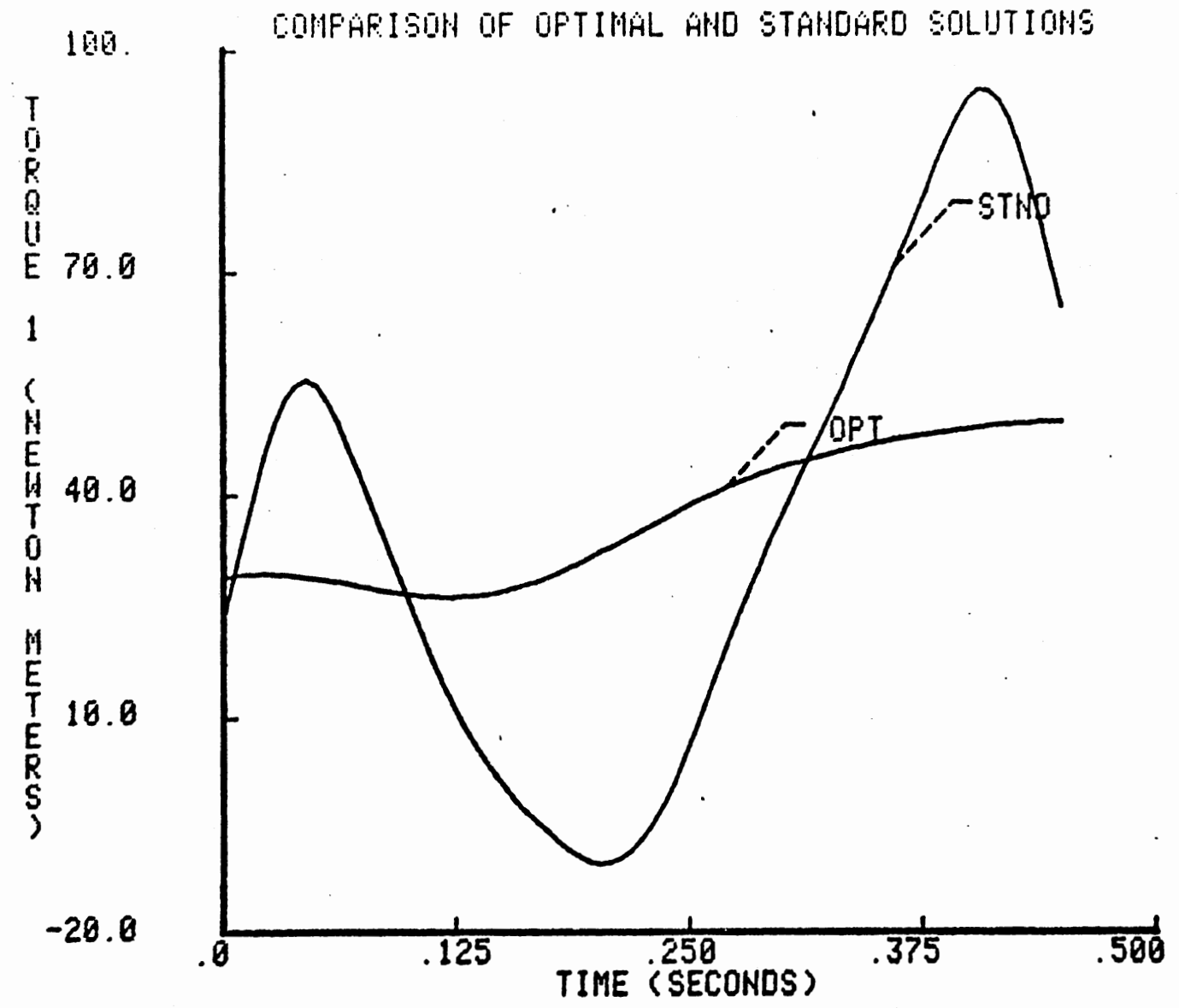

Figure 32. Comparison of Joint 1 Torques for Optimal and Standard Solutions. 


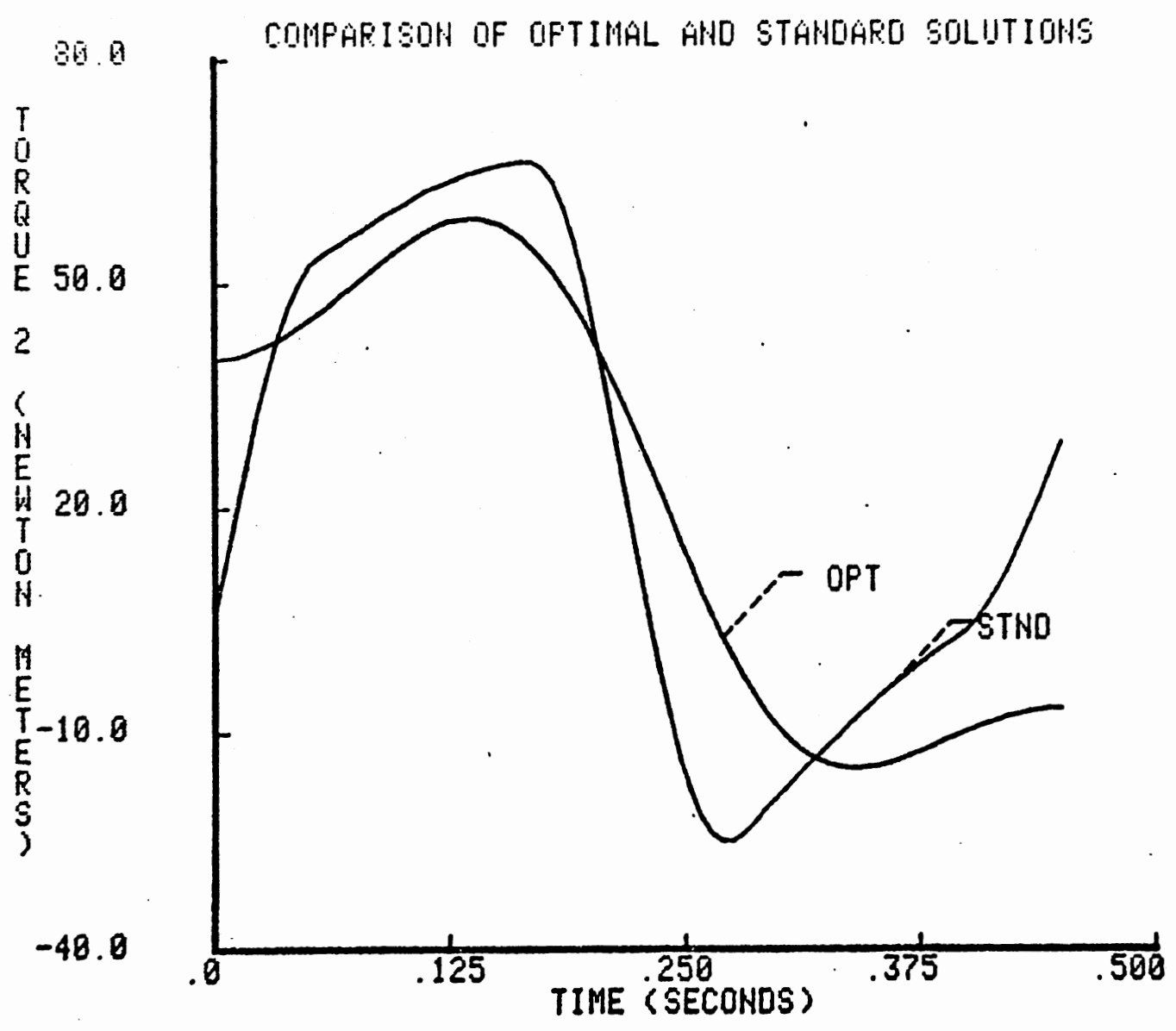

Figure 33. Comparison of Joint 2 Torques for Optimal and Standard Solutions. 


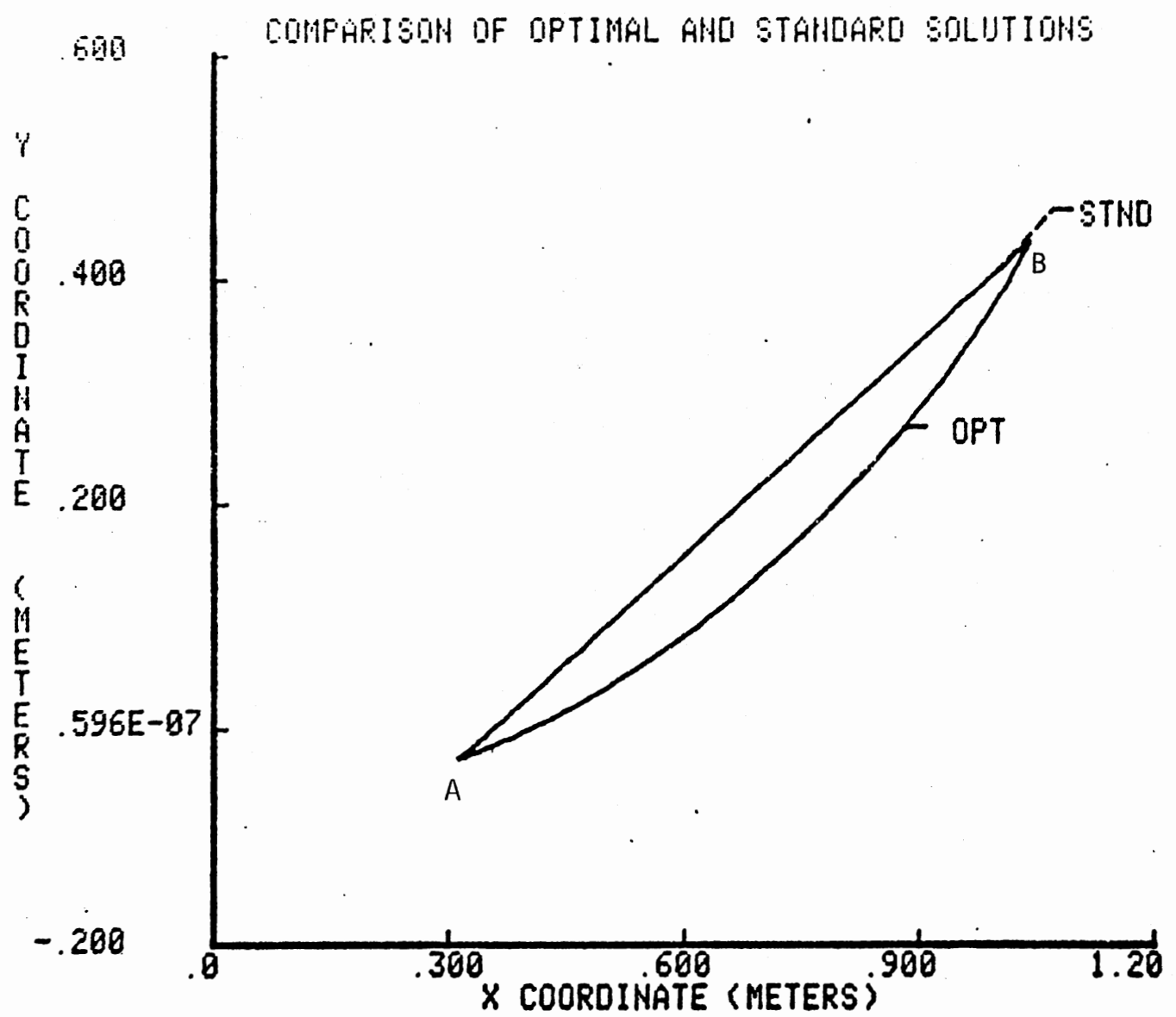

Figure 34. Comparison of End Effector Paths for Optimal and Standard Solutions.

ซึ้ 
evaluated. The results are shown in Table $V$. In examining the table, it can be seen that the optimal traveling time for the standard solution is $T=0.55$ seconds. The objective function value is $1=1623.56$. This is not an improvement over the optimal solution of $1=1220$ at $T=0.45$ seconds.

\subsection{Optimal Motion Programming for New Workstations}

In this section, an optimal motion program for the two axis robot will be obtained for moving an object between two new workstations. The kinematic and dynamic properties of the manipulator.will remain the same. Only the initial and final configurations of the manipulator will change. Figure 35 shows the relationship between the old and new workstations. $A$ and $B$ represent the old location; $A^{\prime}$ and $B^{\prime}$ represent the new location. The boundary conditions for the robot at the new workstations are as follows:

$$
\begin{array}{ll}
\theta_{1}(0)=70.0^{\circ} & \theta_{2}(0)=245.0^{\circ} \\
\theta_{1}(T)=70.0^{\circ} & \theta_{2}(T)=335.0^{\circ} \\
\dot{\theta}_{1}(0)=0.0 & \dot{\theta}_{2}(0)=0.0 \\
\dot{\theta}_{1}(T)=0.0 & \dot{\theta}_{2}(T)=0.0
\end{array}
$$

The purpose of determining an optimal motion program for a new task has a dual purpose. First, it allows the results of the previous sections to be applied to a new problem to determine if they hold in general. Second, it will be possible to make some comparison between the optimal motion programs obtained by varying the position of the workstations with respect to the robot. This is possible because the new workstations, 
TABLE V

DETERMINATION OF OPTIMAL TIME FOR STANDARD SOLUTION

\begin{tabular}{cc}
\hline $\begin{array}{c}\text { Cycle Time, } \\
\text { (Seconds) }\end{array}$ & Objective Function, I \\
\hline 0.25 & 5781.06 \\
0.45 & 1730.49 \\
0.50 & 1636.29 \\
0.55 & 1623.56 \\
0.60 & 1659.49 \\
0.65 & 1725.64 \\
1.0 & 2486.19 \\
\hline
\end{tabular}




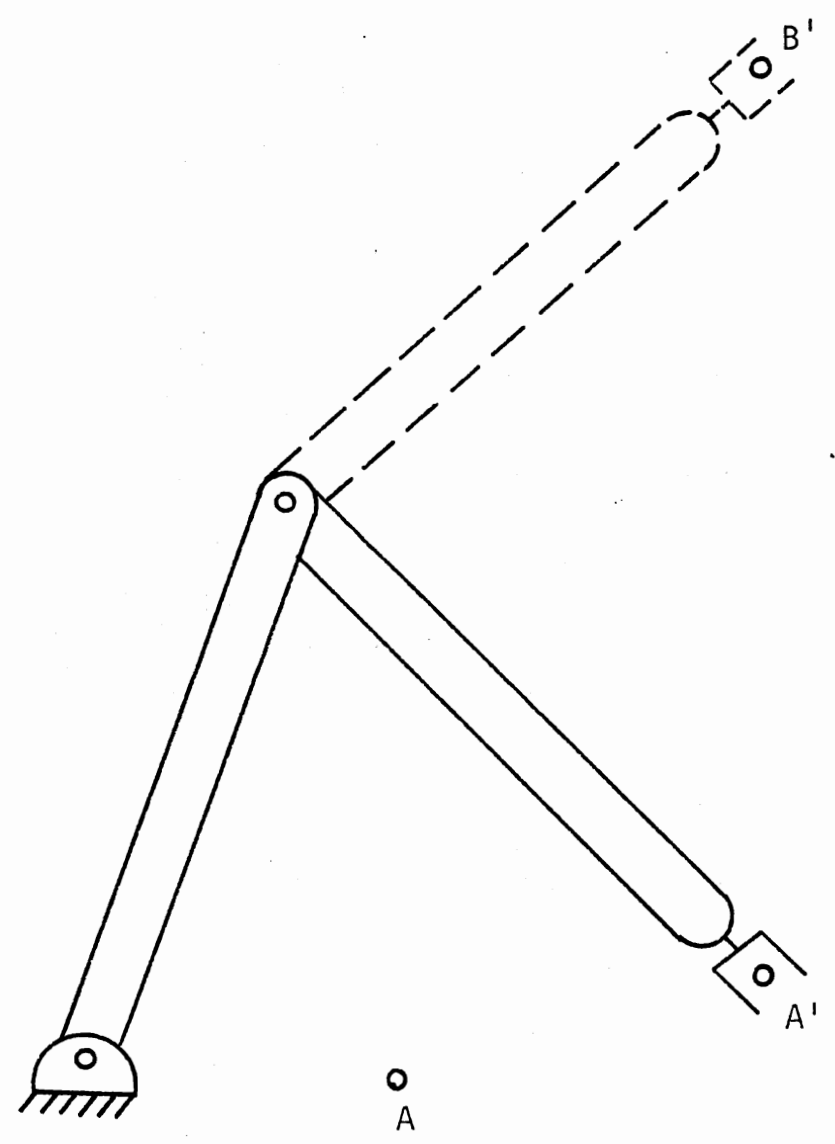

0

Figure 35. Second Task Description 
$A^{\prime}$ and $B^{\prime}$, have the same distance between them as $A$ and $B$. Thus, the workstations can be interpreted as representing the same task.

The first factor to be determined is the optimal traveling time from $A^{\prime}$ to $B^{\prime}$. The comparisons made in section 3.6 indicate that objective function 1 (integral over the traveling time of the sum of the square of the joint torques) is best suited for determining the optimal traveling time. This objective function was applied using a one term trigonometric approximating function. The results are shown in Table VI. An optimal traveling time of 0.69 seconds is indicated with the objective function value $1=1605.0$

Having determined the optimal traveling time, increasing term trigonometric approximations were carried out to determine how many terms are required to accurately approximate the optimal joint motions. The comparisons in section 3.6 indicate that objective functions 1 and 3 provide acceptable results at the optimal traveling time. Both were applied with the increasing term approximations. The results are shown in Table VII. In examining Table VII, it can be seen that the objective function value continually improves from the zero term approximation through the three term approximation. This is true for both objective functions. However, it can be seen that the improvement in going from the two term to three term solution is small compared to the other changes. Thus, adding two terms to the $\phi_{0}(t)$ solution will accurately approximate the optimal motions. By comparing the weighting coefficients of the converged point for the two objective functions, it can be seen that the solutions will provide different motions at joint one, but nearly identical motions at joint two. Figures 36 and 37 compare the resulting joint torques for the objective functions using the two term approximations. It can be seen 
TABLE VI

DETERMINATION OF OPTIMAL TRAVELING TIME

FOR NEW WORKSTATIONS

\begin{tabular}{|c|c|c|}
\hline Starting Point & Converged Point & Objective \\
\hline$c_{11}=0.0 \quad c_{21}=0.0$ & $c_{11}=0.1766 \quad c_{21}=-0.2615$ & 1606.0 \\
\hline$T=0.25$ & $T=0.6878$ & \\
\hline$c_{11}=0.0 \quad c_{21}=0.0$ & $c_{11}=0.1721 \quad c_{21}=-0.2594$ & 1605.0 \\
\hline$T=0.50$ & $T=0.6883$ & \\
\hline$c_{11}=0.0 \quad c_{21}=0.0$ & $c_{11}=0.1760 \quad c_{21}=-0.2684$ & 1605.0 \\
\hline$T=1.0$ & $T=0.6985$ & \\
\hline$c_{11}=0.0 \quad c_{21}=0.0$ & $c_{11}=0.3106 \quad c_{21}=-0.7723$ & 1958.1 \\
\hline$T=1.5$ & $T=1.34$ & \\
\hline
\end{tabular}


TABLE VII

COMPARISON OF INCREASING TERM TRIGONOMETRIC APPROXIMATIONS AT $T=0.69$ SECONDS

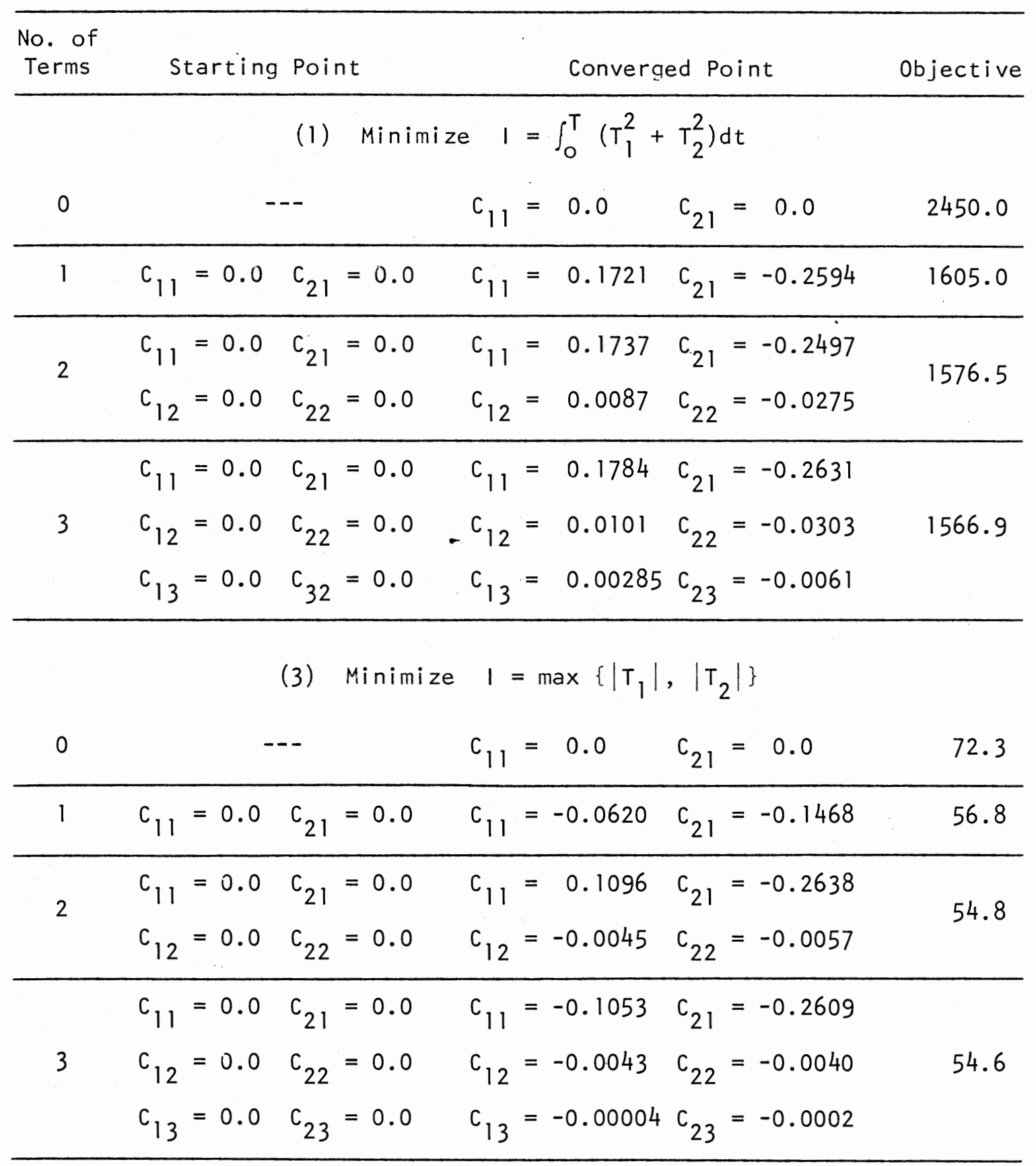




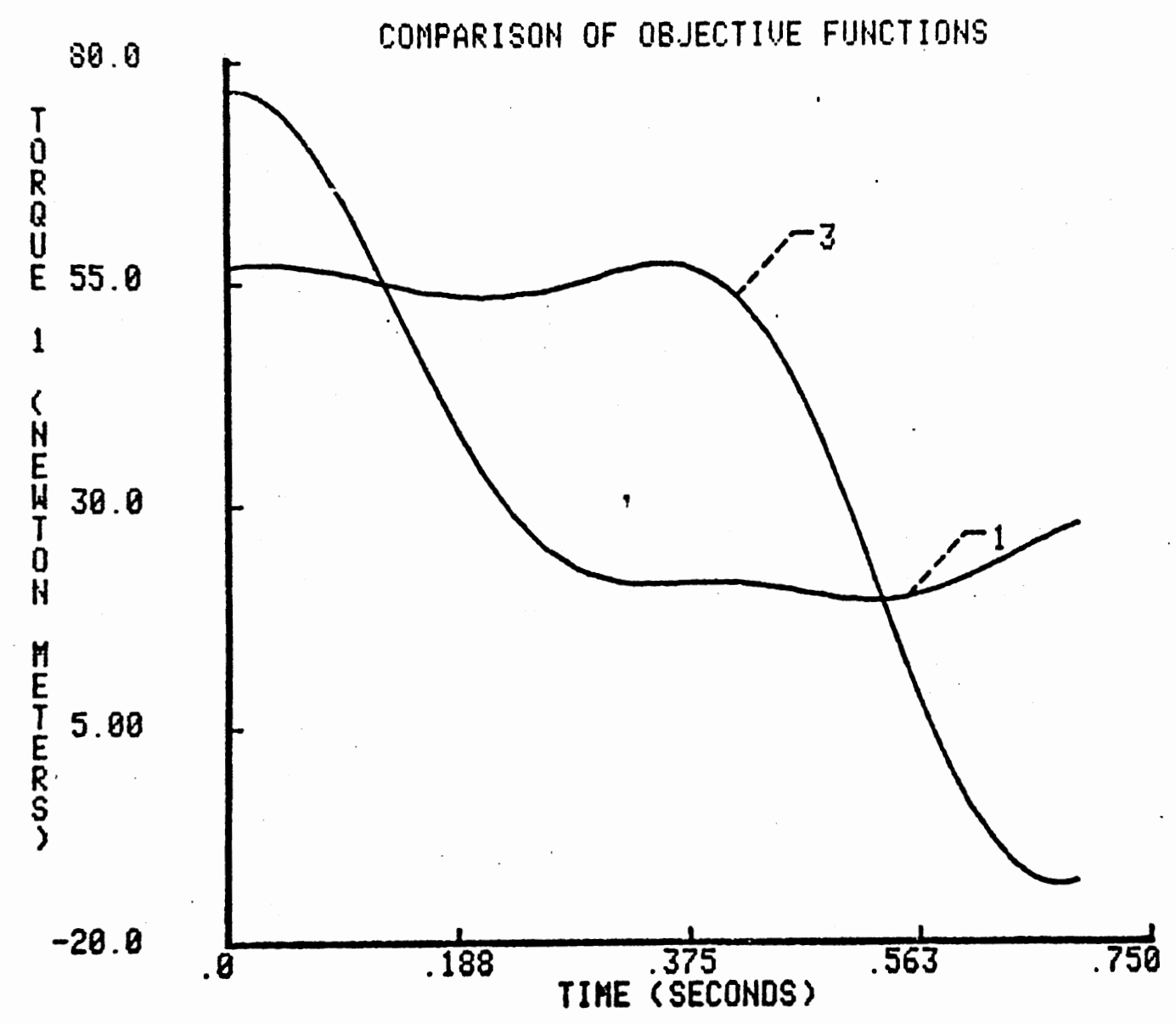

Figure 36. Joint 1 Torques for Objective Functions 1 and 3 . 


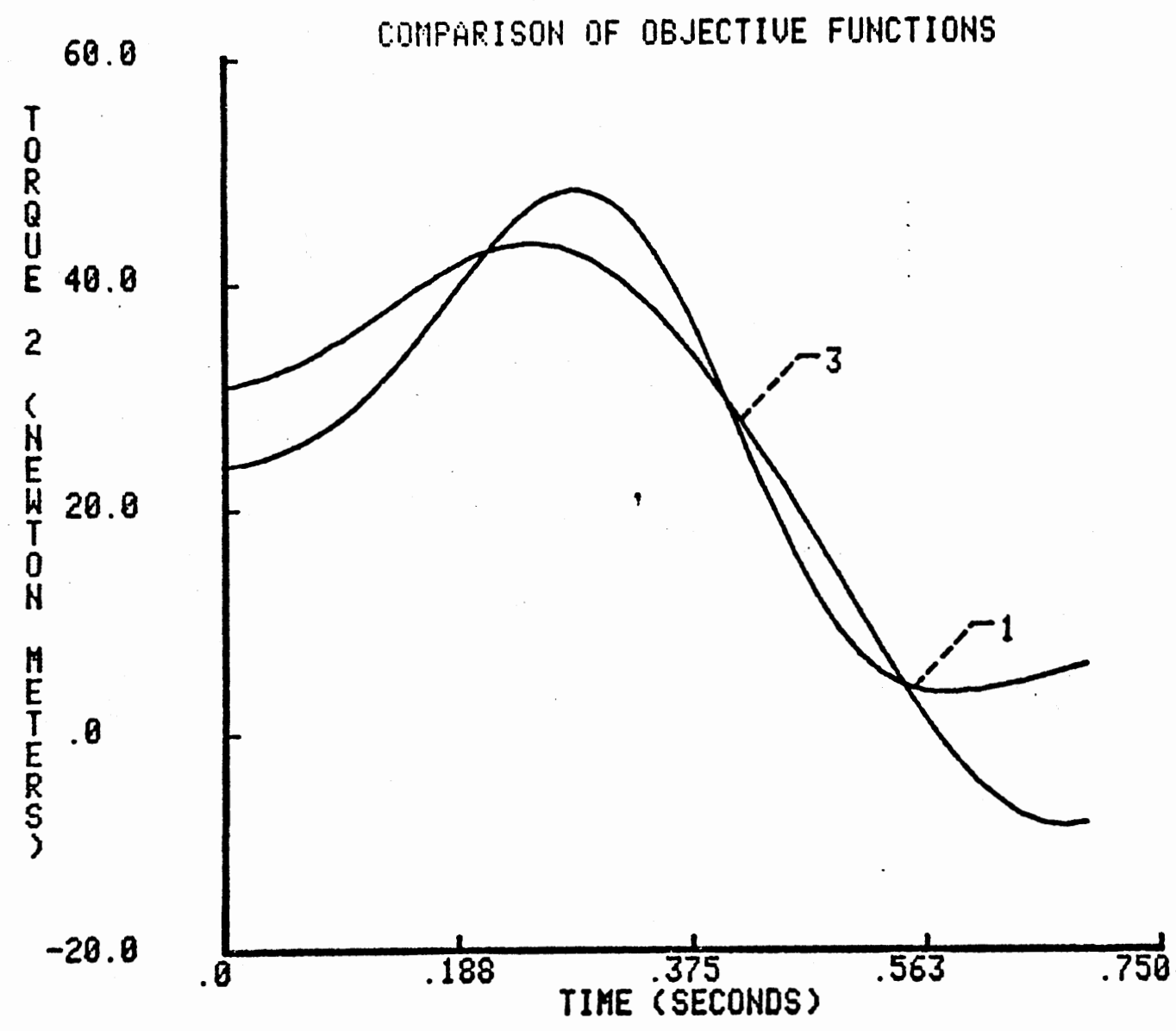

Figure 37. Joint 2 Torques for Objective Functions 1 and 3. 
that objective function 3 provides a lower value of peak torque at both joints.

Choosing the approximation provided by objective function 3 as the optimal motion program with respect to joint torques, a comparison was made between the optimal and the standard straight line motion programs. Figures 38 through 42 compare the results for these motions. Figure 38 shows the displacement at joint one as a function of time. The standard solution has a large overshoot in order that it can track the straight line end effector path. The optimal solution reduces the overshoot considerably. Figure 39 shows that the displacement at joint two is nearly identical for the optimal and standard solutions.

Figure 40 compares the torque versus time characteristics at joint one. The optimal solution reduces the peak torque from 75 to 55 Newtonmeters. Also, the optimal solution provides a smoother torque profile over the entire traveling time. Figure 41 compares the torques at joint two. The characteristics of the optimal and standard profiles are nearly the same. The optimal solution slightly reduces the magnitude of the peak torque. Figure 42 compares the resulting end effector paths. The standard solution provides a straight line path between $A^{\prime}$ and $B^{\prime}$ as prescribed. The optimal solution takes a curved path. The optimal end effector path provides a regular and direct path between $A^{\prime}$ and $B^{\prime}$, and is acceptable for actual use.

A comparison can now be made on how the location of the workstations with respect to the manipulator affects the optimal motion program. As mentioned previously, the task described in this section is the same as that used in previous sections in that the distance between points $A^{\prime}$ and $B^{\prime}$ is the same. Figures 43 and 44 compare the joint torques for the two 


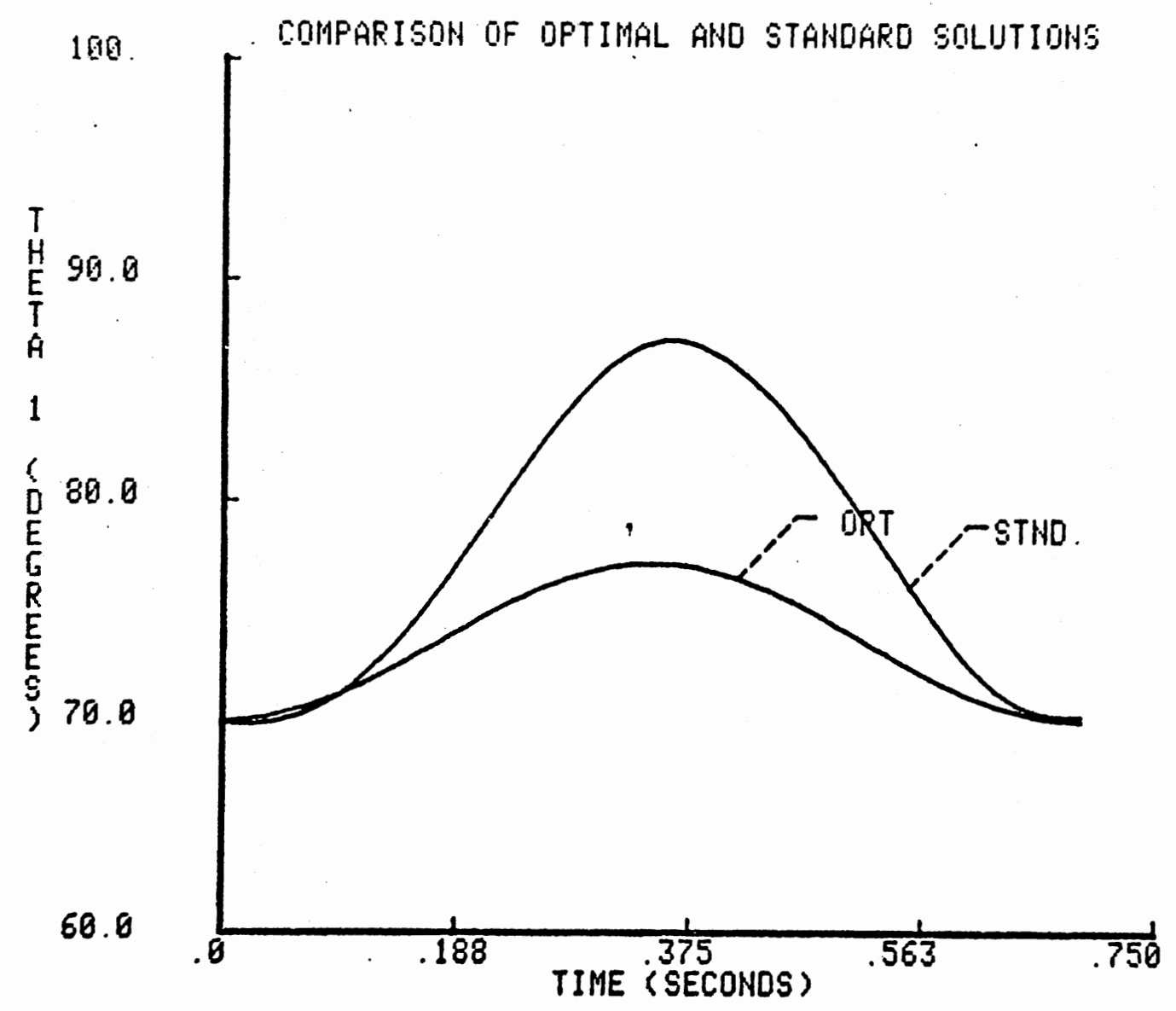

Figure 38. Comparison of Displacements at Joint 1 for Optimal and Standard Solutions. 


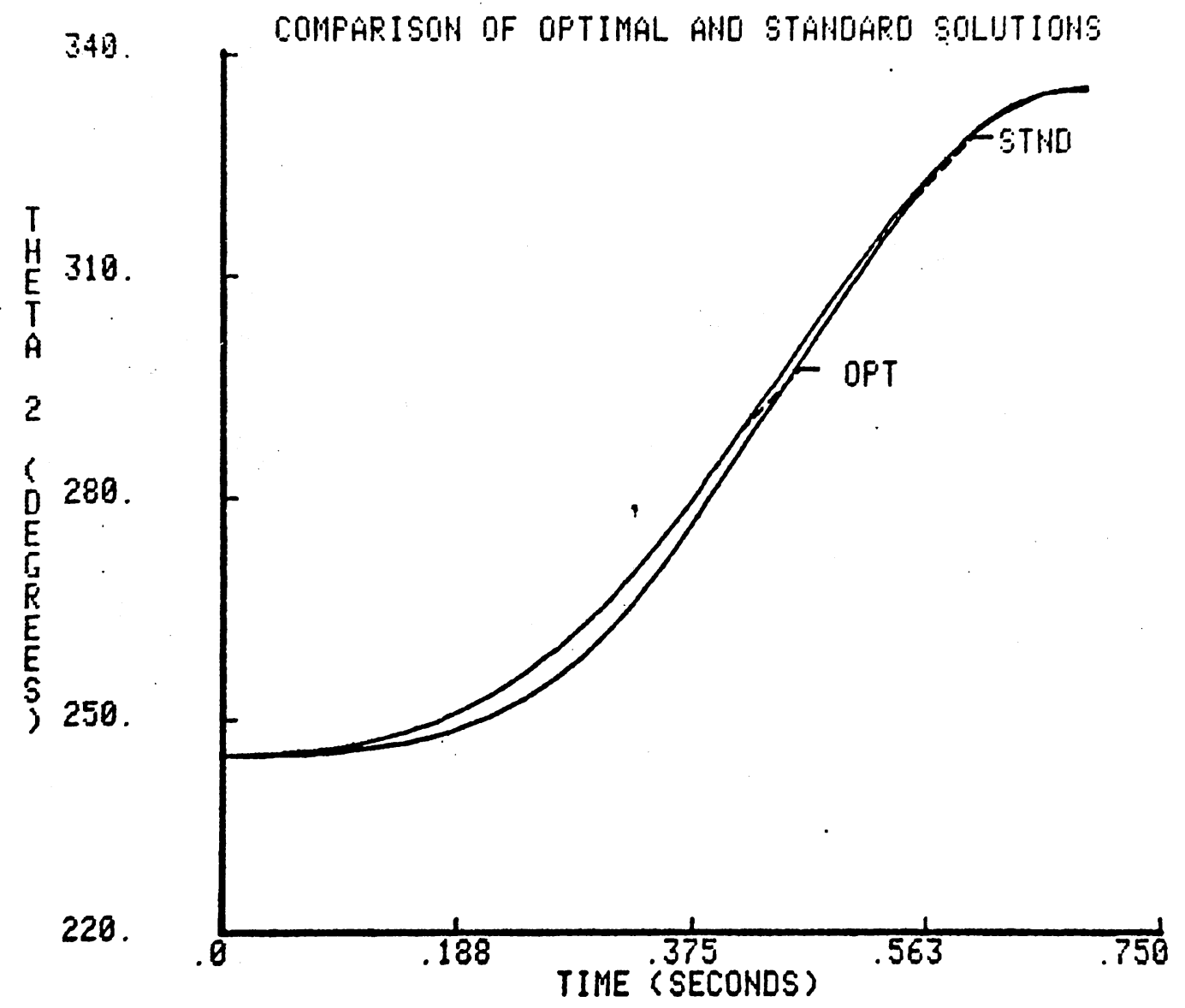

Figure 39. Comparison of Displacements at Joint 2 for Optimal and Standard Solutions. 


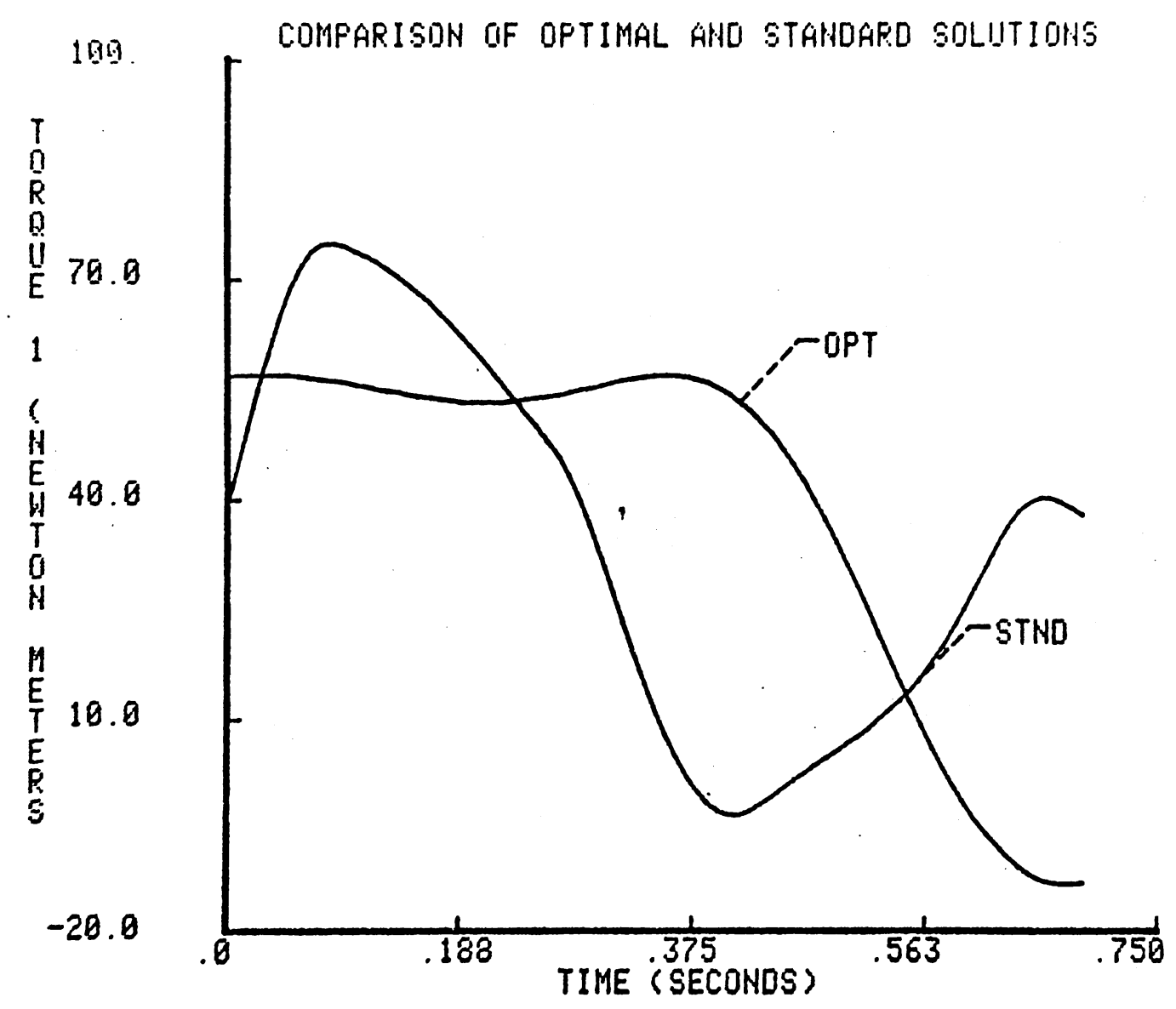

Figure 40. Comparison of Torques at Joint 1 for Optimal and Standard Solutions. 


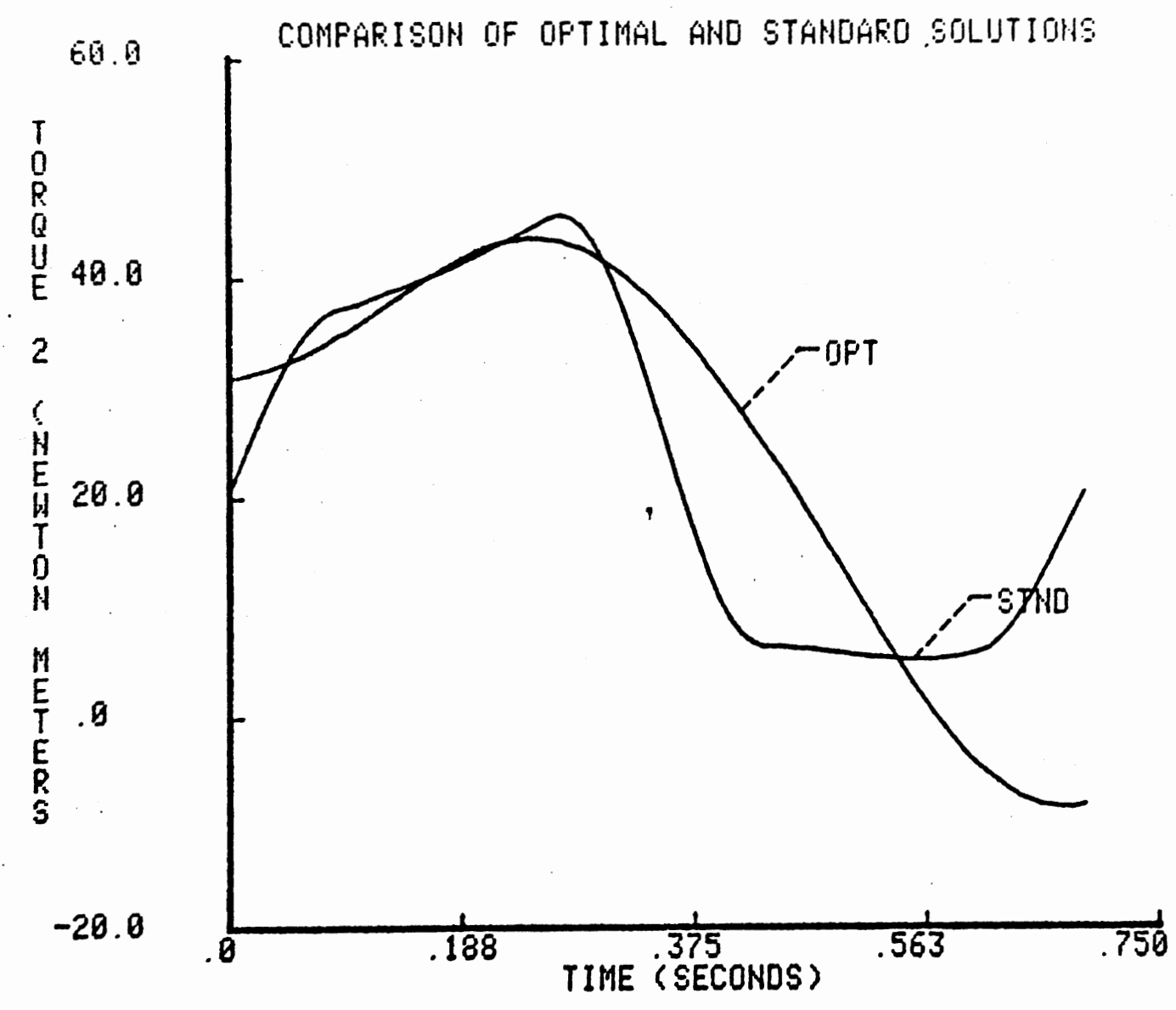

Figure 41. Comparison of Torques at Joint 2 for Optimal and Standard Solutions. 


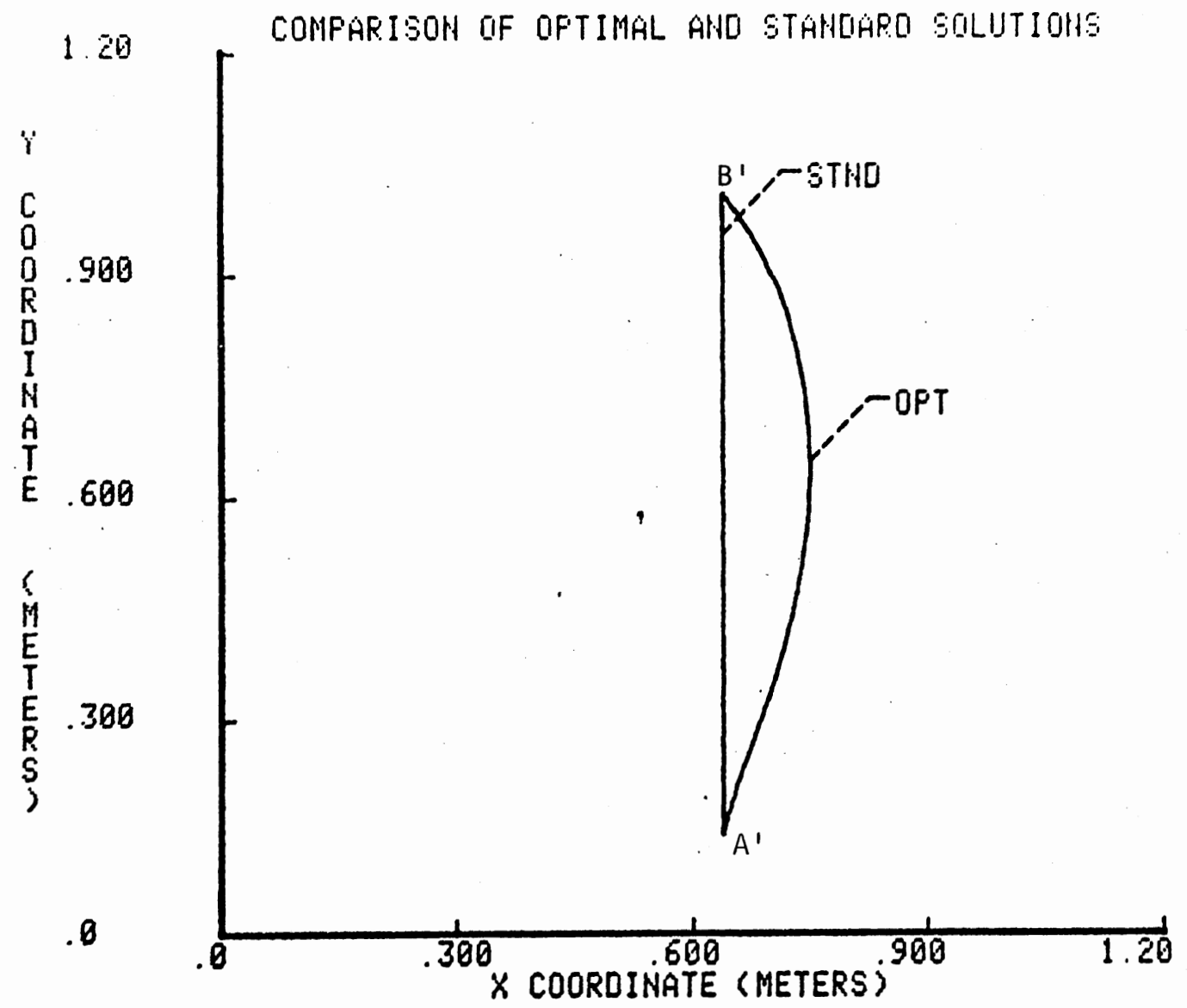

Figure 42. Comparison of End Effector Paths for Optimal and Standard Solutions. 


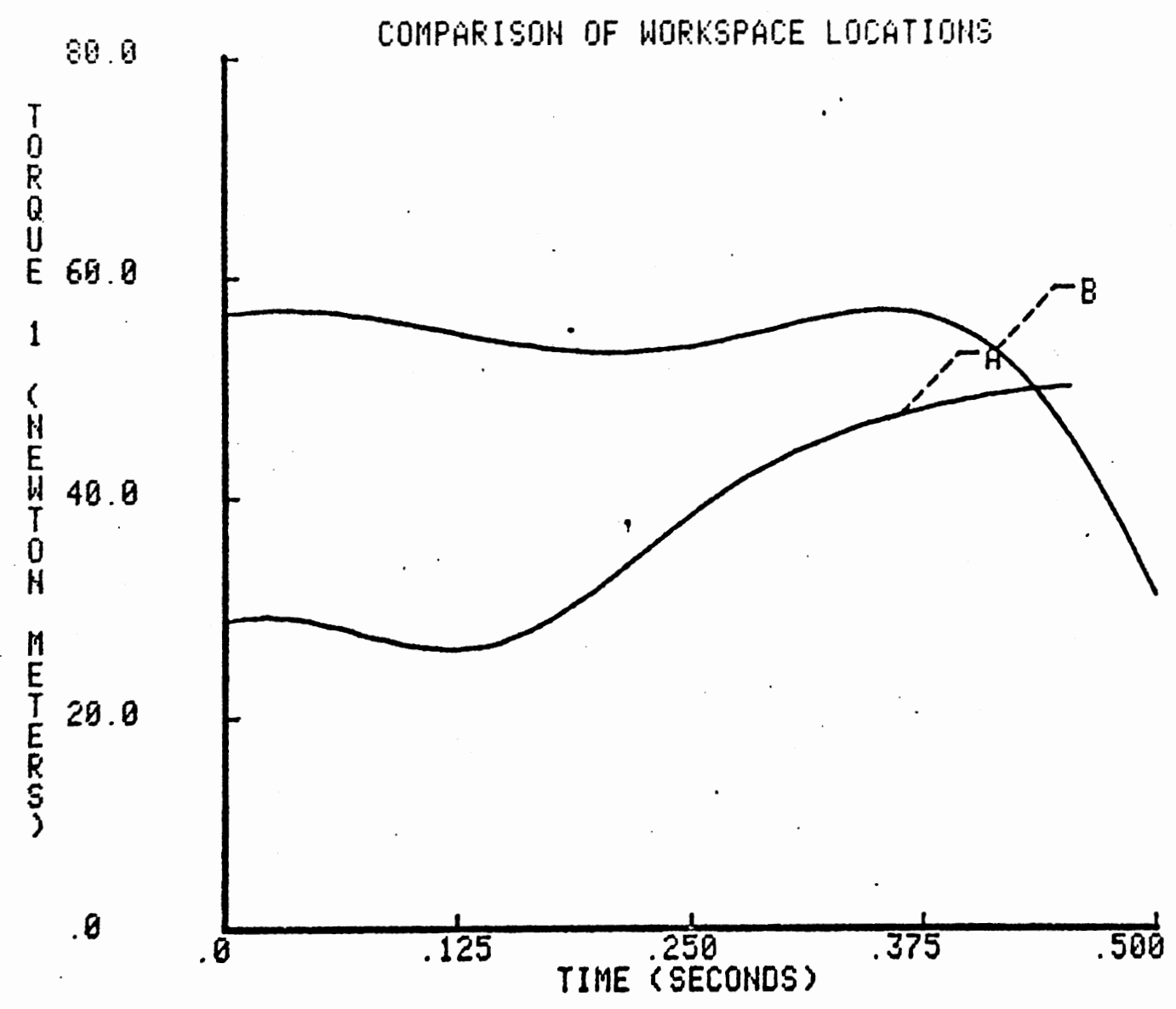

Figure 43. Torques at Joint 1 for Different Workspace Locations. 


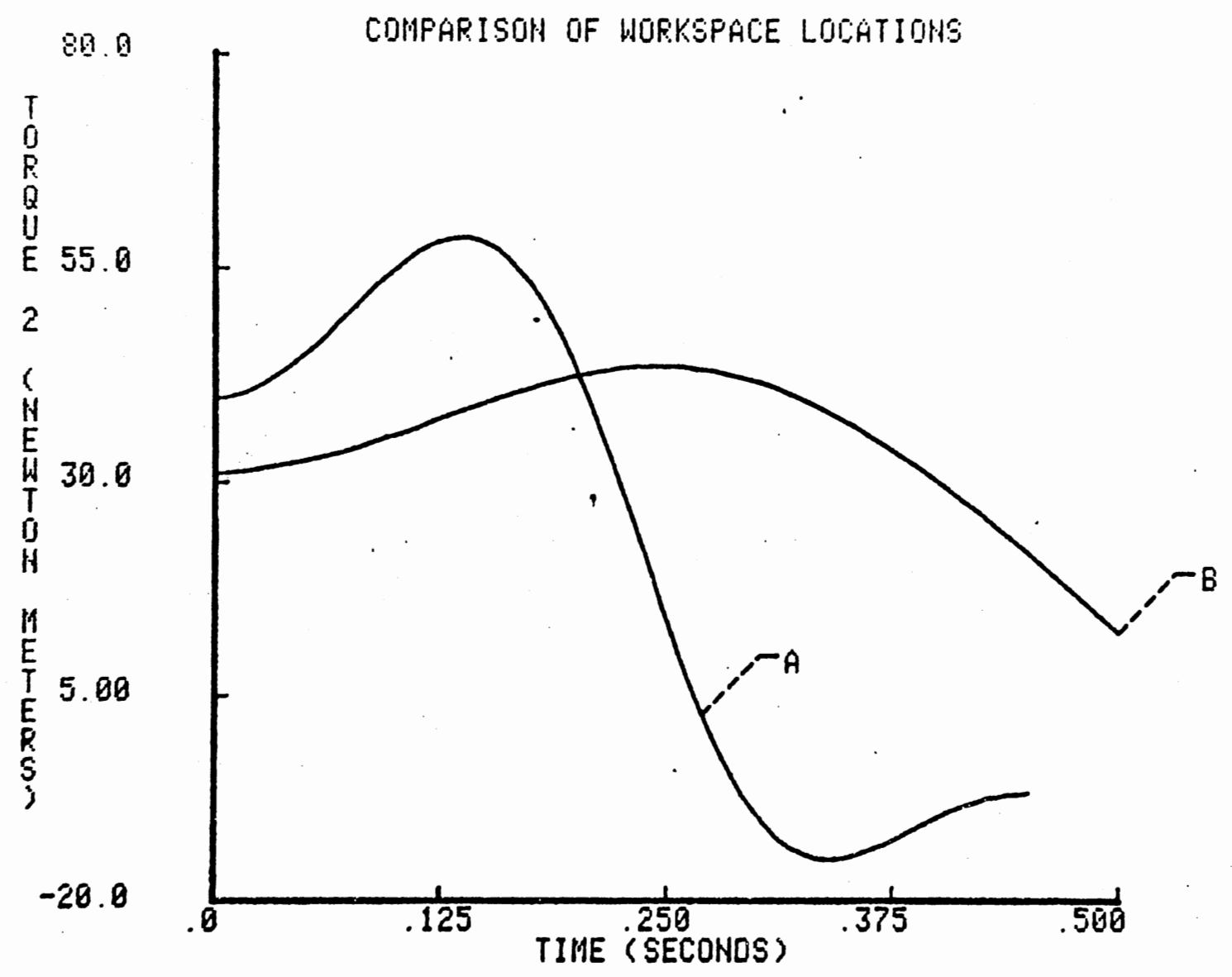

Figure 44. Torques at Joint 2 for Different Workspace Locations . 
task locations within the workspace of the robot (see Figure 35). Curve A represents the optimal motion program in moving between $A$ and $B$. Curve $B$ represents the optimal motion for workstations $A^{\prime}$ and $B^{\prime}$. Figure 43 shows that task location A provides a superior torque profile at joint one. Not only is the peak magnitude of the torque reduced, but no rapid changes in torque occur as at the end of optimal program B. The opposite conclusion is drawn at joint two as shown in Figure 44. Optimal motion program B provides a smaller peak torque and a smoother torque profile than that of program A.

It is difficult to conclude which motion program is superior due to the division of superiority at each joint. However, either task location. would be acceptable. The important fact to be realized is that not only is it important to determine an optimal motion program for a prescribed task, but also to determine the optimal location of the task with respect to the manipulator. 
CHAPTER IV

\section{APPLICATION OF THE THEORY TO A 3-R ROBOT}

\subsection{Scope of the Chapter}

In this chapter, the theory developed in Chapter 11 will be applied to a three link, three revolute (3R) robot manipulator. The configuration of the $3 R$ robot is shown in Figure 45 . Notice that the configuration of the robot is such that it will move in three dimensions. The optimal motion program obtained will be compared to the standard straight line motion as presented in the previous chapter.

\subsection{Problem to be Solved}

The task to be performed by the manipulator is a simple lifting task from a station $C$ to a station $D$ as indicated in Figure 45 . Note the $x, y, z$ coordinates at each workstation. The workstations are such that changes in all three coordinate directions occur, and the manipulator will be required to have three dimensional motion. The manipulator joints will be required to start at station $\mathrm{C}$ with zero angular velocity and end at station $D$ with zero angular velocity. The boundary conditions are specified as follows:

$$
\begin{array}{lll}
\theta_{1}(0)=0.0^{\circ} & \theta_{2}(0)=70.0^{\circ} & \theta_{3}(0)=210.0^{\circ} \\
\theta_{1}(T)=60.0^{\circ} & \theta_{2}(T)=45.0^{\circ} & \theta_{3}(T)=315.0^{\circ} \\
\dot{\theta}_{1}(0)=0.0 & \dot{\theta}_{2}(0)=0.0 & \dot{\theta}_{3}(0)=0.0 \\
\dot{\theta}_{1}(T)=0.0 & \dot{\theta}_{2}(T)=0.0 & \dot{\theta}_{3}(T)=0.0
\end{array}
$$




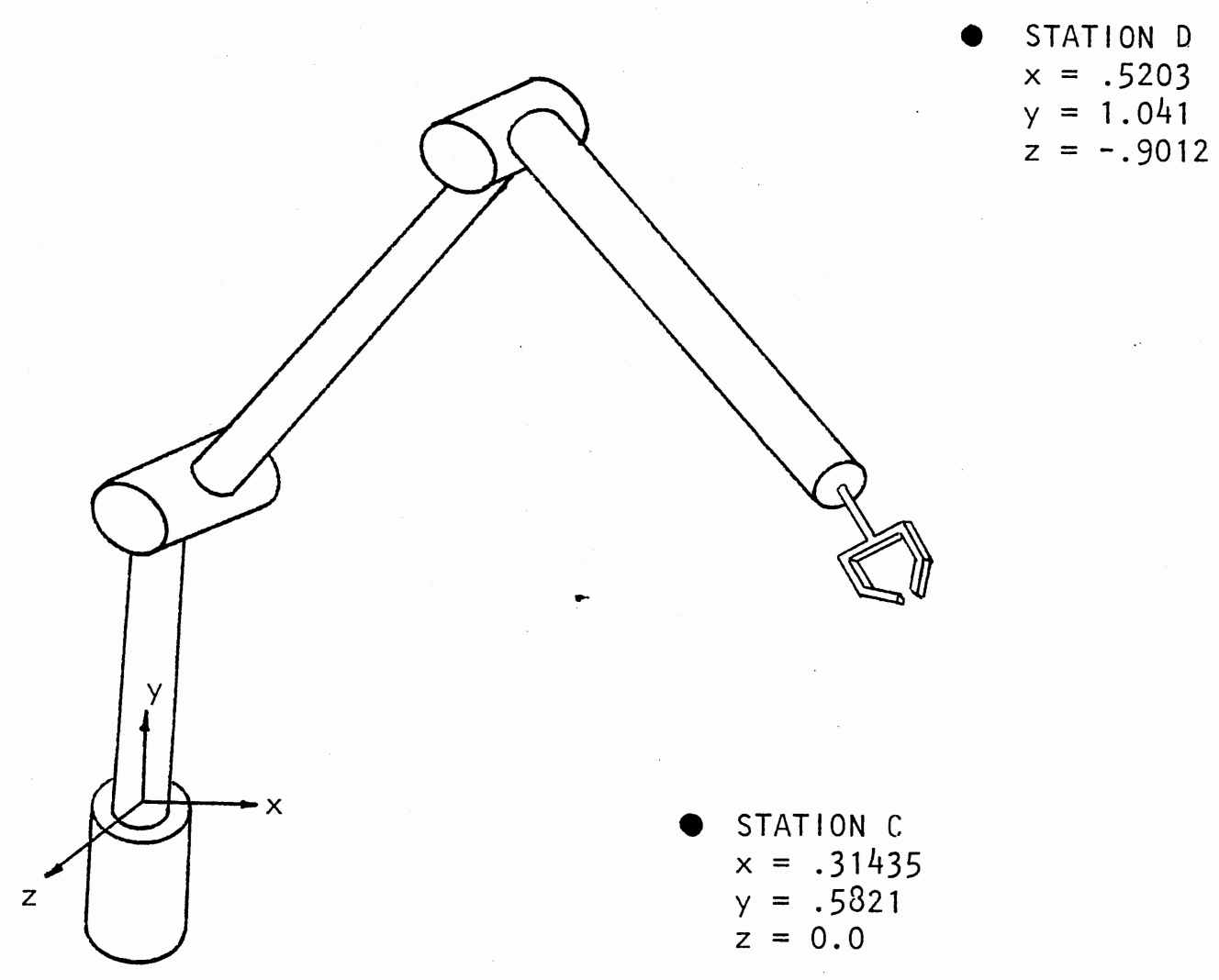

Figure 45. Three-Axis Robot 
Figure 46 shows a free body diagram for the three axis robot. The variables are shown on the diagram, and the values chosen for the example problem are given below.

$$
\begin{aligned}
& a_{1}=0.6096 \mathrm{~m} \quad a_{2}=0.6096 \mathrm{~m} \quad a_{3}=0.6096 \mathrm{~m} \\
& l_{1}=0.3048 \mathrm{~m} \quad l_{2}=0.3048 \mathrm{~m} \quad l_{3}=0.4432 \mathrm{~m} \\
& m_{1}=12.02 \mathrm{~kg} \quad \mathrm{~m}_{2}=12.02 \mathrm{~kg} \quad \mathrm{~m}_{3}=22.02 \mathrm{~kg} \\
& \mathrm{I}_{33}=0.0126 \mathrm{~kg} \cdot \mathrm{m}^{2} \quad \mathrm{I}_{33}=0.3785 \mathrm{~kg} \cdot \mathrm{m}^{2} \quad \mathrm{I}_{33}=0.8857 \mathrm{~kg} \cdot \mathrm{m}^{2} \\
& \mathrm{I}_{22}=0.3785 \mathrm{~kg} \cdot \mathrm{m}^{2} \quad \mathrm{I}_{22}=0.8857 \mathrm{~kg} \cdot \mathrm{m}^{2} \\
& 1_{11}=0.0126 \mathrm{~kg} \cdot \mathrm{m}^{2} \quad 1_{11}=0.0231 \mathrm{~kg} \cdot \mathrm{m}^{2}
\end{aligned}
$$

where

$$
\begin{aligned}
& 1_{33}=\text { moment of inertia about } z \text { axis; } \\
& 1_{22}=\text { moment of inertia about } y \text { axis; and } \\
& 1_{11}=\text { moment of inertia about } x \text { axis. }
\end{aligned}
$$

Note: The inertial properties of the load carried at the end effector are included in the third link.

The expressions for the torque at each joint of the robot will be required so that objective functions 1 and 3 of the previous chapter can be evaluated. In order to obtain the torque expressions, the kinematic parameters of the three link robot must be determined. The parameters of interest are the acceleration expressions at the center of gravity of each link and the position, velocity and acceleration expressions for the end point of the third link (point $P$ in Figure 45). The expressions for the point $P$ are given below. They are measured with respect to and expressed in terms of an inertial system located at $0_{1}$.

$$
\begin{aligned}
& x_{p}=a_{2} c_{1} c_{2}+a_{3} c_{1} c_{23} \\
& y_{p}=a_{1}+a_{2} s_{2}+a_{3} s_{23}
\end{aligned}
$$



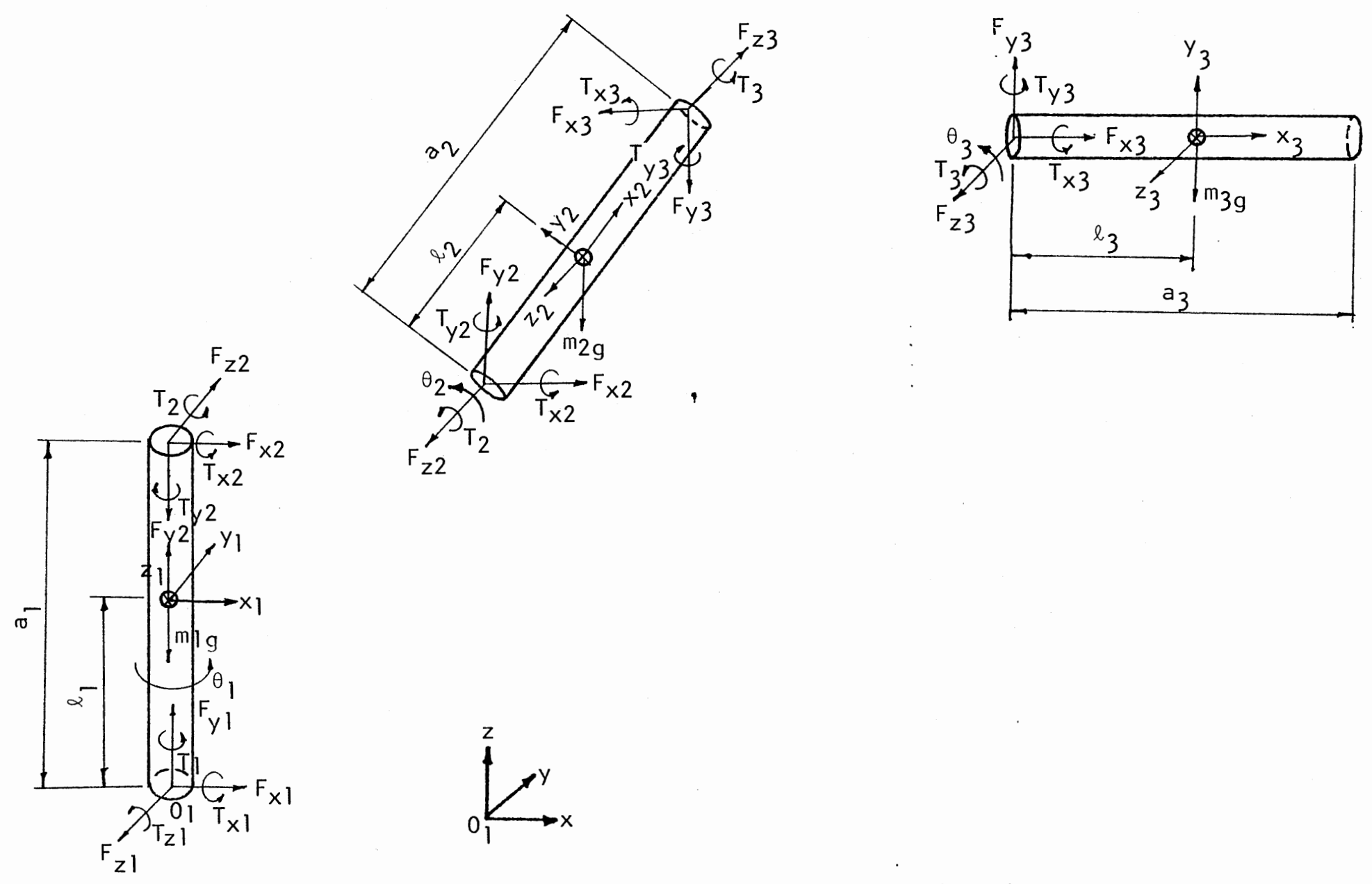

Figure 46. Free-Body Diagram of Three-Axis Robot 


$$
\begin{aligned}
& z_{p}=-a_{2} S_{1} c_{2}-a_{3} s_{1} c_{23} \\
& \dot{x}_{p}=-a_{2} \dot{\theta}_{1} s_{1} c_{2}-a_{2} \dot{\theta}_{2} c_{1} s_{2}-a_{3} \dot{\theta}_{1} s_{1} c_{23}-a_{3}\left(\dot{\theta}_{2}+\dot{\theta}_{3}\right) c_{1} s_{23} \\
& \dot{y}_{p}=a_{2} \dot{\theta}_{2} c_{2}+a_{3}\left(\dot{\theta}_{2}+\dot{\theta}_{3}\right) c_{23} \\
& \dot{z}_{p}=a_{2} \dot{\theta}_{1} c_{1} c_{2}-a_{2} \dot{\theta}_{2} s_{1} s_{2}+a_{3} \dot{\theta}_{1} c_{1} c_{23}-a_{3}\left(\dot{\theta}_{2}+\dot{\theta}_{3}\right) s_{1} s_{23} \\
& \ddot{x}_{p}=\left[-a_{2} s_{1} c_{2}-a_{3} s_{1} c_{23}\right] \ddot{\theta}_{1}+\left[-a_{2} c_{1} c_{2}-a_{3} c_{1} c_{23}\right] \dot{\theta}_{1}^{2} \\
& +\left[2 a_{2} s_{1} s_{2}+2 a_{3} s_{1} s_{23}\right] \dot{\theta}_{1} \dot{\theta}_{2}+\left[2 a_{3} S_{1} S_{23}\right] \dot{\theta}_{1} \dot{\theta}_{3} \\
& +\left[-a_{2} c_{1} s_{2}-a_{3} c_{1} s_{23}\right] \ddot{\theta}_{2}+\left[-a_{2} c_{1} c_{2}-a_{3} c_{1} c_{23}\right] \dot{\theta}_{2}^{2} \\
& +\left[-2 a_{3} c_{1} c_{23}\right] \dot{\theta}_{2} \dot{\theta}_{3}+\left[-a_{3} c_{1} s_{23}\right] \ddot{\theta}_{3}+\left[-a_{3} c_{1} c_{23}\right] \dot{\theta}_{3}^{2} \\
& \ddot{y}_{p}=\left[a_{2} c_{2}+a_{3} c_{23}\right] \ddot{\theta}_{2}+\left[-a_{2} s_{2}-a_{3} s_{23}\right] \dot{\theta}_{2}^{2} \\
& +\left[-2 a_{3} s_{23}\right] \dot{\theta}_{2} \dot{\theta}_{3}+\left[a_{3} c_{23}\right] \ddot{\theta}_{3} \\
& +\left[-a_{3} S_{23}\right] \dot{\theta}_{3}^{2} \\
& \ddot{z}_{p}=\left[a_{2} c_{1} c_{2}+a_{3} c_{1} c_{23}\right] \ddot{\theta}_{1}+\left[-a_{2} s_{1} c_{2}-a_{3} s_{1} c_{23}\right] \dot{\theta}_{1}^{2} \\
& +\left[-2 a_{2} c_{1} s_{2}-2 a_{3} c_{1} s_{23}\right] \dot{\theta}_{1} \dot{\theta}_{2}+\left[-2 a_{3} c_{1} s_{23}\right] \dot{\theta}_{1} \dot{\theta}_{3} \\
& +\left[-a_{2} s_{1} s_{2}-a_{3} s_{1} s_{23}\right] \ddot{\theta}_{2}+\left[-a_{2} s_{1} c_{2}-a_{3} s_{1} c_{23}\right] \dot{\theta}_{2}^{2} \\
& +\left[-2 a_{3} s_{1} c_{23}\right] \dot{\theta}_{2} \dot{\theta}_{3}+\left[-a_{3} s_{1} s_{23}\right] \ddot{\theta}_{3}+\left[-a_{3} s_{1} c_{23}\right] \dot{\theta}_{3}^{2}
\end{aligned}
$$

where

$$
\begin{array}{ll}
c_{1}=\cos \left(\theta_{1}\right) & s_{1}=\sin \left(\theta_{1}\right) \\
c_{2}=\cos \left(\theta_{2}\right) & s_{2}=\sin \left(\theta_{2}\right) \\
c_{3}=\cos \left(\theta_{3}\right) & s_{3}=\sin \left(\theta_{3}\right) \\
c_{23}=\cos \left(\theta_{2}+\theta_{3}\right) & s_{23}=\sin \left(\theta_{2}+\theta_{3}\right)
\end{array}
$$

The expressions for the acceleration of the center of gravity of each link are included in the torque expressions given below. 
Expressions for the torque at each joint of the robot are required because they are explicitly involved in calculating the magnitude of objective functions 1 and 3 . The joint torque expressions take the following form:

$$
\begin{aligned}
& T_{3}=l_{3} m_{3}\left[g c_{23}+a_{2} \dot{\theta}_{2}^{2} S_{3}+a_{2} \ddot{\theta}_{2} c_{3}+l_{3}\left(\ddot{\theta}_{2}+\ddot{\theta}_{3}\right)\right. \\
& \left.+a_{2} \dot{\theta}_{1}^{2} c_{2} s_{23}+l_{3} \dot{\theta}_{1}^{2} c_{23} S_{23}\right]+\left(l_{33}\right)_{3}\left(\ddot{\theta}_{2}+\ddot{\theta}_{3}\right) \\
& +\left\{\left(1_{22}\right)_{3}-\left(1_{11}\right)_{3}\right\}\left(\dot{\theta}_{1}^{2} S_{23} c_{23}\right) \\
& T_{2}=T_{3}+l_{2} m_{2}\left[g C_{2}+l_{2} \ddot{\theta}_{2}+l_{2} \dot{\theta}_{1}^{2} S_{2} C_{2}\right] \\
& +a_{2} m_{3}\left[g c_{2}+a_{2} \ddot{\theta}_{2}+l_{3} c_{3}\left(\ddot{\theta}_{2}+\ddot{\theta}_{3}\right)\right. \\
& -l_{3} S_{3}\left(\dot{\theta}_{2}+\dot{\theta}_{3}\right)^{2}+l_{3} \dot{\theta}_{1}^{2} S_{2} C_{23} \\
& \left.+a_{2} \dot{\theta}_{1}^{2} c_{2} s_{2}\right]+\left(I_{33}\right)_{2} \ddot{\theta}_{2} \\
& +\left\{\left(I_{22}\right)_{2}-\left(I_{11}\right)_{2}\right\} \dot{\theta}_{1}^{2} S_{2} C_{2} \\
& T_{1}=(1,)_{3} S_{23}\left[\ddot{\theta}_{1} S_{23}+2 \dot{\theta}_{1}\left(\dot{\theta}_{2}+\dot{\theta}_{3}\right) C_{23}\right] \\
& +(1,1)_{2}\left[\ddot{\theta}_{1} s_{2}^{2}+2 \dot{\theta}_{1} \dot{\theta}_{2} s_{2} c_{2}\right] \\
& +\left(I_{22}\right)_{3} c_{23}\left[\ddot{\theta}_{1} c_{23}-2 \dot{\theta}_{1}\left(\dot{\theta}_{2}+\dot{\theta}_{3}\right) s_{23}\right] \\
& +\left(I_{22}\right)_{2}\left[\ddot{\theta}_{1} c_{2}^{2}-2 \dot{\theta}_{1} \dot{\theta}_{2} S_{2} c_{2}\right] \\
& -m_{3}\left(l_{3} c_{23}+a_{2} c_{2}\right)\left[2 a_{2} \dot{\theta}_{1} \dot{\theta}_{2} s_{2}\right. \\
& \left.+2 l_{3} \dot{\theta}_{1}\left(\dot{\theta}_{2}+\dot{\theta}_{3}\right) S_{23}-a_{2} \ddot{\theta}_{1} c_{2}-l_{3} \ddot{\theta}_{1} c_{23}\right] \\
& -m_{2} \ell_{2} c_{2}\left[2 l_{2} \dot{\theta}_{1} \dot{\theta}_{2} S_{2}-l_{2} \ddot{\theta}_{1} c_{2}\right]+\left(I_{33}\right)_{1} \ddot{\theta}_{1}
\end{aligned}
$$

\subsection{Determination of an Optimal Motion Program}

The first step in determining an optimal motion program is to determine the optimal traveling time, T. The objective function used is the 
integral over the traveling time of the sum of the square of the joint torques.

$$
I=\int_{0}^{T}\left(T_{1}^{2}+T_{2}^{2}+T_{3}^{2}\right) d t
$$

This objective function has been referred to as objective function 1 in the previous chapter. The same naming will be used here. Note that $T$ will remain variable in order to determine the optimal traveling time. The form of the Rayleigh-Ritz approximating function will be trigonometric with one term added to the $\phi_{0}(t)$ function.

Several starting values of $T$ were used in order to avoid the problem of local minima. Table VIII summarizes the starting points used, the points converged to, and the value of objective function 1 at the points. It can be seen from the table that the optimal time for performing the task is approximately 0.63 seconds. The objective function has a value of $1=15,087$ at this point.

Having determined an optimal traveling time, increasing term trigonometric approximating functions were used to determine how many terms are necessary to approximate the optimal joint motions at $T=0.63$ seconds. Approximations through adding three terms to $\phi_{0}(t)$ were used. Two objective functions were used. These were objective functions 1 and 3 . Objective function 3 performs by minimizing the magnitude of the largest, absolute value of torque occurring at all three joints. Objective function 3 takes the form

$$
\operatorname{minimize} 1=\operatorname{maximum}\left\{\left|T_{1}\right|,\left|T_{2}\right|,\left|T_{3}\right|\right\}
$$

Table IX summarizes the results. 


\section{TABLE VIII}

DETERMINATION OF OPTIMAL TRAVELING TIME FOR THE 3-R ROBOT

\begin{tabular}{|c|c|c|c|c|c|c|}
\hline & arting Poin & & & Converged Poin & & Objective \\
\hline$c_{11}=0.0$ & $\begin{array}{c}c_{21}=0.0 \\
T=0.1\end{array}$ & $c_{31}=0.0$ & $c_{11}=0.1320$ & $\begin{aligned} C_{21} & =0.1110 \\
T & =0.6280\end{aligned}$ & $c_{31}=-0.2680$ & 15,086 \\
\hline$c_{11}=0.0$ & $\begin{array}{l}c_{21}=0.0 \\
T=0.25\end{array}$ & $c_{31}=0.0$ & $c_{11}=0.1003$ & $\begin{aligned} C_{21} & =0.1094 \\
T & =0.6367\end{aligned}$ & $c_{31}=-0.1762$ & 15,881 \\
\hline$c_{11}=0.0$ & $\begin{array}{l}c_{21}=0.0 \\
T=0.5\end{array}$ & $c_{31}=0.0$ & $c_{11}=0.1309$ & $\begin{aligned} c_{21} & =0.1097 \\
T & =0.6274\end{aligned}$ & $c_{31}=-0.2663$ & 15,087 \\
\hline$c_{11}=0.0$ & $\begin{array}{l}c_{21}=0.0 \\
T=1.0\end{array}$ & $c_{31}=0.0$ & $c_{11}=0.0282$ & $\begin{aligned} c_{21} & =0.0935 \\
T & =0.8113\end{aligned}$ & $c_{31}=-0.0639$ & 19,986 \\
\hline$c_{11}=0.0$ & $\begin{array}{l}c_{21}=0.0 \\
T=1.5\end{array}$ & $c_{31}=0.0$ & $c_{11}=0.1752$ & $\begin{aligned} C_{21} & =0.1396 \\
T & =1.209\end{aligned}$ & $c_{31}=-0.8336$ & 16,764 \\
\hline$c_{11}=0.0$ & $\begin{array}{c}C_{21}=0.0 \\
T=2.0\end{array}$ & $c_{31}=0.0$ & $c_{11}=0.2205$ & $\begin{aligned} c_{21} & =0.1923 \\
T & =2.04\end{aligned}$ & $c_{31}=-0.9448$ & 21,698 \\
\hline
\end{tabular}


TABLE IX

COMPARISON OF INCREASING TERM TRIGONOMETRIC APPROXIMATIONS AT $T=0.63$ SECONDS

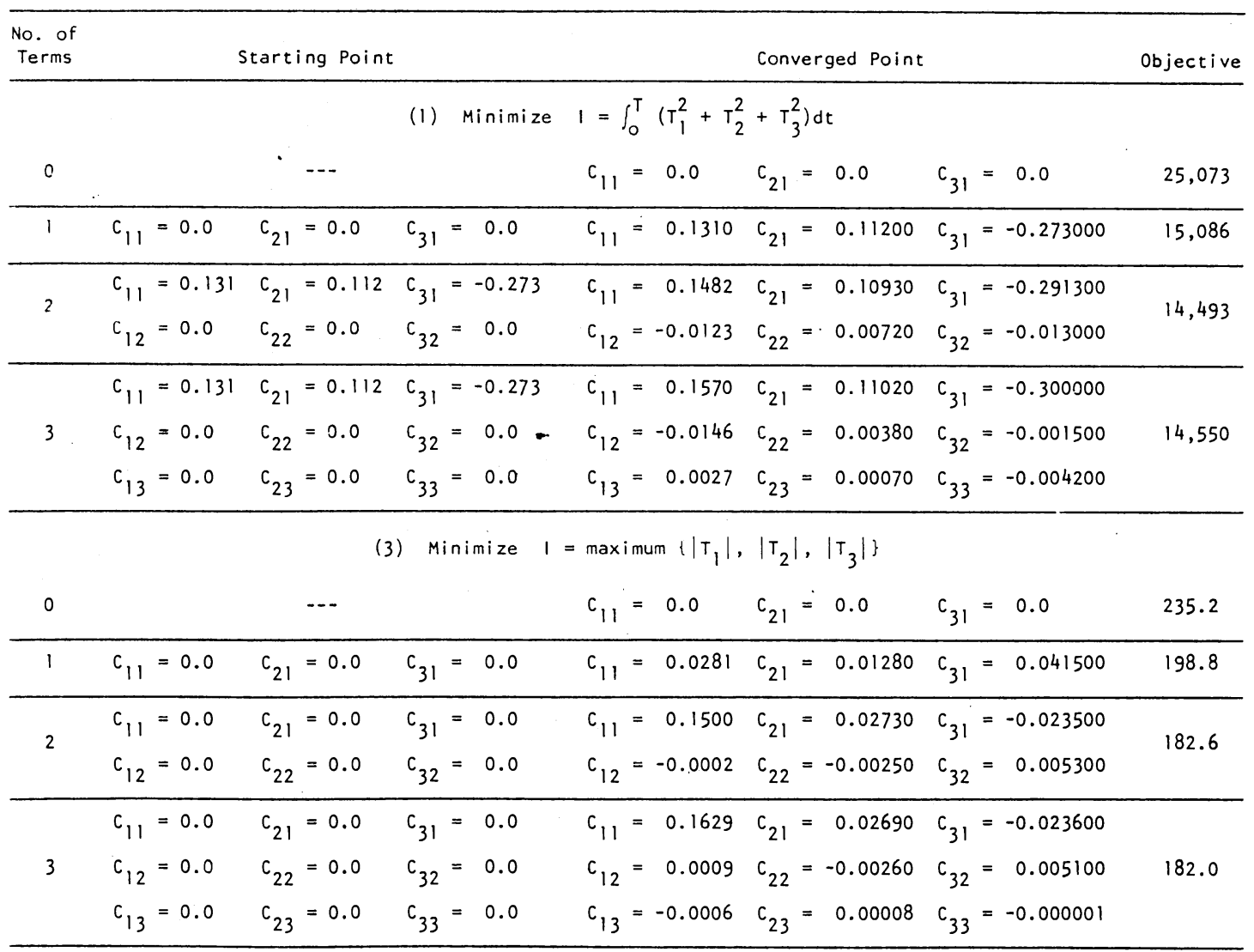


In examining the table for objective function 1 , it can be seen that the value of the objective function continually improves in going from the zero term to the two term approximations. No improvement is obtained by adding three terms to $\phi_{O}(t)$. The objective function actually increases by about $0.4 \%$ over that of the two term solution. This is most likely due to the fact that nine variables are involved, and the program has difficulty in fine tuning all of them to reach the actual minimum. Thus, the optimal motion with respect to objective function 1 is most accurately approximated by adding two terms to the $\phi_{0}(t)$ function.

Similar results were obtained in applying objective function 3 . Considerable improvement is obtained in going from a zero term to a two term solution, but only about $0.4 \%$ improvement in going from a two term to a three term solution. Thus, with respect to objective function 3 , the optimal motion program is most accurately approximated by adding two trigonometric terms to $\phi_{0}(t)$.

A comparison of the weighting coefficients of the two term solutions for objective functions 1 and 3 show that different motion programs are obtained in each case. A comparison must be made between the resulting joint torques for each motion program so that the best solution can be chosen. These comparisons are made in Figures 47 through 49 . Figure 47 compares the torque profiles at joint one. Objective function 1 provides lower peak values of both positive and negative peak torques. The maximum magnitude of torque for objective function 1 is 55 Newton meters. 0bjective function 3 has a maximum magnitude of 130 Newton meters. Figure 48 compares the torques at joint two. The peak values at this joint are nearly identical, with objective function 3 being slightly lower by about 20 Newton meters. Objective function 3 provides a smoother torque profile 


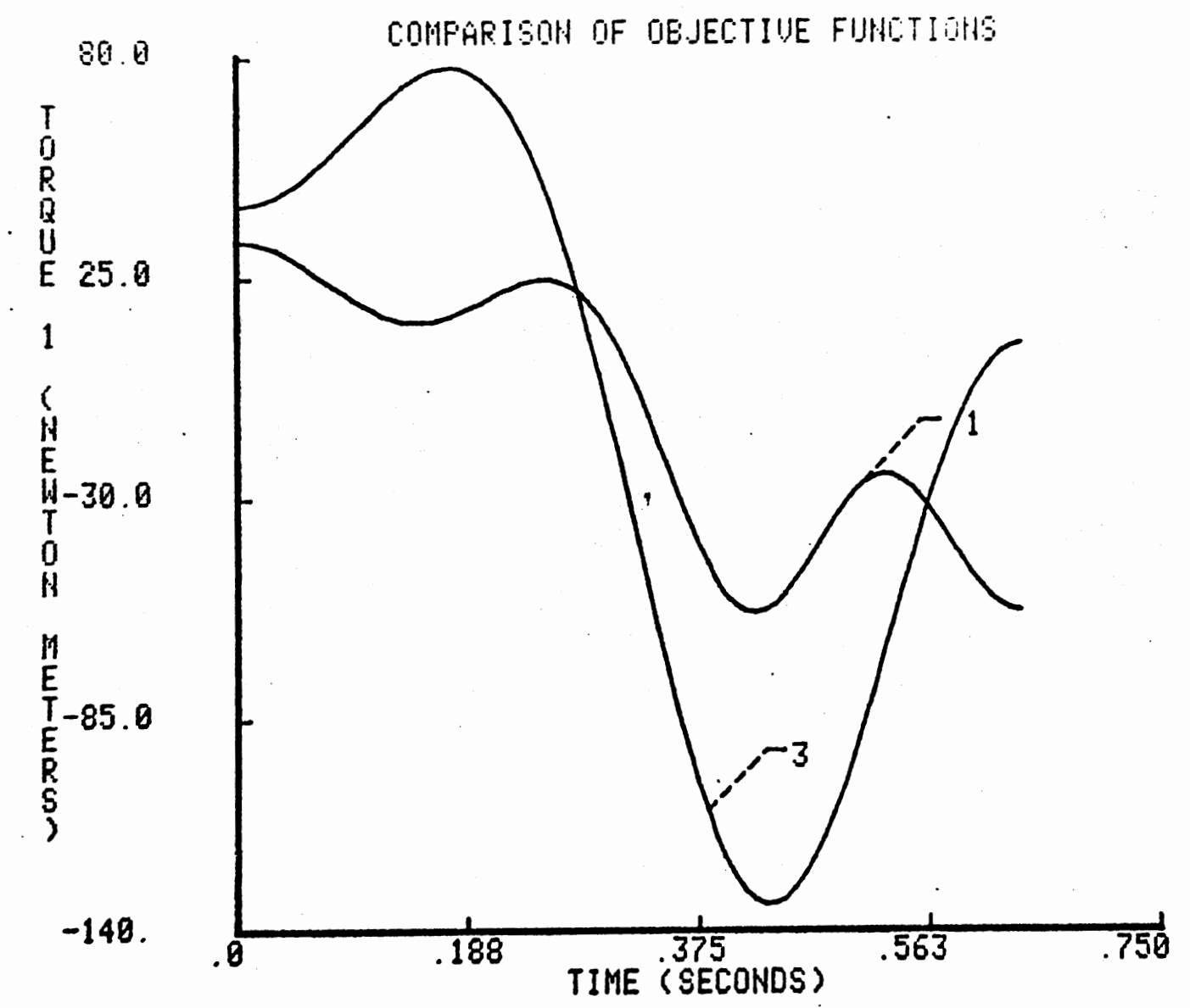

Figure 47. Torques at Joint 1 for Objective Functions 1 and 3 . 


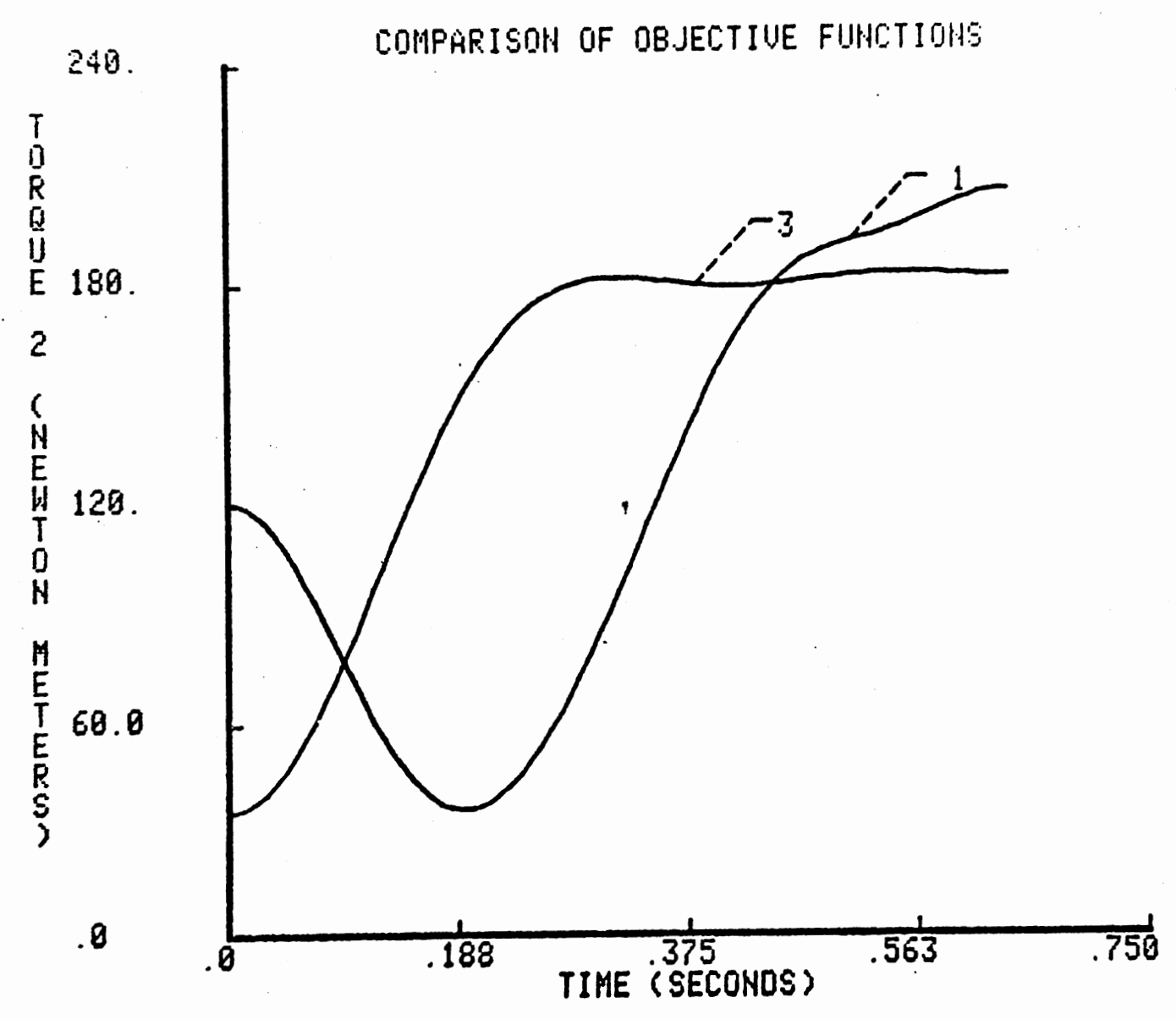

Figure 48. Torques at Joirt 2 for Objective functions 1 and 3 . 


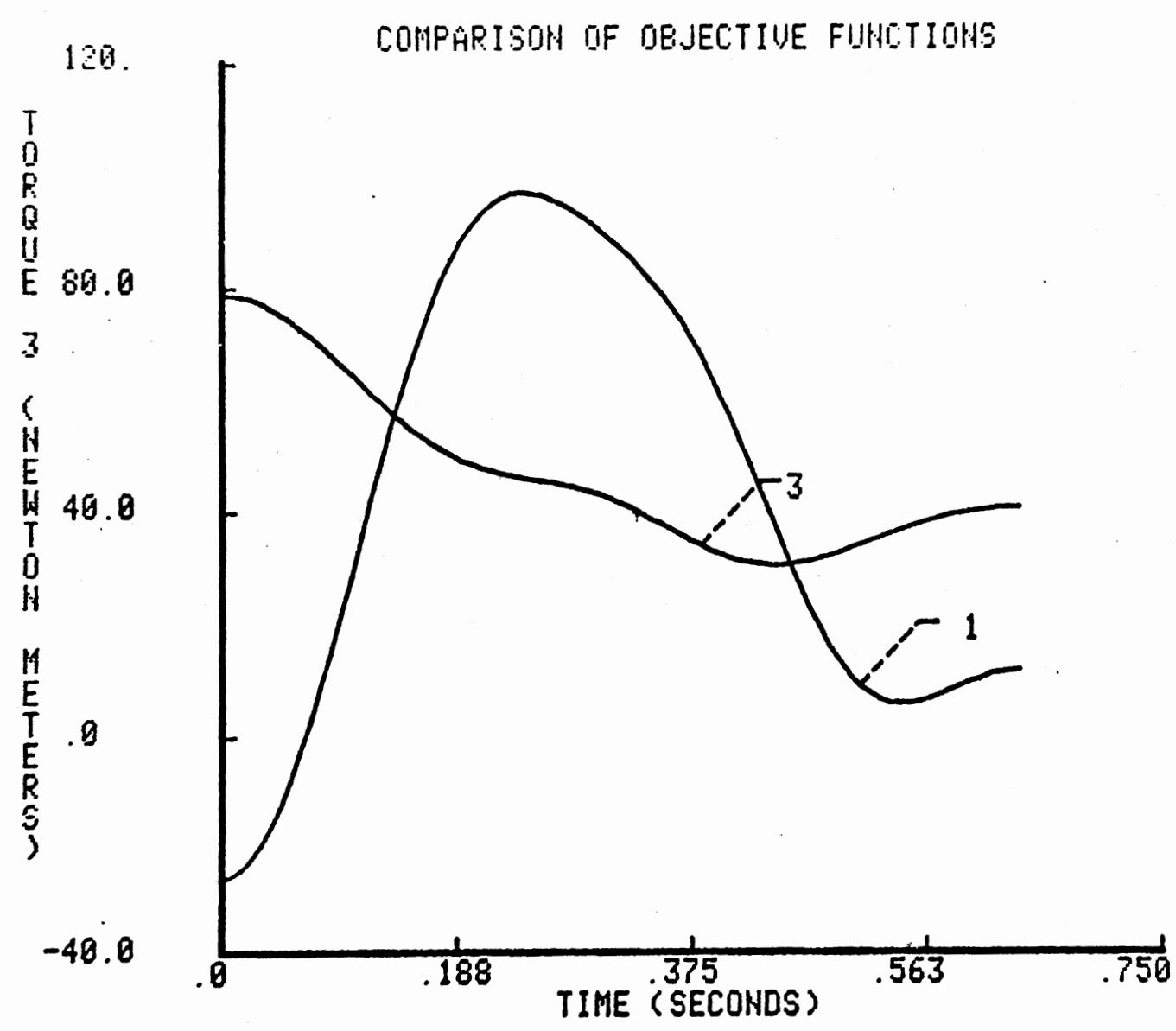

Figure 49. Torques at Joint 3 for objective Functions 1 and 3 . 
at this joint. Figure 49 compares the torques at joint three. Objective function 3 again provides a lower peak torque magnitude by about 20 Newton meters. Also, it provides a smoother torque profile at the joint.

The choice of which solution to use as the optimal motion program is not obvious. Objective function 1 provides a greatly improved torque profile at joint one. However, objective function 3 provides slightly lower values of peak torque, and smoother torque profiles at joints two and three. Before making a choice, the resulting end effector trajectories will be compared. These comparisons are shown in Figures 50 and 51. Figure 50 compares the end effector trajectories in the vertical plane. It can be seen that objective function 1 has an initial, indirect movement during the initial portion of path execution, but overall provides a regular and direct path from $C$ to $D$. Figure 51 compares the end effector paths in the horizontal plane. It can be seen that objective function 1 again provides a more direct path from $C$ to $D$.

The conclusion from these comparisons is that objective function 1 provides a better motion program for the task under consideration. Not only does this motion program provide a direct path from $C$ to $D$, it provides a greatly reduced peak torque magnitude at joint one for a small sacrifice in peak magnitude at joints two and three. Thus, the optimal motion program is approximated by adding two trigonometric terms to the $\phi_{0}(t)$ solution. The weighting coefficients are given in Table $\mid X$.

\subsection{Comparison of Optimal and Standard Solutions}

The results in the last section indicate that an optimal motion program for the $3 R$ robot exists at $T=0.63$ seconds. The optimal motion 


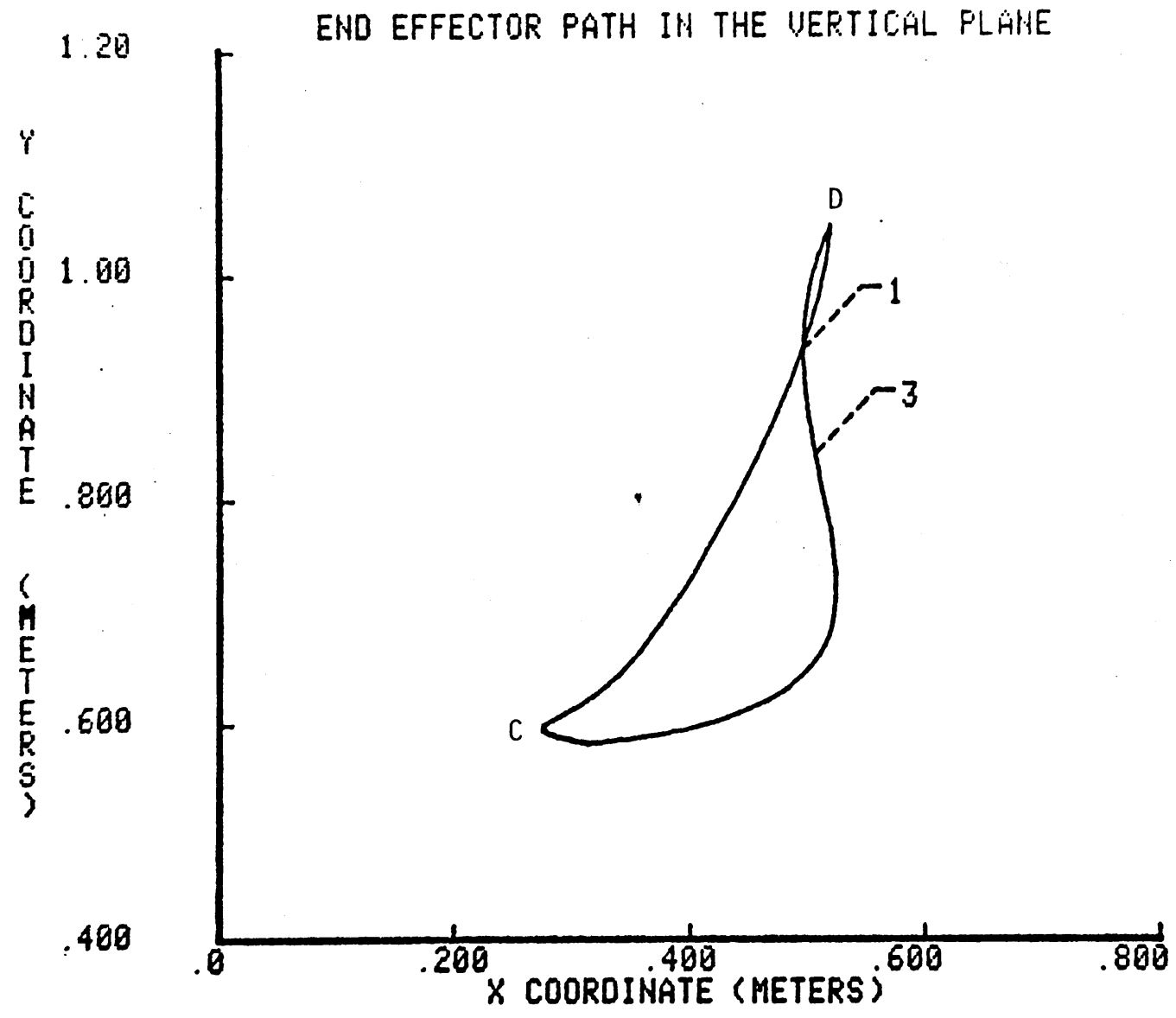

Figure 50. End Effector Paths in Vertical Plane for Objective Functions 1 and 3 . 


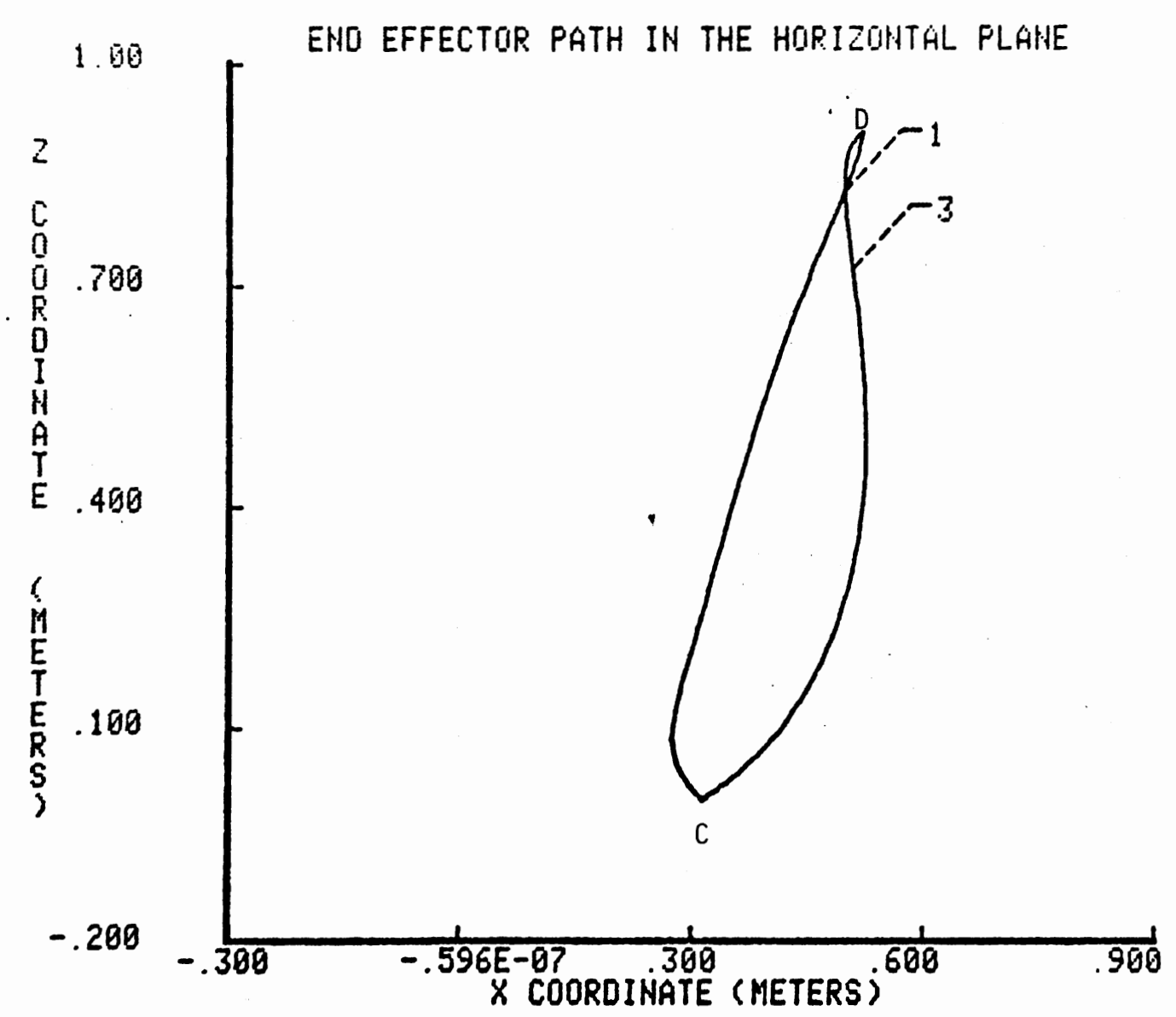

Figure 51. End Effector Paths in Horizontal Plane for Objective Functions 1 and 3 . 
program is approximated by a two term trigonometric approximating function. The resulting weighting coefficients are

$$
\begin{array}{lll}
c_{11}=0.1482 & c_{21}=0.1093 & c_{31}=-0.2913 \\
c_{12}=-0.0123 & c_{22}=0.0072 & c_{32}=-0.0130
\end{array}
$$

The optimal motion program obtained will be compared to the standard solution presented in section 3.7 . This consisted of requiring that the end effector move along a straight $l$ ine path in moving between $C$ and $D$, and the motion along the path would be that of a modified trapezoidal motion program (see Figure 29).

An inverse type problem must be solved in order to obtain the kinematic and dynamic parameters of the robot. The approach used is parallel to that presented for the $2 R$ robot in section 3.7 . The position, velocity and acceleration expressions for the end effector are given in Equations (1) through (9). Note again that three coordinate directions are involved. The Jacobian matrix has dimensions of $3 \times 3$ and takes the following form.

$$
J=\left[\begin{array}{ccc}
\left(-a_{2} s_{1} c_{2}-a_{3} s_{1} c_{23}\right) & \left(-a_{2} c_{1} s_{2}-a_{3} c_{1} s_{23}\right) & \left(-a_{3} c_{1} s_{23}\right) \\
0 & \left(a_{2} c_{2}+a_{3} c_{23}\right) & \left(a_{3} c_{23}\right) \\
\left(-a_{2} c_{1} c_{2}-a_{3} c_{1} c_{23}\right) & \left(a_{2} s_{1} s_{2}+a_{3} s_{1} s_{23}\right) & \left(a_{3} s_{1} s_{23}\right)
\end{array}\right]
$$

The resulting kinematic parameters can be expressed as follows:

$$
\left\{\begin{array}{c}
\Delta \theta_{1} \\
\Delta \theta_{2} \\
\Delta \theta_{3}
\end{array}\right\}=[\mathrm{J}]^{-1}\left\{\begin{array}{c}
\Delta x_{p} \\
\Delta y_{p} \\
\Delta z_{p}
\end{array}\right\}
$$


and

$$
\begin{aligned}
& \left(\theta_{1}\right)_{\text {new }}=\left(\theta_{1}\right)_{\text {old }}+\Delta \theta_{1} \\
& \left(\theta_{2}\right)_{\text {new }}=\left(\theta_{2}\right)_{\text {old }}+\Delta \theta_{2} \\
& \left(\theta_{3}\right)_{\text {new }}=\left(\theta_{3}\right)_{\text {old }}+\Delta \theta_{3}
\end{aligned}
$$$$
\left\{\begin{array}{c}
\dot{\theta}_{1} \\
\dot{\theta}_{2} \\
\dot{\theta}_{3}
\end{array}\right\}=[J]^{-1}\left\{\begin{array}{c}
\dot{x}_{p} \\
\dot{y}_{p} \\
\dot{z}_{p}
\end{array}\right\}
$$

$$
\left\{\begin{array}{c}
\ddot{\theta}_{1} \\
\ddot{\theta}_{2} \\
\ddot{\theta}_{3}
\end{array}\right\}=[\mathrm{J}]^{-1}\left\{\begin{array}{c}
\ddot{x}_{p}^{*} \\
\ddot{y}_{*}^{*} \\
\ddot{z}_{p}^{*}
\end{array}\right\}
$$

where

$$
\begin{aligned}
\ddot{x}_{p}^{*}= & \ddot{x}_{p}+\left(-a_{2} c_{1} c_{2}-a_{3} c_{1} c_{23}\right) \dot{\theta}_{1}^{2}+\left(2 a_{2} s_{1} s_{2}+2 a_{3} s_{1} s_{23}\right) \dot{\theta}_{1} \dot{\theta}_{2} \\
& +\left(-a_{2} c_{1} c_{2}-a_{3} c_{1} c_{23}\right) \dot{\theta}_{2}^{2}+\left(2 a_{3} s_{1} s_{23}\right) \dot{\theta}_{1} \dot{\theta}_{3} \\
& +\left(-2 a_{3} c_{1} c_{23}\right) \dot{\theta}_{2} \dot{\theta}_{3}+\left(-a_{3} c_{1} c_{23}\right) \dot{\theta}_{3}^{2} \\
\ddot{y}_{p}^{*}= & \ddot{y}_{p}+\left(-a_{2} s_{2}-a_{3} s_{23}\right) \dot{\theta}_{2}^{2}+\left(-2 a_{3} s_{23}\right) \dot{\theta}_{2} \dot{\theta}_{3}+\left(-a_{3} s_{23}\right) \dot{\theta}_{3}^{2} \\
\ddot{z}_{p}^{*}= & \ddot{z}_{p}+\left(-a_{2} s_{1} c_{2}-a_{3} s_{1} c_{23}\right) \dot{\theta}_{1}+\left(-2 a_{2} c_{1} s_{2}-2 a_{3} c_{1} s_{23}\right) \dot{\theta}_{1} \dot{\theta}_{2} \\
& +\left(-2 a_{3} c_{1} s_{23}\right) \dot{\theta}_{1} \dot{\theta}_{3}+\left(-a_{2} s_{1} c_{2}-a_{3} s_{1} c_{23}\right) \dot{\theta}_{2} \\
& +\left(-2 a_{3} s_{1} c_{23}\right) \dot{\theta}_{2} \dot{\theta}_{3}+\left(-\dot{a}_{3} s_{1} c_{23}\right) \dot{\theta}_{3}^{2}
\end{aligned}
$$

The torque expressions in Equations (10), (11), and (12) can now be evaluated. 
Figures 52 through 54 compare the joint displacements as a function of time. Figure 52 shows that the two motion programs provide nearly identical motions at joint one. Figure 53 shows that the optimal motion program reduces the overshoot in the motion at joint two. This is due to the fact that the end effector motion is not constrained as it is for the standard solution. Figure 54 shows the displacement at joint three. The optimal and standard solutions have the same profile, but the optimal program provides an initial dwell in the displacement which delays the motion of the joint relative to that of the standard motion program.

Figures 55 through 57 compare the torque profiles at the three robot joints. Figure 55 shows that the magnitude of the peak torque is greatly reduced with the optimal motion program. The standard motion has a peak of about 160 Newton meters, where the optimal solution has a much smaller peak magnitude of torque of about 60 Newton meters. Figure 56 shows a similar reduction in the magnitude of the peak torque. Here a reduction can be seen from 350 Newton meters for the standard motion program, to 200 Newton meters for the optimal motion program. Figure 57 shows that the torque profile for the standard solution is better at the third joint than that of the optimal solution. The optimal solution has a peak magnitude of 100 Newton meters. This is approximately a 40 Newton meter increase over the peak magnitude of the standard solution. However, this peak magnitude increase at joint three does not outweigh the improvements made in the torque profiles at joints one and two. The improvements in the torque profiles is reflected in the value of objective function 1. From Table IX, it was seen that the optimal solution had a value of $1=$ 14,493. The standard solution has a value of $1=54,246$. Thus, the objective function has been reduced by nearly a factor of four. 


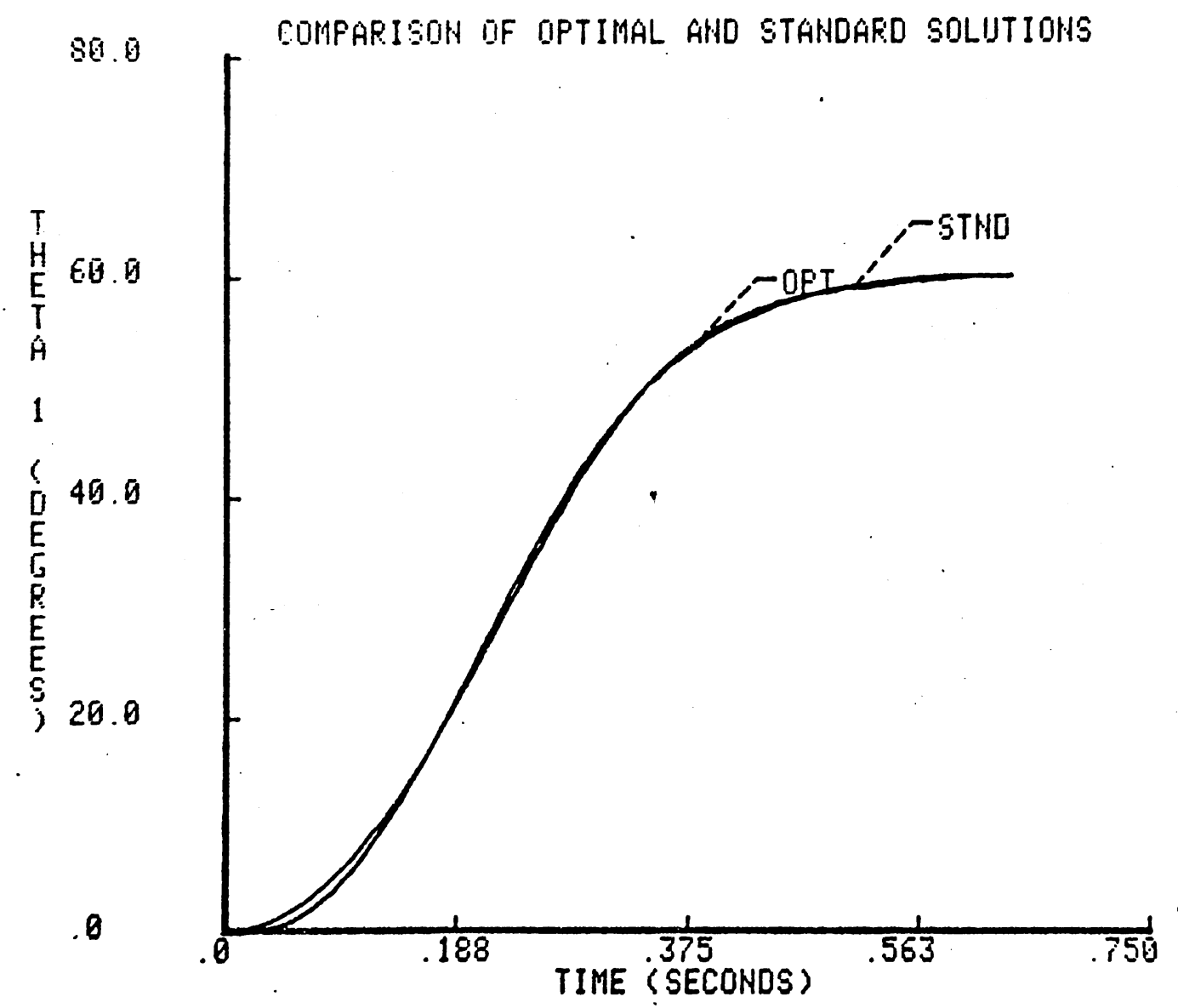

Figure 52. Comparison of Displacements at Joint 1 for Optimal and Standard Solutions. 


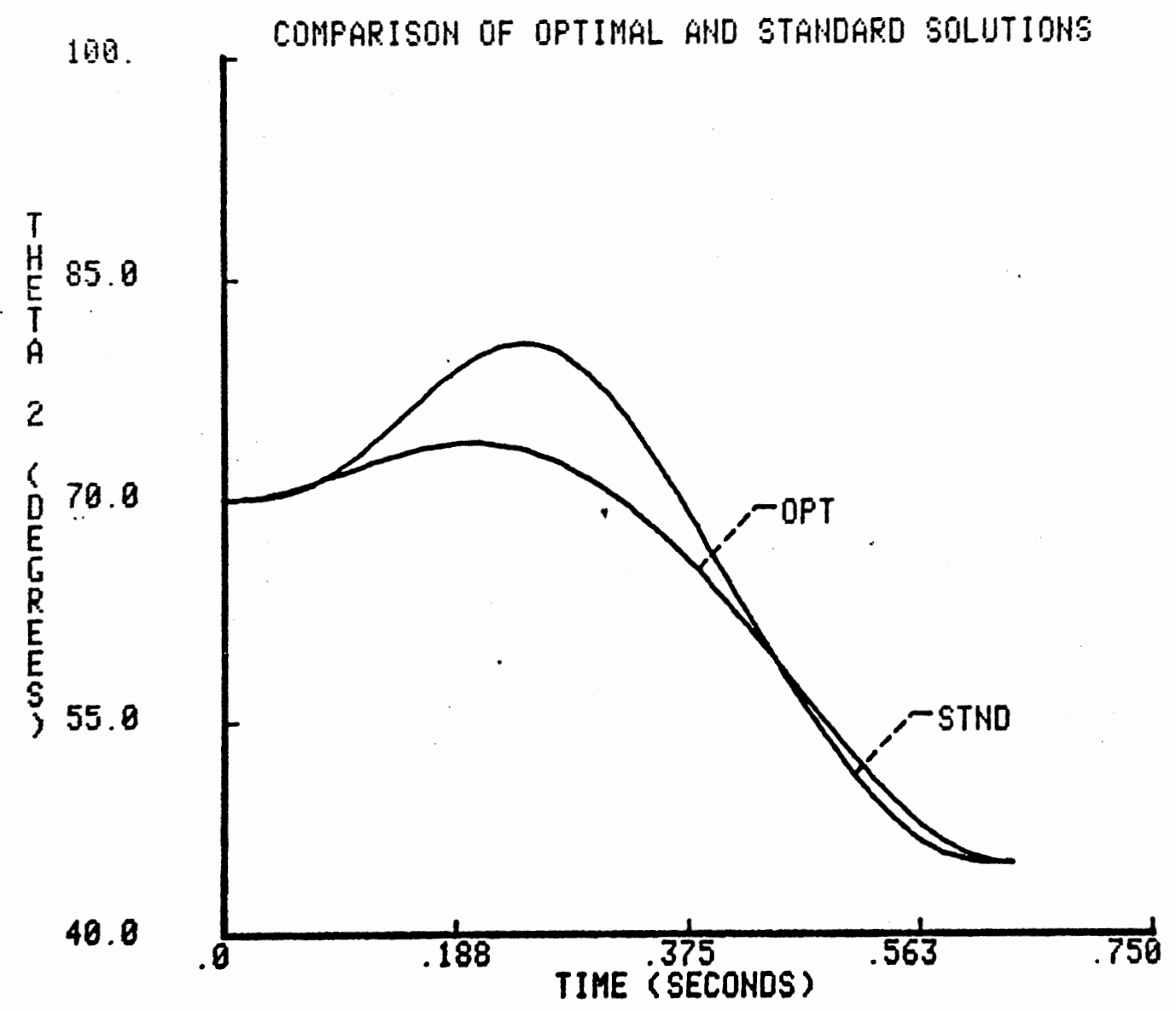

Figure 53. Comparison of Displacements at Joint 2 for Optimal and Standard Solutions. 


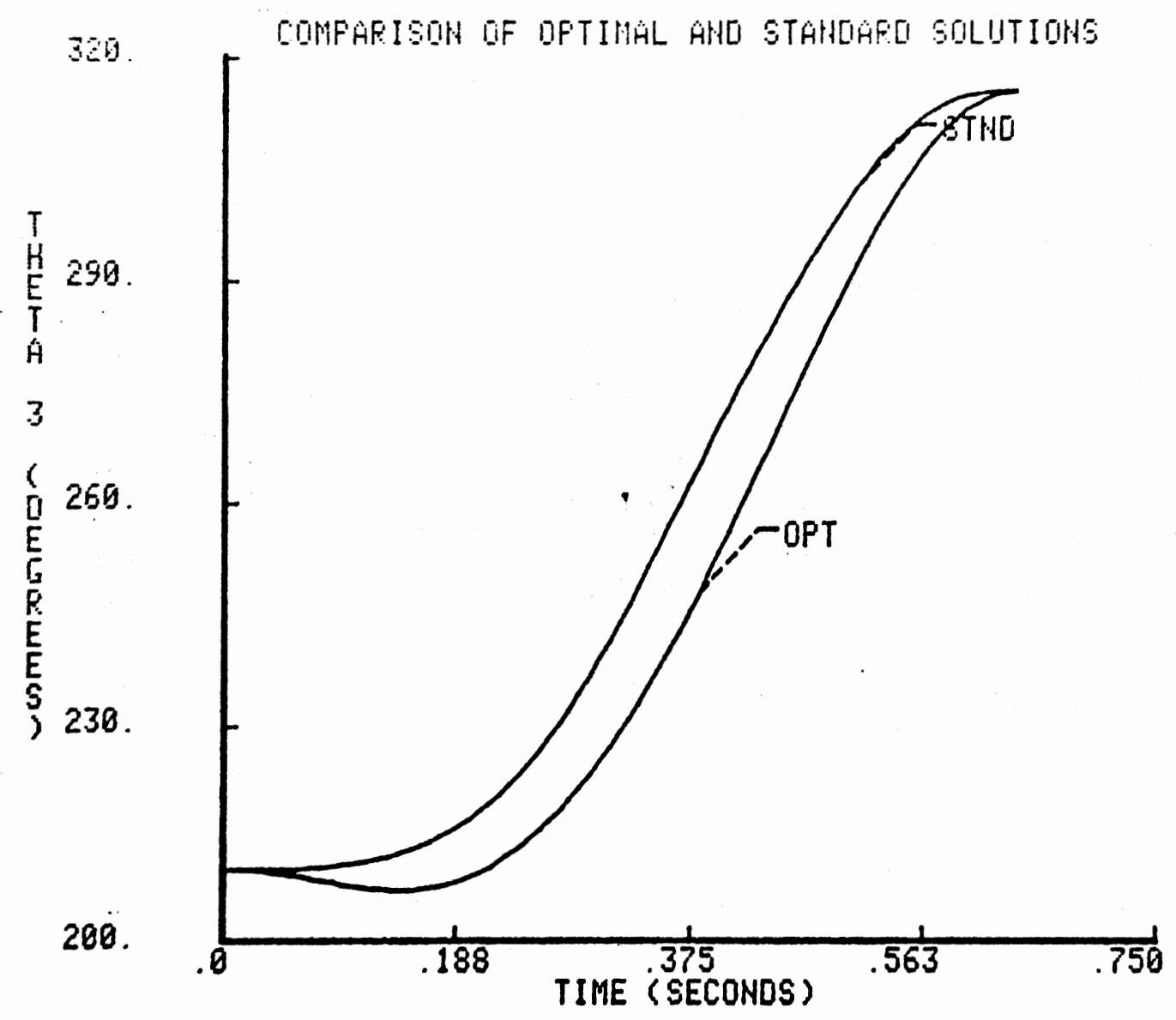

Figure 54. Comparison of Displacements at Joint 3 for Optimal and Standard Solutions. 


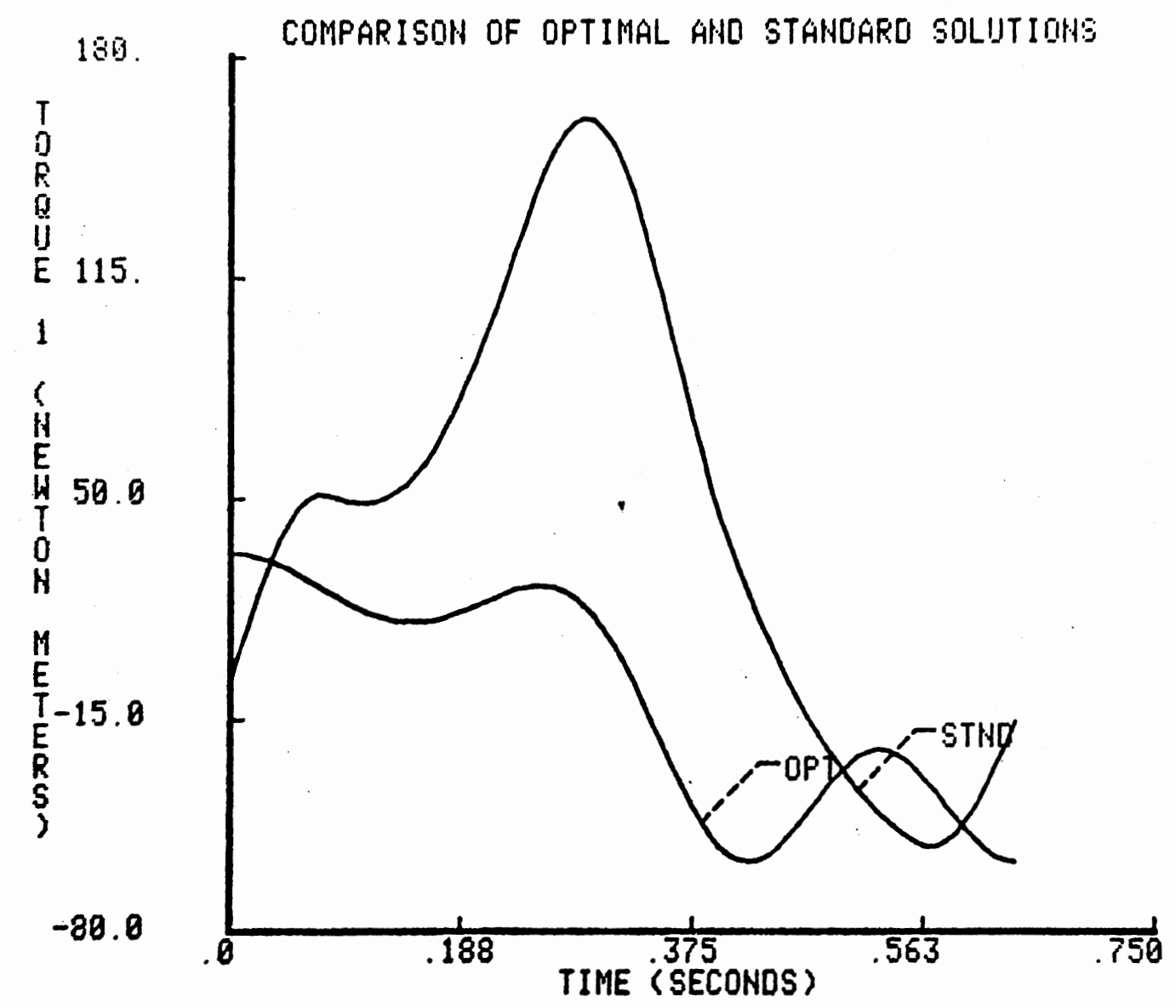

Figure 55. Comparison of Torques at Joint 1 for Optimal and Standard Solutions. 


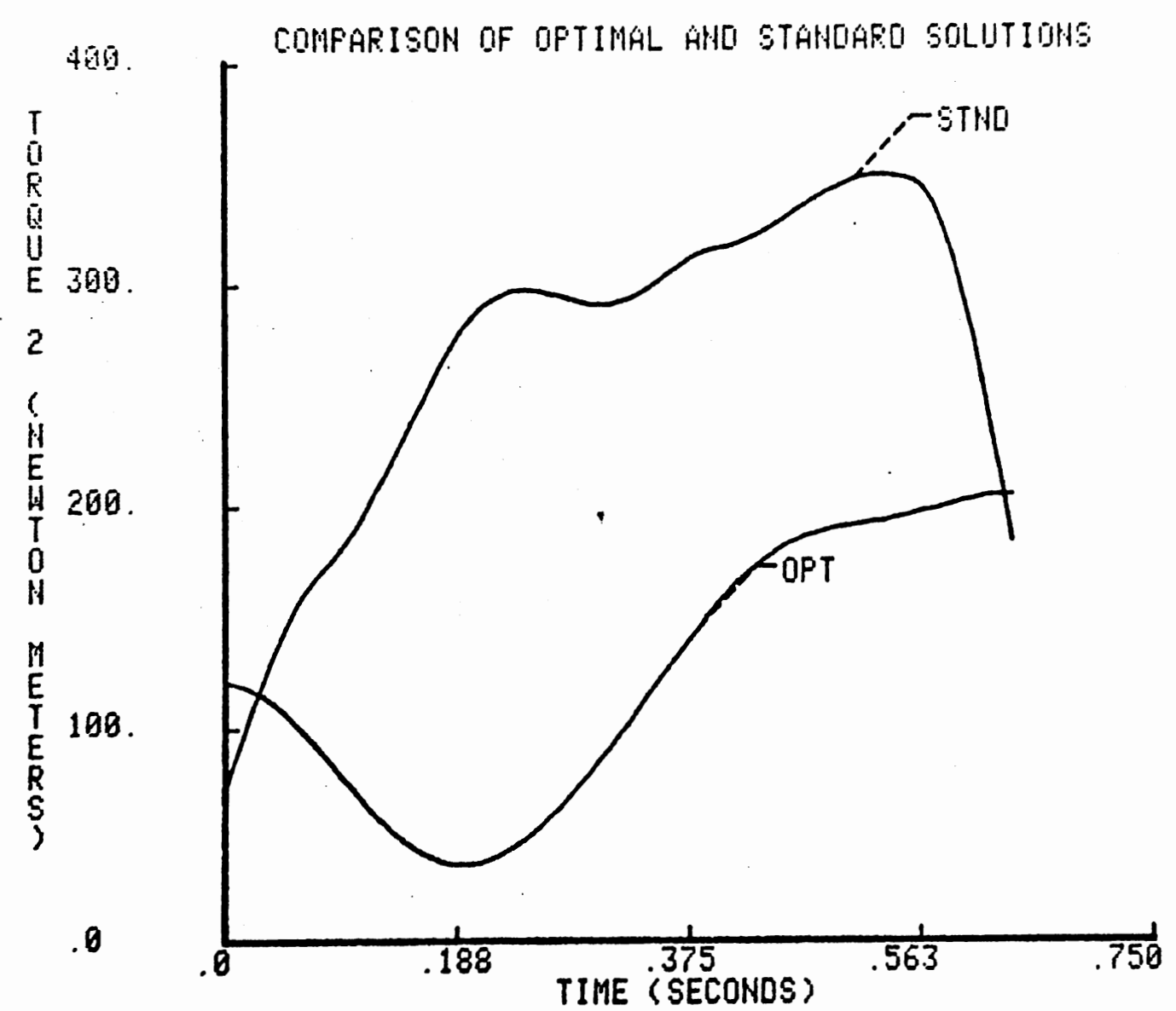

Figure 56. Comparison of Torques at Joint 2 for Optimal and Standard Solutions. 


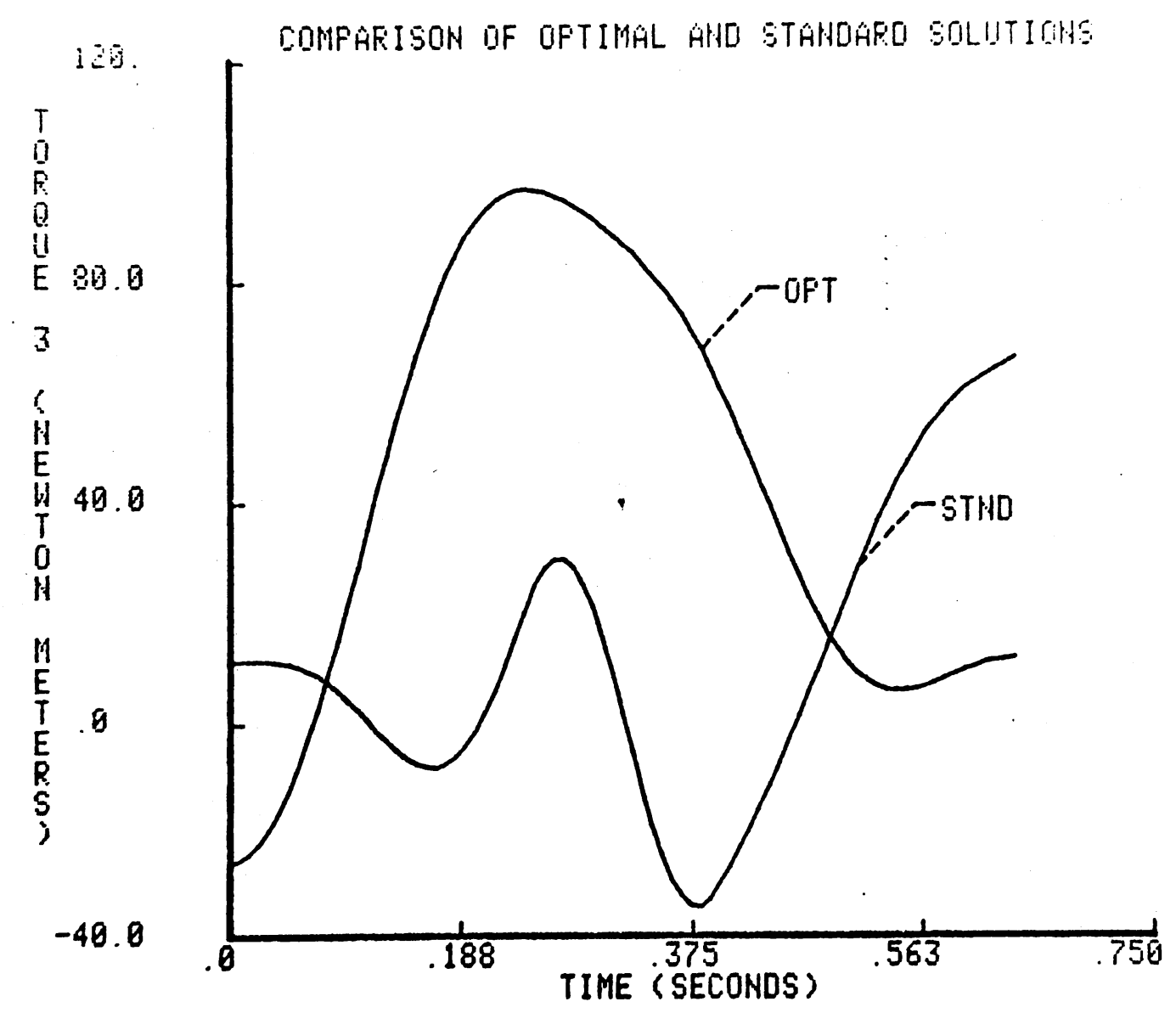

Figure 57. Comparison of Torques at Joint 3 for Optimal and Standard Solutions. 
Figures 58 and 59 compare the end effector trajectories for the optimal and standard solutions. The standard solution follows a straight line path as prescribed. As seen in the previous section, the end effector movement in the vertical plane starts off in a direction away from $D$, but quickly corrects itself and moves on a direct path. The end effector path in the horizontal plane for the optimal motion, as shown in Figure 59, does not deviate significantly from a straight line motion. Thus, not only does the optimal motion program provide improved torque characteristics for the manipulator over that provided by the standard solution, but also provides an acceptable end effector path. 


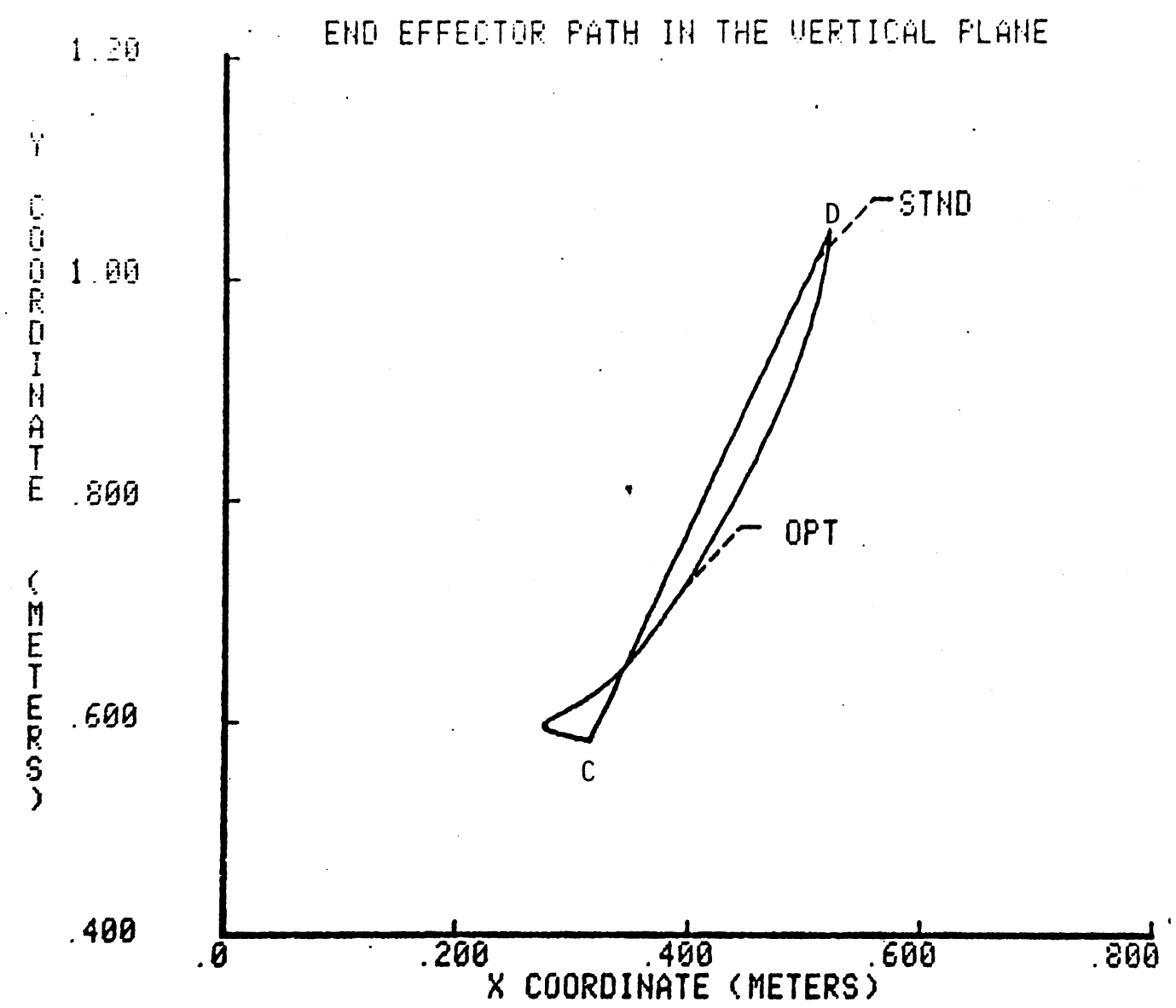

Figure 58. Comparison of End Effector Paths in Vertical Plane for Optimal and Standard Solutions. 


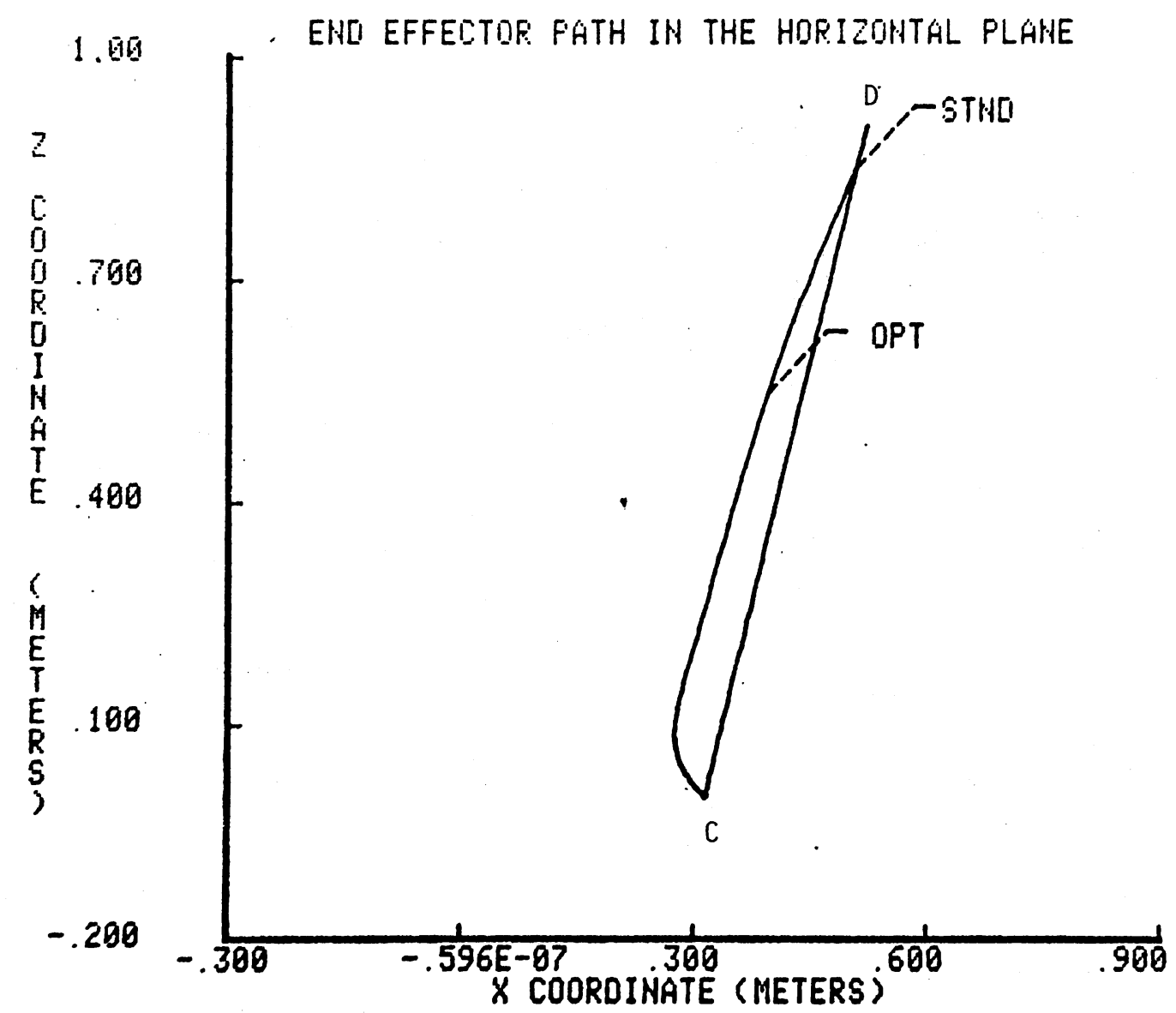

Figure 59. Comparison of End Effector Paths in Horizontal Plane for Optimal and Standard Solutions. 
CHAPTER $V$

SUMMARY AND CONCLUSIONS

\subsection{Summary of Results and Comments}

This thesis has developed and demonstrated a technique to determine optimal motion programs for a robot manipulator to move between two workstations within its workspace. The theory of the technique was developed in Chapter 11 . It has been shown that the optimal motion programming problem can be posed as a calculus of variations problem. The RayleighRitz Method was applied to simplify the formulated problem. The resulting problem can be solved using the SUMT optimization algorithm. The results are the displacement verse time functions for each joint of the robot which cause the robot to execute the task in an optimal fashion.

The technique developed is powerful in that it can handle any robot configuration, consisting of any number of revolute or prismatic pair actuators. The technique was applied to a $2 \mathrm{R}$ robot in Chapter 111 and a $3 R$ robot in Chapter IV. In Chapter III, not only were optimal motion programs determined for the $2 R$ robot, but several factors concerning the solution approach were investigated. The following conclusions were made:

- The number of terms required in the Rayleigh-Ritz approximation should be determined for each problem by using a series of increasing term approximations.

- Polynomial and trigonometric approximating functions perform equally well. 
- Optimal motion programs can be determined at fixed traveling times, or the traveling time can be included as a variable and optimized.

- The choice of objective functions is important. Objective function 1 :

$$
\text { Minimize } 1=\int_{0}^{T}\left(T_{1}^{2}+T_{2}^{2}+\ldots+T_{n}{ }^{2}\right) d t
$$

1. Provides good results in determining the optimal traveling time.

2. May provide the best torque profiles at a fixed traveling time if the fixed traveling time is the optimal time.

Objective function 3 :

$$
\text { Minimize } 1=\operatorname{maximum}\left\{\left|T_{1}\right|,\left|T_{2}\right|, \ldots,\left|T_{n}\right|\right\}
$$

1. May provide best results at a fixed traveling time.

Chapter IV demonstrated that the theory was applicable in three dimensional space. Optimal motion programs were determined for a 3-R robot. The factors determined in the optimal motion programming of the 2-R robot were applied to the $3-R$ problem. Only one difference was noted between the two problems. In applying objective functions 1 and 3 at a fixed value of traveling time, objective function 3 provided equal or superior results to that of object function 1 in the case of the 2-R robot. However, objective function 1 provided superior results for the 3-R robot. This demonstrates the need to apply both objective functions in determining the optimal motion program at a fixed value of traveling time, and compare the results to determine which is superior.

The optimal motion programs determined for the $2-R$ and $3-R$ robots were compared to a standard motion program. They were found to be superior in all cases. Also, an application of the theory was shown in 
Chapter IV. The technique was applied to determine optimal motion programs for different positionings of the task with respect to the robot. This application would allow not only the determination of optimal motion programs, but also the optimal location of the task with respect to the manipulator.

\subsection{Procedure for Solving Optimal Motion Programming Problems}

From the application of the theory to $2-R$ and $3-R$ robots, an approach can be extracted for determining optimal motion programs for a given robot configuration and a prescribed task.

- To determine the optimal traveling time, use the following: One term trigonometric approximating function.

- Minimize $1=\int_{0}^{T}\left(T_{1}{ }^{2}+T_{2}{ }^{2}+\ldots .+T_{n}{ }^{2}\right) d t$

- Multiple starting values of $\mathrm{T}$.

Compare the resulting minima and choose the value of $\mathrm{T}$ corresponding to the smallest value of 1 .

To determine the optimal motion program at a fixed value of $T$ ( $T$ may be optimal or non-optimal), use the following:

- Increasing term trigonometric approximating functions.

- Minimize $1=\int_{0}^{T}\left(T_{1}^{2}+T_{2}^{2}+\ldots .+T_{n}^{2}\right) d t$

and

Minimize $1=\operatorname{maximum}\left\{\left|T_{1}\right|,\left|T_{2}\right|, \ldots .,\left|T_{n}\right|\right\}$

Apply both objective functions using the increasing term approximations. Compare the resulting motion programs based on the torque at each 
joint and the resulting end effector path. Choose the best motion program as the optimal motion program.

\subsection{Extension of the Work}

The present work can be extended in the following ways:

- Provide mathematical models of the actuators so energy consumption can be minimized.

- Provide computerized determination of the dynamic parameters of the robot so that only kinematic data needs to be supplied.

- Apply the theory to higher order robots $(4-R, 5-R, 6-R)$.

- Include prismatic pairs at the robot joints.

The potential also exists for the application of finite element techniques to solve the optimal motion programming problem. Finite elements would be applied in the time domain in order to determine the optimal joint motions in a fashion similar to that applied in this thesis. The advantages of this approach include the fact that the weighting coefficients for the shape functions are the actual joint variables. Also, more variables are included, so greater control over the prescribed values is possible. The disadvantage of this technique is that since more variables are involved, the resulting problem is larger in size and thus more difficult to solve. 


\section{REFERENCES}

[1] Pieper, D.L., and Roth, B., "The Kinematics of Manipulators Under Computer Control." Proceedings of the Ind International Congress on the Theory of Machines and Mechanisms, 2, 159-168 (1969).

[2] Whitney, D.E., "Resolved Motion Rate Control of Manipulators and Human Prostheses." IEEE Transactions on Man-Machine Systems, 10, 47-53 (1969).

[3] Whitney, D.E., "The Mathematics of Coordinated Control of Prosthetic Arms and Manipulators." Transactions of the ASME, Journal of Dynamic Systems, Measurement, and Conirol, 303-309 (1972).

[4] Waldron, K.J., "Geometrically Based Manipulator Rate Control Algorithms." Mechanism and Machine Theory, 17, 379-385 (1982)

[5] Paul, R., "Manipulator Path Control." Transactions of the IEEE, 147-152 (1975).

[6] Paul, R., "The Mathematic's of Computer Controlled Manipulators." Proceedings of the 1977 Joint Automatic Control Conference, 1, 124-131 (1977).

[7] Paul, R., "Manipulator Cartesian Path Control." IEEE Transactions on Systems, Man, and Cybernetics, 9, 702-711 (1979).

[8] Luh, J.Y.S., and Walker, M.W., 'Minimum-Time Along the Path for a Mechanical Arm." Proceedings of the 1977 IEEE Conference on Decision and Control, 1, 755-759 (1977).

[9] Luh, J.Y.S., and Lin, C.S., "Optimum Path Planning for Mechanical Manipulators." Transactions of the ASME, Journal of Dynamic Systems, Measurements, and Control, 102, 142-151 (1980).

$\checkmark[10]$ Taylor, R.H., "Planning and Execution of Straight Line Manipulator Trajectories." IBM Journal of Research and Development, 23, 424-436 (1979).

$\checkmark[11]$ Milenkovic, V., "Computer Synthesis of Continuous Path Robot Motion." Proceedings of the Fifth World Congress on Theory of Machines and Mechanisms, 1332-1335 (1979).

[12] Anderson, T.R. and Paul, R.L., "High Speed Coordinated Control of Industrial Robots." National Science Foundation APR77-14533, 449-461 (1977). 
[13] Luh, J.Y.S., Walker, M.W., and Paul, R.P.C., "Resolved-Acceleration Control of Mechanical Manipulators." IEEE Transactions on Automatic Controls, 25, 468-474 (1980).

[14] Luh, J.Y.S., Walker, M.W., and Paul, R.P.C., "On Line Computational Scheme for Mechanical Manipulators." Transactions of the ASME, Journal of Mechanical Design, 103, 602-607 (1981).

[15] Orlandea, N., and Berenyi, T., "Dynamic Continuous Path Synthesis of Industrial Robots Using ADAMS Computer Program." Transactions of the ASME, Journal of Mechanical Design, $103, \overline{602-607}$ (1981).

[16] Vukobratovic, M., and Kircanski, M., "One Method for Simplified Manipulator Model Construction and its Application in Quazioptimal Trajectory. Synthesis." Mechanism and Machine Theory, 17, 369-378 (1982).

[17] Vukobratovic, M., and Kircanski, M., "A Method for Optimal Synthesis of Manipulation Robot Trajectories." Transactions of the ASME, Journal of Dynamic Systems, Measurements, and Control, 104, 188-193 (1982).

[18] Perez, T.L., "Automatic Planning of Manipulator Transfer Movements." IEEE Transactions on Systems, Man, and Cybernetics, 11, 681$697(1981)$.

Mujtaba, M.S., "Discussign of Trajectory Calculation Methods." Exploratory Study of Computer Integrated Assembly Systems, Stanford University, Artificial Intelligence Laboratory, AIM 285.4 (1977).

[20] Kahn, M.E., and Roth, B., "The Near-Minimum-Time Control of OpenLoop Articulated Kinematic Chains." Transactions of the ASME, Journal of Dynamic Systems, Measurements, and Control, 164-172 (1971).

[21] Cvetkovic, V., and Vukobratovic, M., "Contribution to Controlling Non-Redundant Manipulators." Mechanism and Machine Theory, 16, 81-91 (1981).

[22] Nakamichi, J., and Washizu, K., "Application of the Finite Element Method to an Optimal Control Problem." International Journal for Numerical Methods in Engineering, 12, 1559-1574 (1978).

[23] Nakamichi, J., and Washizu, K., "A Direct Approach Using the Finite Element Method for the Solution of the Linear Optimal Control Problem with a Quadratic Criterion." Journal of The Franklin Institute, 306, 309-319 (1978).

[24] Nakamichi, J., and Washizu, K., "An Application of the Finite Element Technique to an Optimal Trajectory Problem." Proceedings of the Eighth Annual Meeting of Japan Society for Aeronautical and Space Sciences, 118-127 (1978). 
[25] Hildebrand, F.B. Methods of Applied Mathematics. Prentice-Hall Inc., (1965).

[26] Crandall, S.H. Engineering Analysis (A Survey of Numerical Procedures. New York: McGraw-Hill Book Company, Inc., (1956).

[27] Fiacco, A.V., and McCormick; G.P. Nonlinear Programming: Sequential Unconstrained Minimisation Techniques. John Wiley \& Sons, Inc., (1969).

[28] Soni, A.H. Mechanism Synthesis and Analysis. New York: McGraw- . Hill Book Company, (1974).

[29] Brady, M., Hollerbach, J.M., Johnson, T.L., Lozano-Perez, T., and Mason, M.T. Robot Motion: Planning and Control. Cambridge: The MIT Press, (1982).

[30] Hornbeck, R.W. Numerical Methods. New York: Quantum Publishers, Inc., (1975). 
APPENDIX 
C

SUBROUTINE READIN

THIS SUBROUTINE CONTAINS THE INPUT DATA FOR THE ROBOT

AND THE TASK TO BE PERFORMED.

COMMON /SHARE/ $X(100), D E L(100), A(100,100), N, M, M N, N P 1, N M 1$

COMMON /SHARE2/A1, A2,M1, M2,ME, G, I 1, I2 L1 L2, T, EPSINT TINT (10), TFIN $\$(10)$, NTERM, NJOINT

REAL I 1, I 2, M1, M2, ME , L1 , L2, LEQ2

$\mathrm{C}$

C

ROBOT PARAMETERS

NUOINT $=2$

$A 1=.6096$

$M 1=4.536$
$A 2=.6096$

$A 2=.6096$
$M 2=3.175$

$M 2=3 \cdot 175$
$M E=3.175$

$G=9.806$

C

$L 1=A 1 / 2$

$\mathrm{L} 2=\mathrm{A} 2 / 2$

LEQ2 $=(M 2 * L 2+M E * A 2) /(M 2+M E)$

$\mathrm{I} 1=(\mathrm{M} 1 / 12) *(\mathrm{~A} 1 * * 2)$

I $2=(M 2 / 12) *.(A 2 * * 2)+M 2 *(L E Q 2-L 2) * * 2+M E *(A 2-L E Q 2) * * 2$

$\mathrm{L} 2=\mathrm{LEQ2}$

C

$M 2=M 2+M E$

C TASK TO BE PERFORMED

$\operatorname{TINT}(1)=70.0$

$\operatorname{TFIN}(1)=70$.

TINT ( 2) $=245$

$\operatorname{TFIN}(2)=335.0$

C
00000010

00000020

00000030

00000040

00000050

00000060

00000070

c0000080

00000090

00000100

00000110

00000120

00000130

00000130

00000140

00000150

00000160

000170

000180

0000190

00000200

00000210

00000220

00000230

00000240

00000250

00000260

00000270

00000280

00000290

00000300

00000310

00000320

00000330

00000340

00000340

000350

00036

000370

00000380

00000390

00000400

00000410

00000420

00000430

00000440

00000450 
$\operatorname{TINT}(1)=\operatorname{TINT}(1) * \mathrm{CF}$

$\operatorname{TINT}(2)=\operatorname{TINT}(2) * C F$

$\operatorname{TFIN}(1)=\operatorname{TFIN}(1) * C F$

$\operatorname{TFIN}(2)=\operatorname{TFIN}(2) * C F$

C INTEGRATION AND INTERPOLATION PARAMETERS

$T=.6985$

NTERM $=3$

EPSINT $=1 . E-3$

C

RETURN

END

00000460

00000470

$\mathrm{c0000480}$

00000490

00000500

00000510

00000520

00000530

00000540

00000550

00000560

00000570

00000580

00000590 
THIS SUBROUTINE PERFORMS NUMERICAL INTEGRATION USING

A ROMBERG INTEGRATION TECHNIQUE

COMMON /SHARE/ $X(100), D E L(100), A(100,100), N, M, M N, N P 1$, NM 1

COMMON /SHARE2/A1, A2,M1,M2,ME, G, I 1, I2, L1, L2, T, EPSINT, TINT( 10), TF IN

C(10) NTERM, NUOINT

REAL I 1, I 2, M1, M2, ME, L 1 , L2, LEQ2

DIMENSION R( 100,100$)$

IF (I.EQ.O) GO TO 10

VAL $=0.0$

10 TIME $=0$.

TIME $=0.0$

CALL INTERP (TIME, OBJ)

FINT $=O B J$

TIME $=T$

CALL INTERP (TIME, OBJ)

FFIN $=O B J$

$R(1,1)=(T / 2.0) *(F I N T+F F I N)$

$T$ IME $=T / 2.0$

CALL INTERP (TIME, OBJ)

$R(1,2)=R(1,1) / 2.0+(T / 2.0) * O B J$

$R(2,1)=(4 * R(1,2)-R(1,1))$

$J=3$

110 DELTIM $=\mathrm{T} /(2 . * *(\mathrm{~J}-1))$

TIME $=-D E L T I M$

NROM $=2 * * *(J-2)$

SUM $=0.0$

I $I=1$

I I $=1$

IME + $2 * D E L T I M$

CALL INTERP (TIME, OBJ)

$S U M=S U M+O B J$

IF (II.EQ.NROM) GO TO 130

I $I=I I+1$

GO TO 120

$130 \quad R(1, J)=R(1, J-1) / 2 .+D E L T I M * S U M$

00000800

00000810

00000820

00000830

00000840

00000850

00000860

00000870

00000880

00000890

00000900

00000900

00000910

0000920

0000930

0000940

00000950

00000960

00000970

00000980

00000990

00001000

00001010

00001020

00001030 
$K=J+1-L$

$R(L, K)=(4 * *(L-1) * R(L-1, K+1)-R(L-1, K)) /(4 * *(L-1)-1)$

IF (L.EQ.J) GO TO 150

$L=L+1$

GO TO 140

$\operatorname{CONV}=(R(J, 1)-R(J-1,1)) / R(J, 1)$

IF (ABS (CONV). LT.EPSINT) GO TO 16

$J=J+1$

GO TO 110

$\operatorname{VAL}=R(\mathrm{~J}, 1)$

RETURN

00001050

00001060

00001070

00001080

00001090

00001100

00001110

00001120

00001130

00001140

00001150

00001160 
00001220

THIS SUBROUTINE APPROXIMATES THE JOINT MOTIONS USING

00001230

00001240

00001250

00001260

THE DYNAMIC PARAMTERS OF THE ROBOT ARE ALSO EVALUATED

00001270

00001280

00001290

DETERMINED .

COMMON /SHARE/ $X(100)$,DEL $(100), A(100,100), N, M, M N, N P 1, N M 1$

00001300

00001310

00001320

00001330

00001340

COMMON/SHARE2/A1, A2, M1, M2, ME, G, I 1, I2 L1, L2, T, EPSINT, TINT ( 10), TFIN $\$(10)$, NTERM, NUOINT

DIMENSION Z $(10,3)$

$\mathrm{CF}=3.14159 / 180$.

$P I=3,14159$

$\mathrm{C}$
$\mathrm{C}$
$\mathrm{C}$

DO $20 \mathrm{I}=1$, NUOINT

00001360

0001370

00001380

00001390

00001400

00001410

00001420

00001430

00001440

00001450

00001460

00001470

00001480

00001490

00001500

00001500

00001510

00001520

00001530

0001540

0001550

00001560

0001570

00001580

00001590

00001600

00001610 
$\begin{array}{ll}\text { C } & Z(I, 1)-\text { JOINT I ANGULAR DISPLACEMENT } \\ \text { C } & Z(I, 2) \text { - JOINT I ANGULAR VELOCITY }\end{array}$

$Z(I, 3)$ - JOINT I ANGULAR ACCELERATION

00001620

00001630

00001640

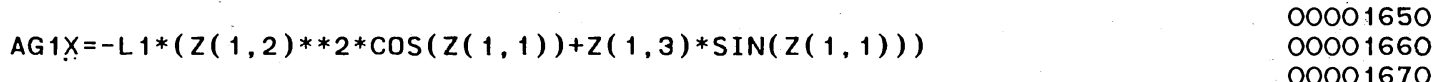

00001670 $\begin{array}{ll}A A=-A 1 *((Z(1,2) * * 2) * \operatorname{CoS}(Z(1,1))+Z(1,3) * \operatorname{SIN}(Z(1,1))) & 00001680 \\ B=-L 2 *(((Z(1,2)+Z(2,2)) * * 2) * \operatorname{COS}(Z(1,1)+Z(2,1))) & 00001690\end{array}$ $B=-L 2 *(((Z(1,2)+Z(2,2)) * * 2) * \operatorname{COS}(Z(1,1)+Z(2,1))) \quad 0001690$ $A G 2 X=A A+B+C$

00001710

00001720

00001730

00001740

00001750

00001750

0001760

001780

0001780

0001790

00001800

00001810

00001820

00001830

00001840

00001850

00001860

00001870

00001880

00001890

00001900

00001910

00001920

00001930

00001940

00001950

0001950

00001970

$O B J=T O R Q 1 * * 2+T O R Q 2 * * 2$

RETURN 


\section{SUBROUTINE GRAD1(I)}

00001980

00001990

00002000

00002010

$\begin{array}{ll}\text { COMMON /SHARE } / X(100), D E L(100), A(100,100), N, M, M N, N P 1, \text { NM1 } & 00002020 \\ \text { IF (I. } 10002030\end{array}$

00002040 DO $20 \mathrm{~K}=1, \mathrm{~N}$

$\operatorname{DEL}(K)=0.0$

20 CONTINUE

RETURN

00002050

00002070

00002080

00002090

00020

CALL DIFF1 (I)

RETURN

00002100

END

00002110

c

SUBROUTINE MATRIX $(J, L)$

00002120

00002130

00002140

00002150

00002160

00002170

COMMON /SHARE/X(100), DEL(100), A (100,100), N, M, MN, NP 1, NM 1 IF (J.EQ.O) GO TO 10

00002180

00002190

RETURN

10 CALL DIFF2(J)

RETURN

00002200

00002210

00002220

00002230 


$$
\begin{gathered}
\text { VITA } \\
\text { Dan Joe Schmitt } \\
\text { Candidate for the Degree of } \\
\text { Master of Science }
\end{gathered}
$$

Thesis: OPTIMAL MOTION PROGRAMMING OF ROBOT MANIPULATORS

Major Field: Mechanical Engineering

Biographical:

Personal Data: Born in Bartlesville, Oklahoma, June 12, 1959, the son of Mr. and Mrs. P. K. Schmitt.

Education: Graduated from Sooner High School, Bartlesville, Oklahoma, in May, 1977; received the Bachelor of Science in Mechanical Engineering degree from Oklahoma State University in 1981; completed requirements for the degree of Master of Science at Oklahoma State University in July, 1983.

Professional Experience: Graduate teaching àssistant, Oklahoma State University, 1981-1983. 